\title{
Progress in the development of exchange-correlation functionals*
}

\author{
Gustavo E. Scuseria and Viktor N. Staroverov \\ Department of Chemistry, Rice University, Houston, Texas 77005, USA
}

March 2, 2006

\begin{abstract}
This review provides a comprehensive account of the recent progress in constructing practical exchange-correlation approximations of Kohn-Sham density functional theory. The emphasis is on the general techniques of density functional design that have been particularly successful in quantum chemistry. Nearly all density functionals embraced nowadays by computational chemists are discussed. Persistent misconceptions about several widely used functionals are clarified.
\end{abstract}

\section{Introduction}

The rise of density functional theory (DFT) to prominence and popularity it enjoys today was hardly anticipated by computational chemists forty or even thirty years ago. As recently as 1983, when DFT was but a footnote in quantum chemistry textbooks, Robert Parr was writing a review "to alert the physical chemistry community to the promise and the charm of the density functional theory of electronic structure of atoms and molecules" [1]. Two decades later, DFT is a household tool for computing everything from atoms to biopolymers. How did this extraordinary reversal of fortunes come about?

Electronic structure methods that use the electron density as the basic variable trace their origin to the Thomas-Fermi [2], Thomas-Fermi-Dirac [3], and related models [4-7] developed in the early years of quantum mechanics. Many similarities with present-day DFT can be also found in Gáspár's exchange potential [8] and Slater's $X \alpha$-methods [9-11]. By the 1960 s, these precursors of DFT were fully developed and used extensively for calculations of atoms and solids, but their impact on molecular quantum chemistry remained insignificant. The Thomas-Fermi and Thomas-Fermi-Dirac models proved to be of little use in chemistry because they can never yield a lower total energy for a molecule than for separated atoms (the "no bonding theorem") [12-14]. The experience of quantum chemists with other DFT prototypes was not so discouraging, but the accuracy of those approximations could not match the accuracy of the increasingly sophisticated wave function techniques.

\footnotetext{
${ }^{*}$ Chapter 24 in: Theory and Applications of Computational Chemistry: The First 40 Years (A Volume of Technical and Historical Perspectives), edited by C. E. Dykstra, G. Frenking, K. S. Kim, and G. E. Scuseria, Elsevier, Amsterdam (2005).
} 
Certain skepticism toward the budding DFT existed also on theoretical grounds. It seemed improbable that a quantitative theory based solely on the electron density could be exact for anything other than model systems like the free-electron gas, simply because the density appears to contain not enough information about electron-electron interactions. The two-electron reduced density matrix was deemed the simplest mathematical object necessary to describe a many-electron system with Coulombic interactions. It was not until 1964 that Hohenberg and Kohn put these doubts to rest by proving their now famous theorem [15]. The Hohenberg-Kohn paper contains two fundamental results: (i) the ground-state electron density uniquely determines the Hamiltonian and, therefore, the ground-state electronic wave function (or a family of degenerate ground-state wave functions [16]) and all properties of the system; (ii) the true density functional for the electronic energy assumes its minimum for the correct ground-state density. These propositions effectively reduce the problem of solving the many-body Schrödinger equation to the problem of minimizing a density functional. This idea can be put to work in various ways, of which the approach of Kohn and Sham [17] has been embraced more often than others.

The challenge of density functional theory consists in the determination or, rather, approximation of the unknown energy density functional. Compared to quantum chemists, solid-state physicists have it easy. The simple formulas derived in the theory of a uniform electron gas work quite well for typical crystals. Not so in chemistry, where the uniform electron gas is not a good approximation for rapidly varying, shell-structured, electron densities. In order to achieve useful accuracy for molecules, even small ones, much more sophisticated approximations are required. The absence of such approximations until the mid-1980s was the single most important reason why DFT conquered chemistry many years after it took a prominent place in solid state physics [18]. Once the first successful density functional approximations for molecules were developed, interest in DFT surged, prompting the discovery of new fundamental results, stimulating the development of scores of density functionals, and generating countless applications. To appreciate this amazing progress, the reader only needs to compare Parr's 1983 review [1] with its follow-up published twelve years later [19]. For more details, we recommend many excellent introductory [20-24] and advanced [25-31] expositions of density functional theory.

To an uninitiated user of quantum chemistry programs, mathematical expressions of density functionals may appear esoteric. The analytic form of many functionals is indeed complicated and non-intuitive, but it often conceals beautifully simple ideas. Lack of familiarity with these ideas and a "black-box" attitude toward the alphabet soup of density functional approximations are in part responsible for the wide-spread sentiment that DFT is effectively an empirical method with no prescription for systematic convergence to the right answer. We hope to convince the reader here that this view is unfair and that the development of density functionals can be, in its own way, a rigorous procedure. We will do so by systematizing and explaining the principal ideas behind modern density functional approximations. Because the most important developments in DFT relevant to chemistry occurred in the last quarter of a century, it is this later period that is primarily covered here.

Although this review focuses on general principles of density functional design, many particular approximations will be discussed in detail. It is not our purpose, however, to present an exhaustive survey of density functionals or to characterize their performance. Such a task would be nearly impossible at a time when the number of published functionals continues to grow at an accelerating pace. Nonetheless, all "popular" exchange-correlation functionals 
for Kohn-Sham DFT (i.e., functionals available in program packages like GAUSSIAN 03 [32] and enjoying wide use) will be covered. Nor shall we touch upon indirectly related subjects such as orbital-free kinetic-energy DFT [33], quantal DFT [34], local-scaling transformation version of DFT [35], and others. Finally, we realize that this review is biased toward our own work and the work of our collaborators and hope that the reader will forgive us any unintended omissions.

\section{Kohn-Sham density functional theory}

Density functional theory aspires to predict exactly properties of many-electron systems without recourse to the wave function, using only the information contained (explicitly or implicitly) in the ground-state electron density. This section reviews the basic DFT formalism and introduces fundamental relations that will recur throughout this work.

\subsection{Motivation for density functional theory}

Consider the problem of solving the nonrelativistic, stationary nucleus, Schrödinger equation

$$
\hat{H} \Psi=E \Psi
$$

involving the $N$-electron Hamiltonian operator (in atomic units)

$$
\hat{H}=-\frac{1}{2} \sum_{i=1}^{N} \nabla_{i}^{2}+\sum_{i=1}^{N} v\left(\mathbf{r}_{i}\right)+\sum_{i<j}^{N} \frac{1}{\left|\mathbf{r}_{i}-\mathbf{r}_{j}\right|}=\hat{T}+\hat{V}+\hat{V}_{\mathrm{ee}}
$$

where $v\left(\mathbf{r}_{i}\right)$ is a multiplicative external potential in which the electrons move. For atoms, molecules, and solids, $v\left(\mathbf{r}_{i}\right)$ is simply the Coulombic potential of the nuclei with charges $Z_{A}$ at positions $\mathbf{R}_{A}$,

$$
v\left(\mathbf{r}_{i}\right)=-\sum_{A}^{\text {nuclei }} \frac{Z_{A}}{\left|\mathbf{r}_{i}-\mathbf{R}_{A}\right|},
$$

although DFT is not restricted to potentials of this form. Multiply Eq. (1) from the left by $\Psi^{*}$ and integrate each term over the spatial $\left(\mathbf{r}_{i}\right)$ and spin $\left(\sigma_{i}\right)$ coordinates not acted upon

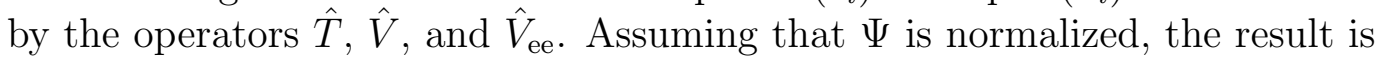

$$
E=-\frac{1}{2} \int\left[\nabla_{\mathbf{r}}^{2} \rho\left(\mathbf{r}, \mathbf{r}^{\prime}\right)\right]_{\mathbf{r}^{\prime}=\mathbf{r}} d \mathbf{r}+\int v(\mathbf{r}) \rho(\mathbf{r}) d \mathbf{r}+\iint \frac{P_{2}\left(\mathbf{r}_{1}, \mathbf{r}_{2}\right)}{\left|\mathbf{r}_{1}-\mathbf{r}_{2}\right|} d \mathbf{r}_{1} d \mathbf{r}_{2},
$$

where we have introduced the one-electron reduced density matrix $\rho\left(\mathbf{r}, \mathbf{r}^{\prime}\right)$ and the pair density

$$
P_{2}\left(\mathbf{r}_{1}, \mathbf{r}_{2}\right)=\frac{N(N-1)}{2} \sum_{\sigma_{1} \ldots \sigma_{N}} \int \ldots \int\left|\Psi\left(\mathbf{r}_{1} \sigma_{1}, \mathbf{r}_{2} \sigma_{2}, \mathbf{r}_{3} \sigma_{3}, \ldots, \mathbf{r}_{N} \sigma_{N}\right)\right|^{2} d \mathbf{r}_{3} \ldots d \mathbf{r}_{N}
$$

The density, reduced density matrix, and the pair density are related by

$$
\rho\left(\mathbf{r}_{1}\right)=\rho\left(\mathbf{r}_{1}, \mathbf{r}_{1}\right)=\frac{2}{N-1} \int P_{2}\left(\mathbf{r}_{1}, \mathbf{r}_{2}\right) d \mathbf{r}_{2} .
$$


The three terms in Eq. (4) represent the kinetic energy of the electrons

$$
T=-\frac{1}{2} \int\left[\nabla_{\mathbf{r}}^{2} \rho\left(\mathbf{r}, \mathbf{r}^{\prime}\right)\right]_{\mathbf{r}^{\prime}=\mathbf{r}} d \mathbf{r},
$$

the electron-nuclear attraction

$$
V=\int v(\mathbf{r}) \rho(\mathbf{r}) d \mathbf{r}
$$

and electron-electron interaction

$$
V_{\mathrm{ee}}=\iint \frac{P_{2}\left(\mathbf{r}_{1}, \mathbf{r}_{2}\right)}{r_{12}} d \mathbf{r}_{1} d \mathbf{r}_{2}
$$

The last term includes the classical Coulomb repulsion and quantum-mechanical exchangecorrelation effects. The separation of the classical and quantum-mechanical parts can be made explicit by writing the pair density as

$$
P_{2}\left(\mathbf{r}_{1}, \mathbf{r}_{2}\right)=\frac{1}{2} \rho\left(\mathbf{r}_{1}\right)\left[\rho\left(\mathbf{r}_{2}\right)+h_{\mathrm{xc}}\left(\mathbf{r}_{1}, \mathbf{r}_{2}\right)\right]
$$

which effectively defines $h_{\mathrm{xc}}\left(\mathbf{r}_{1}, \mathbf{r}_{2}\right)$, the exchange-correlation (xc) hole of an electron at $\mathbf{r}_{1}$. Using Eq. (10) we can rewrite Eq. (9) as

$$
V_{\mathrm{ee}}=J+E_{\mathrm{xc}}^{(\mathrm{c})},
$$

where $J$ is the classical Coulomb repulsion energy

$$
J=\frac{1}{2} \iint \frac{\rho\left(\mathbf{r}_{1}\right) \rho\left(\mathbf{r}_{2}\right)}{r_{12}} d \mathbf{r}_{1} d \mathbf{r}_{2}
$$

and $E_{\mathrm{xc}}^{(\mathrm{c})}$ is the conventional (in wave function-based methods) exchange-correlation energy

$$
E_{\mathrm{xc}}^{(\mathrm{c})}=\frac{1}{2} \iint \frac{\rho\left(\mathbf{r}_{1}\right) h_{\mathrm{xc}}\left(\mathbf{r}_{1}, \mathbf{r}_{2}\right)}{r_{12}} d \mathbf{r}_{1} d \mathbf{r}_{2}
$$

Now Eq. (4) becomes

$$
E=T+V+J+E_{\mathrm{xc}}^{(\mathrm{c})}
$$

Observe that $V$ and $J$ are explicit functionals of $\rho(\mathbf{r})$, but $T$ and $E_{\mathrm{xc}}^{(\mathrm{c})}$ are not. One might suppose that $T$ and $E_{\mathrm{xc}}^{(\mathrm{c})}$ cannot be determined from $\rho(\mathbf{r})$ in principle, since they appear to require the knowledge of the density matrix $\rho\left(\mathbf{r}, \mathbf{r}^{\prime}\right)$ and the pair function $P_{2}\left(\mathbf{r}_{1}, \mathbf{r}_{2}\right)$, respectively. Intuition is wrong here, because the Hohenberg-Kohn theorem [15] asserts that the ground-state energy of a real many-electron system in a static external potential $v(\mathbf{r})$ is a unique functional of the density:

$$
E[\rho]=\int v(\mathbf{r}) \rho(\mathbf{r}) d \mathbf{r}+F[\rho]
$$

where $F[\rho]$ absorbs the kinetic energy and electron-electron interaction terms

$$
F[\rho]=T[\rho]+J[\rho]+E_{\mathrm{xc}}^{(\mathrm{c})}[\rho] .
$$


The Hohenberg-Kohn theorem assures only that the functional $F[\rho]$ exists, but the actual form of $F[\rho]$ is unknown (except for the term $J[\rho]$ ) and must be approximated. Once the number of electrons $N$ is fixed, Hamiltonian operators for any two systems differ only by the external potential $v(\mathbf{r})$. The functional $F[\rho]$ is therefore universal.

The partitioning of $F[\rho]$ into the three components $T, J$, and $E_{\mathrm{xc}}^{(\mathrm{c})}$ in Eq. (16) is by no means unique. Different partitioning schemes give rise to different variants of DFT. In fact, the particular partitioning of Eq. (16), although very natural, is not the one that is normally used. The most popular variant of DFT is the Kohn-Sham formulation, to which we now turn our attention.

\subsection{Kohn-Sham scheme}

The idea of the Kohn-Sham method is best understood as follows. Consider a generalized Hamiltonian of Eq. (2) in which the term $\hat{V}_{\text {ee }}$ is scaled by an electron-electron coupling constant $\lambda$. We are interested in values of $\lambda$ between 0 and 1 . Each value of $\lambda$ corresponds to a distinct universal functional of the density. In Levy's constraint search formulation [36] of the Hohenberg-Kohn principle, this is explicitly stated as

$$
F_{\lambda}[\rho]=\left\langle\Psi_{\rho}^{\min , \lambda}\left|\hat{T}+\lambda \hat{V}_{\mathrm{ee}}\right| \Psi_{\rho}^{\min , \lambda}\right\rangle,
$$

where $\Psi_{\rho}^{\min , \lambda}$ is the $N$-electron wave function that minimizes the expectation value of $\hat{T}+\lambda \hat{V}_{\text {ee }}$ and simultaneously yields the density $\rho(\mathbf{r})$. For real systems, $\lambda=1$, so that $F_{1}[\rho]=F[\rho]$ is the universal functional of interest. This is the problem we wish to solve but cannot.

The value $\lambda=0$ corresponds to a system of noninteracting electrons moving in the external potential $v(\mathbf{r})$. The noninteracting Schrödinger equation is, of course, trivially solvable. The solution is $\Phi_{0}=\Psi_{\rho}^{\mathrm{min}, 0}$, a single Slater determinant of one-electron wave functions (orbitals) $\phi_{i}$ obtained from the single-particle equations

$$
\left[-\frac{1}{2} \nabla^{2}+v(\mathbf{r})\right] \phi_{i}(\mathbf{r})=\epsilon_{i} \phi_{i}(\mathbf{r})
$$

The universal density functional for this noninteracting system is therefore

$$
F_{0}[\rho]=T_{s}[\rho]=-\frac{1}{2} \sum_{i=1}^{N}\left\langle\phi_{i}\left|\nabla^{2}\right| \phi_{i}\right\rangle,
$$

where the density is given by

$$
\rho(\mathbf{r})=\sum_{i=1}^{N}\left|\phi_{i}(\mathbf{r})\right|^{2} .
$$

Note that $T_{s}[\rho]$ is written in terms of orbitals, and so is an implicit functional of $\rho(\mathbf{r})$.

What Kohn and Sham did was to assume that for any real (interacting) system with the ground-state density $\rho(\mathbf{r})$ there always exists a noninteracting system with the same ground-state $\rho(\mathbf{r})$. Then one can rewrite Eq. (16) as

$$
F[\rho]=T_{s}[\rho]+J[\rho]+E_{\mathrm{xc}}[\rho]
$$


where $T_{s}[\rho]$ is the kinetic energy of the noninteracting system computed exactly by Eq. (19), and $E_{\mathrm{xc}}[\rho]$ is the DFT exchange-correlation energy, formally defined as

$$
E_{\mathrm{xc}}[\rho]=T[\rho]-T_{s}[\rho]+E_{\mathrm{xc}}^{(\mathrm{c})}[\rho] .
$$

Application of the variational principle, $\delta E / \delta \rho(\mathbf{r})=0$, to the Kohn-Sham functional

$$
E[\rho]=\int \rho(\mathbf{r}) v(\mathbf{r}) d \mathbf{r}+T_{s}[\rho]+J[\rho]+E_{\mathrm{xc}}[\rho]
$$

subject to the orthonormality constraints $\left\langle\phi_{i} \mid \phi_{j}\right\rangle=\delta_{i j}$, yields $N$ Hartree-type one-electron equations

$$
\left[-\frac{1}{2} \nabla^{2}+v(\mathbf{r})+\int \frac{\rho\left(\mathbf{r}^{\prime}\right)}{\left|\mathbf{r}-\mathbf{r}^{\prime}\right|} d \mathbf{r}^{\prime}+v_{\mathrm{xc}}(\mathbf{r})\right] \phi_{i}(\mathbf{r})=\epsilon_{i} \phi_{i}(\mathbf{r}),
$$

where $\phi_{i}(\mathbf{r})$ are Kohn-Sham orbitals, $\epsilon_{i}$ are Kohn-Sham orbital energies, and $v_{\text {xc }}(\mathbf{r})$ is the exchange-correlation potential

$$
v_{\mathrm{xc}}(\mathbf{r})=\frac{\delta E_{\mathrm{xc}}[\rho]}{\delta \rho(\mathbf{r})},
$$

that is, the functional derivative of $E_{\mathrm{xc}}[\rho]$ with respect to the density. The orbitals $\phi_{i}(\mathbf{r})$ form a Slater determinant $\Phi_{\rho}^{\mathrm{min}}$, called the Kohn-Sham wave function.

Eqs. (20), (24), and (25), known as the Kohn-Sham equations, are formally exact and contain only one unknown term, $E_{\mathrm{xc}}[\rho]$. It is $E_{\mathrm{xc}}$ that is approximated in Kohn-Sham DFT, not the conventional exchange-correlation energy $E_{\mathrm{xc}}^{(\mathrm{c})}$. Exact treatment of the kinetic energy as an orbital-dependent functional is crucial to the practicality of this scheme because $T[\rho]$ and $T_{s}[\rho]$ are notoriously difficult to approximate as explicit functionals of the density $[33,37]$. One can readily show [20] that $T_{s} \leq T$, so that by Eq. (22) $E_{\mathrm{xc}} \geq E_{\mathrm{xc}}^{(\mathrm{c})}$, where the equality holds only for one-electron systems. For a historical account of the developments leading to the announcement of the Hohenberg-Kohn theorem and the formulation of the Kohn-Sham equations, we refer the reader to a memoir of Hohenberg, Kohn, and Sham [38] and Kohn's Nobel lecture [39]. For a modern perspective on DFT fundamentals, we recommend a recent review by Savin, Colonna, and Pollet [40].

\section{Exchange and correlation density functionals}

The problem of finding accurate approximations to $E_{\mathrm{xc}}[\rho]$ is the biggest challenge of KohnSham DFT. The better we understand the exact functional, the better approximations we can design. The following section presents a closer look at the exchange-correlation functional.

\subsection{Exchange-correlation energy}

The formal definition of the exchange-correlation energy by Eq. (22) is not very helpful for designing approximate density functionals. Fortunately, there exist more constructive exact formulas for $E_{\mathrm{xc}}[\rho]$. Observe that by the Hellmann-Feynman theorem [41]

$$
\frac{\partial F_{\lambda}[\rho]}{\partial \lambda}=\left\langle\Psi_{\rho}^{\min , \lambda}\left|\hat{V}_{\mathrm{ee}}\right| \Psi_{\rho}^{\min , \lambda}\right\rangle
$$


where $F_{\lambda}[\rho]$ is given by Eq. (17). Let us integrate Eq. (26) over $\lambda$ from 0 to 1 keeping the density $\rho(\mathbf{r})$ fixed at all $\lambda$. This procedure is called "adiabatic integration" and its result is

$$
\int_{0}^{1} \frac{\partial F_{\lambda}[\rho]}{\partial \lambda} d \lambda=F_{1}[\rho]-F_{0}[\rho]=E_{\mathrm{xc}}[\rho]+J[\rho]
$$

where we have used Eqs. (19) and (21) for $F_{1}[\rho] \equiv F[\rho]$ and $F_{0}[\rho]$, respectively. Combining Eqs. (26) and (27) we obtain the adiabatic connection formula $[42-45]^{1}$

$$
E_{\mathrm{xc}}[\rho]=\int_{0}^{1}\left\langle\Psi_{\rho}^{\min , \lambda}\left|\hat{V}_{\mathrm{ee}}\right| \Psi_{\rho}^{\min , \lambda}\right\rangle d \lambda-J[\rho]=\int_{0}^{1} E_{\mathrm{xc}}^{\lambda}[\rho] d \lambda .
$$

Generalizing the definition of the pair function and exchange-correlation hole in Eq. (10) to intermediate coupling strengths, we now introduce $P_{2}^{\lambda}\left(\mathbf{r}_{1}, \mathbf{r}_{2}\right)$ and $h_{\mathrm{xc}}^{\lambda}\left(\mathbf{r}_{1}, \mathbf{r}_{2}\right)$, and use Eqs. (9)-(11) to rewrite Eq. (28) as

$$
E_{\mathrm{xc}}[\rho]=\frac{1}{2} \int_{0}^{1} d \lambda \iint \frac{\rho\left(\mathbf{r}_{1}\right) h_{\mathrm{xc}}^{\lambda}\left(\mathbf{r}_{1}, \mathbf{r}_{2}\right)}{r_{12}} d \mathbf{r}_{1} d \mathbf{r}_{2} .
$$

In this equation, $\rho\left(\mathbf{r}_{1}\right)$ does not have the subscript $\lambda$ because the electron density is fixed. Since the integration over $\lambda$ is decoupled from the integration over space coordinates, we can define

$$
\bar{h}_{\mathrm{xc}}\left(\mathbf{r}_{1}, \mathbf{r}_{2}\right)=\int_{0}^{1} h_{\mathrm{xc}}^{\lambda}\left(\mathbf{r}_{1}, \mathbf{r}_{2}\right) d \lambda
$$

and rewrite Eq. (29) as

$$
E_{\mathrm{xc}}[\rho]=\frac{1}{2} \iint \rho\left(\mathbf{r}_{1}\right) \frac{\bar{h}_{\mathrm{xc}}\left(\mathbf{r}_{1}, \mathbf{r}_{2}\right)}{r_{12}} d \mathbf{r}_{1} d \mathbf{r}_{2}
$$

Eq. (31) can be regarded as a Kohn-Sham DFT analog of Eq. (13). Let us now make a change of variables $\mathbf{r}=\mathbf{r}_{1}, \mathbf{u}=\mathbf{r}_{2}-\mathbf{r}_{1}$ and integrate over the angular coordinates of $\mathbf{u}$. This gives the spherically-averaged exchange-correlation hole

$$
\bar{h}_{\mathrm{xc}}(\mathbf{r}, u)=\frac{1}{4 \pi} \int_{0}^{2 \pi} d \phi_{u} \int_{0}^{\pi} \bar{h}_{\mathrm{xc}}(\mathbf{r}, \mathbf{r}+\mathbf{u}) \sin \theta_{u} d \theta_{u} .
$$

Using this quantity, Eq. (31) can be written as

$$
E_{\mathrm{xc}}[\rho]=\frac{1}{2} \int \rho(\mathbf{r}) d \mathbf{r} \int_{0}^{\infty} 4 \pi u^{2} \frac{\bar{h}_{\mathrm{xc}}(\mathbf{r}, u)}{u} d u .
$$

This equation serves as the starting point for many density functionals approximations.

In practical Kohn-Sham DFT, the exchange-correlation functional $E_{\mathrm{xc}}$ is usually separated into the exchange and correlation parts,

$$
E_{\mathrm{xc}}[\rho]=E_{\mathrm{x}}[\rho]+E_{\mathrm{c}}[\rho] .
$$

\footnotetext{
${ }^{1}$ The density was held fixed for all $\lambda$ in Refs. [43-45]; it was held fixed only for $\lambda=0$ and 1 in Ref. [42].
} 
The exchange energy is defined by

$$
E_{\mathrm{x}}[\rho]=\left\langle\Phi_{\rho}^{\min }\left|\hat{V}_{\mathrm{ee}}\right| \Phi_{\rho}^{\mathrm{min}}\right\rangle-J[\rho]
$$

where $\Phi_{\rho}^{\mathrm{min}}$ is the Kohn-Sham determinant, while the correlation energy is taken formally as the difference

$$
E_{\mathrm{c}}[\rho]=E_{\mathrm{xc}}[\rho]-E_{\mathrm{x}}[\rho]=\left\langle\Psi_{\rho}^{\min }\left|\hat{V}_{\mathrm{ee}}\right| \Psi_{\rho}^{\min }\right\rangle-\left\langle\Phi_{\rho}^{\min }\left|\hat{V}_{\mathrm{ee}}\right| \Phi_{\rho}^{\min }\right\rangle
$$

where $\Psi_{\rho}^{\mathrm{min}}$ is the exact interacting wave function. The last term in Eq. (36) is nothing but the integral of Eq. (9) with the pair density $P_{2}$ derived from $\Phi_{\rho}^{\min }$. For a single-determinant wave function like $\Phi_{\rho}^{\mathrm{min}}$, the pair density is [46]

$$
P_{2}\left(\mathbf{r}_{1}, \mathbf{r}_{2}\right)=\frac{1}{2} \rho\left(\mathbf{r}_{1}\right) \rho\left(\mathbf{r}_{2}\right)-\frac{1}{2}\left[\rho_{\alpha}\left(\mathbf{r}_{1}, \mathbf{r}_{2}\right) \rho_{\alpha}\left(\mathbf{r}_{2}, \mathbf{r}_{1}\right)+\rho_{\beta}\left(\mathbf{r}_{1}, \mathbf{r}_{2}\right) \rho_{\beta}\left(\mathbf{r}_{2}, \mathbf{r}_{1}\right)\right] .
$$

For spin-compensated systems, substitution of Eq. (37) into Eqs. (9) and (35) yields the following exact expression for the exchange energy

$$
E_{\mathrm{x}}^{\text {exact }}=-\frac{1}{4} \iint \frac{\left|\rho\left(\mathbf{r}_{1}, \mathbf{r}_{2}\right)\right|^{2}}{r_{12}} d \mathbf{r}_{1} d \mathbf{r}_{2}
$$

where $\rho\left(\mathbf{r}_{1}, \mathbf{r}_{2}\right)$ is the Kohn-Sham one-electron density matrix

$$
\rho\left(\mathbf{r}_{1}, \mathbf{r}_{2}\right)=\sum_{i=1}^{N} \phi_{i}\left(\mathbf{r}_{1}\right) \phi_{i}^{*}\left(\mathbf{r}_{2}\right)
$$

Eq. (38) looks exactly like the expression for the exchange energy in the Hartree-Fock (HF) theory, $E_{\mathrm{x}}^{\mathrm{HF}}$. It should be emphasized, however, that $\phi_{i}(\mathbf{r})$ in Eq. (39) are the Kohn-Sham, not Hartree-Fock, orbitals. The two sets of orbitals are different, because they arise from solving different equations. Thus, in general $E_{\mathrm{x}}^{\text {exact }} \neq E_{\mathrm{x}}^{\mathrm{HF}}$. This distinction is important and should always be kept in mind, especially because the terms "exact exchange" and "Hartree-Fock exchange" are often loosely used as synonyms.

The true exchange functional is therefore known exactly. Then why do we need approximate exchange functionals? The problem with Eq. (38) is that it is not an explicit functional of the density, so the corresponding exchange potential cannot be readily evaluated as the functional derivative of $E_{\mathrm{x}}^{\text {exact }}$ with respect to $\rho(\mathbf{r})$. For this purpose, an indirect procedure, called the optimized effective potential (OEP) method [47-55] can be used. Another reason for avoiding the exact exchange functional is that in practice it is very difficult to achieve useful accuracy by combining exact exchange with approximate correlation functionals.

\subsection{Ingredients of density functional approximations}

In principle, the original Kohn-Sham formalism applies to both spin-compensated $\left(\rho_{\alpha}=\rho_{\beta}\right)$ and spin-polarized $\left(\rho_{\alpha} \neq \rho_{\beta}\right)$ systems. In the case of spin-polarized systems, however, the electronic energy is extremely difficult to approximate as a functional of the total density alone. In practice, one always prefers to describe spin-polarized systems using spin-density functional theory. Spin-DFT is a generalization of the original Hohenberg-Kohn principle 
and Kohn-Sham method to systems in the presence of a non-zero external magnetic field. It was first discussed in the original Kohn-Sham paper [17] and elaborated by von Barth and Hedin [56] and by Pant and Rajagopal [57]. In spin-DFT, the basic variables are the spin-up and spin-down electron densities $\rho_{\alpha}(\mathbf{r})$ and $\rho_{\beta}(\mathbf{r})$, and the exchange-correlation energy is a functional of both. In the absence of a magnetic field, spin-DFT gives the same results for spin-polarized systems as the spin-independent DFT, but still operates with functionals of the type $E_{\mathrm{xc}}\left[\rho_{\alpha}, \rho_{\beta}\right]$. This offers a significant practical advantage because approximate spindensity functionals $E_{\mathrm{xc}}\left[\rho_{\alpha}, \rho_{\beta}\right]$ usually provide a much better description of spin-polarized systems than functionals $E_{\mathrm{xc}}[\rho]$.

Let us emphasize again that in spin-DFT the exchange-correlation energy $E_{\mathrm{xc}}$ is a functional of $\rho_{\alpha}(\mathbf{r})$ and $\rho_{\beta}(\mathbf{r})$ alone, and, in principle, these are the only ingredients needed. This appealing picture is gravely complicated by the fact that the dependence of $E_{\mathrm{xc}}$ on $\rho_{\alpha}(\mathbf{r})$ and $\rho_{\beta}(\mathbf{r})$ is highly nonlocal, meaning that: (i) small variations of the densities may cause large variations of the exchange-correlation potential $v_{\mathrm{xc}}$; (ii) $v_{\mathrm{xc}}$ at a given point $\mathbf{r}$ may be very sensitive to changes of the density not only in the vicinity of $\mathbf{r}$, but also at very distant points $\mathbf{r}^{\prime}$. Of course, for practical reasons we want approximate functionals that are explicit and local (or semilocal). To compensate for their locality, such functionals must use some other (local or semilocal) ingredients in addition to the local $\rho_{\sigma}(\mathbf{r})$. These can be derivatives of $\rho_{\sigma}(\mathbf{r})$ (semilocal ingredients) and quantities that are indirectly (nonlocally) determined by the densities. Examples of ingredients of the latter type include Kohn-Sham orbitals and variables constructed from them, provided they are gauge-independent and invariant with respect to unitary transformation of the orbitals.

The most important examples of orbital-dependent ingredients are the Kohn-Sham reduced density matrix of Eq. (39) and the noninteracting kinetic energy density

$$
\tau_{\sigma}(\mathbf{r})=\frac{1}{2} \sum_{k=1}^{\text {occ. }}\left|\nabla \phi_{k \sigma}(\mathbf{r})\right|^{2} .
$$

The name of the latter derives from the fact that $\tau_{\sigma}(\mathbf{r})$ integrates to the Kohn-Sham kinetic energy $T_{s}$ of Eq. (19) for $\sigma$-spin electrons, as can be readily verified via integration by parts. The definition of $\tau_{\sigma}(\mathbf{r})$ by Eq. (40) is gauge-invariant only in the absence of an external magnetic field [58]. Furthermore, it is not unique, because any function that integrates to zero, such as $\nabla^{2} \rho_{\sigma}(\mathbf{r})$, can be added to $\tau_{\sigma}(\mathbf{r})$ without changing the value of $T_{s}$. The noninteracting kinetic energy density is not a far-fetched ingredient as may seem at first sight. It naturally arises in the Taylor series expansion of the Kohn-Sham density matrix (see Section 8.1). Chemical content of the kinetic energy density has been interpreted by Schmider and Becke $[59,60]$. The possibility of constructing functionals of even more complicated variables like $\tau_{2 \sigma}=\frac{1}{4} \sum_{k=1}^{\text {occ. }}\left|\nabla^{2} \phi_{k \sigma}(\mathbf{r})\right|^{2}$, which appear in higher-order density matrix expansions, has also been considered [61].

Another ingredient whose importance is being increasingly recognized [62-67] is the paramagnetic current density, defined in atomic units as

$$
\mathbf{j}_{\sigma}(\mathbf{r})=-\frac{i}{2} \sum_{k=1}^{\text {occ. }}\left[\phi_{k \sigma}^{*}(\mathbf{r}) \nabla \phi_{k \sigma}(\mathbf{r})-\phi_{k \sigma}(\mathbf{r}) \nabla \phi_{k \sigma}^{*}(\mathbf{r})\right] .
$$

This quantity arises in current-dependent DFT, an extension of the Hohenberg-Kohn theory to strong magnetic fields [68-71]. The paramagnetic current density is nonvanishing only for 
degenerate states that are described with complex wave functions (Kohn-Sham determinants in DFT), such as the three configurations of the B atom, where the unpaired electron can occupy either the $2 p_{1}, 2 p_{0}$, or $2 p_{-1}$ orbital. Such states have the same energy but different density distributions. All standard functionals in existence predicts a large artificial separation between the $M_{L}=0$ and $M_{L}= \pm 1$ levels. This unphysical splitting can be drastically reduced by including the current density $\mathbf{j}_{\sigma}(\mathbf{r})$ into approximate functionals [65-67].

Ingredients other than the density do more than just provide additional degrees of freedom for designing density functional approximations. They are necessary if we want an approximation to satisfy exact constraints that are impossible to impose using spin-densities and their derivatives alone. The type of ingredients of $E_{\mathrm{xc}}\left[\rho_{\alpha}, \rho_{\beta}\right]$ forms the basis for Perdew's "Jacob's ladder" classification [72] of density functionals. The lower three rungs of this ladder are, in the ascending order: (i) the local spin-density approximation (LSDA), which employs only $\rho_{\sigma}$; (ii) the generalized gradient approximation (GGA), whose ingredients are $\rho_{\sigma}$ and $\nabla \rho_{\sigma}$; (iii) the meta-GGA, which makes use of $\tau_{\sigma}$ (or $\nabla^{2} \rho_{\sigma}$ ) in addition to the GGA ingredients. The ascent of the ladder consists in embedding increasingly complex ingredients and exact properties in $E_{\mathrm{xc}}\left[\rho_{\alpha}, \rho_{\beta}\right]$ and results, in practice, in better functionals [73].

\subsection{Analytic properties of exchange-correlation functionals}

Although the exact functional $E_{\mathrm{xc}}\left[\rho_{\alpha}, \rho_{\beta}\right]$ remains an enigma, many of its analytic properties, ranging from obvious to subtle, are known [74]. For any possible electron densities, the exchange energy is strictly negative, while the correlation energy is nonpositive:

$$
E_{\mathrm{x}}<0, \quad E_{\mathrm{c}} \leq 0 .
$$

Lieb and Oxford [75] showed that the exchange-correlation energy of electrons in Coulombic systems is bounded also from below:

$$
E_{\mathrm{x}}\left[\rho_{\alpha}, \rho_{\beta}\right] \geq E_{\mathrm{xc}}\left[\rho_{\alpha}, \rho_{\beta}\right] \geq C_{\mathrm{LO}} \int \rho^{4 / 3}(\mathbf{r}) d \mathbf{r},
$$

where $-1.44 \geq C_{\mathrm{LO}} \geq-1.68[76]$.

For one-electron densities $\rho_{1}(\mathbf{r}), E_{\mathrm{x}}$ must cancel the spurious Coulomb self-repulsion energy:

$$
E_{\mathrm{x}}\left[\rho_{1}, 0\right]+J\left[\rho_{1}\right]=0,
$$

while $E_{\mathrm{c}}$ must vanish altogether:

$$
E_{\mathrm{c}}\left[\rho_{1}, 0\right]=0 .
$$

Eqs. (44) and (45) were stressed in the self-interaction correction scheme of Perdew and Zunger [77].

For uniform electron densities, $E_{\mathrm{xc}}\left[\rho_{\alpha}, \rho_{\beta}\right]$ should reduce to the formulas for the exchangecorrelation energy of a uniform free-electron gas (the LSDA):

$$
E_{\mathrm{xc}}\left[\rho_{\alpha}, \rho_{\beta}\right]=E_{\mathrm{xc}}^{\mathrm{LSDA}}\left[\rho_{\alpha}, \rho_{\beta}\right], \quad \text { if } \quad \rho_{\sigma}(\mathbf{r})=\text { const. }
$$

A large number of known exact properties of density functionals involve coordinate scaling transformations of the density. Most of such relations have been derived by Levy and coworkers [78-84]. The uniform scaling of the density is defined by

$$
\rho_{\gamma}(\mathbf{r})=\gamma^{3} \rho(\gamma \mathbf{r})
$$


The effect of this transformation is to contract or thin the density while preserving its normalization. Coordinate scaling constraints for exchange and correlation functionals are reviewed by Levy [85]. The most important among these constraints are the following:

$$
\begin{gathered}
E_{\mathrm{x}}\left[\rho_{\gamma}\right]=\gamma E_{\mathrm{x}}[\rho], \\
\lim _{\gamma \rightarrow \infty} E_{\mathrm{c}}\left[\rho_{\gamma}\right]>-\infty .
\end{gathered}
$$

The exchange functionals appropriate to spin-compensated and to spin-polarized systems are related to each other by the spin-scaling relation [86]

$$
E_{\mathrm{x}}\left[\rho_{\alpha}, \rho_{\beta}\right]=\frac{1}{2}\left(E_{\mathrm{x}}\left[2 \rho_{\alpha}\right]+E_{\mathrm{x}}\left[2 \rho_{\beta}\right]\right),
$$

where $E_{\mathrm{x}}[\rho] \equiv E_{\mathrm{x}}[\rho / 2, \rho / 2]$. The spin-scaling relation effectively defines the resolution of the exchange energy into two parallel-spin contributions, $E_{\mathrm{x}}^{\sigma \sigma}=\frac{1}{2} E_{\mathrm{x}}\left[2 \rho_{\sigma}\right]$, where $\sigma=\alpha, \beta$. Note that $E_{\mathrm{x}}$ has no opposite-spin components. Eq. (50) is respected by all exchange energy approximations in existence. In the literature, exchange functionals are often stated in the form $E_{\mathrm{x}}[\rho]$ only, while the form $E_{\mathrm{x}}\left[\rho_{\alpha}, \rho_{\beta}\right]$ (the one actually used) is implied through Eq. (50). For the correlation energy, there is no simple spin-scaling relation similar to Eq. (50).

\section{Strategies for designing density functionals}

DFT would be the ultimate practical theory if one could derive the exact exchange-correlation functional in a closed form, as were the Thomas-Fermi kinetic energy and Dirac exchange energy functionals for the uniform electron gas. This appears to be a hopeless task. DFT would still be almost perfect if, by analogy with wave function methods, we had a mechanical prescription for the systematic improvement of approximations that guarantees their convergence to the right answer. In principle, Görling-Levy perturbation theory [87-89] and "ab initio DFT" [90-92] offer such prescriptions, but these approaches abandon the idea of a universal density functional and, for all practical purposes, are hardly different from wave function techniques. In practice, one has to devise density functional approximations relying on many little bits of information about the true functional and compensating for lack of mechanical recipes with insight and ingenuity. As the success of DFT attests, this is a workable plan. We distinguish the following six approaches to designing density functional approximations.

1. Local density approximations (LDA). This group includes functionals derived in the analytic theory of a uniform electron gas and applied, directly or with empirical modifications, to non-uniform densities. All LDA functionals have the form

$$
E_{\mathrm{xc}}^{\mathrm{LDA}}[\rho]=\int e_{\mathrm{xc}}(\rho) d \mathbf{r}
$$

where the exchange-correlation energy density $e_{\mathrm{xc}}(\rho)$ is a function of $\rho(\mathbf{r})$ only.

2. Density-gradient expansion (DGE). These are formal analogs of the three-dimensional Taylor expansion of the exchange-correlation energy in derivatives of the density:

$$
E_{\mathrm{xc}}^{\mathrm{DGE}}[\rho]=\int\left[e_{\mathrm{xc}}^{(0)}(\rho)+e_{\mathrm{xc}}^{(1)}(\rho) \nabla \rho+e_{\mathrm{xc}}^{(2)}(\rho)|\nabla \rho|^{2}+\ldots\right] d \mathbf{r} .
$$


Derivation of the coefficients $e_{\mathrm{xc}}^{(k)}(\rho)$ is very involved mathematically, and the performance of such nonempirical functionals is reasonable only for slowly-varying densities.

3. Constraint satisfaction. A more successful approach consists in designing functionals of the form

$$
E_{\mathrm{xc}}[\rho]=\int e_{\mathrm{xc}}\left(\rho, \nabla \rho, \nabla^{2} \rho, \tau, \ldots\right) d \mathbf{r}
$$

where the integrand is constructed to satisfy chosen exact constraints. The constraints in question may concern the asymptotic behavior of $e_{\mathrm{xc}}$ and $v_{\mathrm{xc}}$, upper and lower bounds on the energy, density scaling transformations, and other properties of the true functional. A number of approximations of this type are fully nonempirical.

4. Modeling the exchange-correlation hole. Functionals of this type are based on Eqs. (31) or (38) and form one of the largest and most diverse groups. For example, the exchange hole may be approximated by a truncated Taylor series expansion in $\rho$, $\nabla \rho, \nabla^{2} \rho$, and $\tau$. The correlation hole may be derived from an approximate correlated wave function or modeled after the correlation hole of an analytically solvable problem. The general analytic form of these functionals is the same as in Eq. (53). Functionals based on an exchange-correlation hole model may be fully nonempirical or contain fitted parameters.

5. Empirical fits. Functionals of this type are designed by fitting reasonably chosen analytic forms of $E_{\mathrm{xc}}[\rho]$ to experimental values of thermochemical and/or other properties of atoms and molecules. The analytic form may be borrowed directly from functionals of any other group or simply postulated without a rigorous derivation. Some fitted ("optimized") functionals are linear combinations of pre-existing functionals. The general form of such approximations is

$$
E_{\mathrm{xc}}[\rho]=\sum_{k} C_{k} \int e_{\mathrm{xc}}^{(k)}\left(\rho, \nabla \rho, \nabla^{2} \rho, \tau, \ldots ; a_{k}, b_{k}, \ldots\right) d \mathbf{r}
$$

where $C_{k}, a_{k}, b_{k}, \ldots$ are adjustable parameters.

6. Mixing exact and approximate exchange. These functionals, termed hybrids, have the form

$$
E_{\mathrm{xc}}^{\mathrm{hybrid}}[\rho]=\int\left[a e_{\mathrm{x}}^{\text {exact }}(\mathbf{r})+b e_{\mathrm{x}}^{\mathrm{DFT}}(\mathbf{r})+e_{\mathrm{c}}^{\mathrm{DFT}}(\mathbf{r})\right] d \mathbf{r}
$$

where the mixing coefficients $a$ and $b$ may be constants or depend on $\mathbf{r}$. In the latter case, functionals of this form are called "local hybrids" [93].

The majority of density functional approximations in current use fall into one of these categories. The scheme presented above, however, is neither perfect nor complete. For example, the LDA for exchange may be viewed as a functional derived from the exact exchange hole $h_{\mathrm{x}}^{\mathrm{LDA}}(\mathbf{r}, u)$ of a uniform electron gas [it is given by Eq. (57) below] and placed in group 4. Functionals of any group may have adjustable parameters, not just optimized functionals. Exact constraints are often explicitly imposed on density functionals based on an exchange-correlation hole model, not only on functionals of group 3. The above scheme does not include several interesting but less common methods such as the weighted-density 
approximation [94-97], the phase-space approach [98, 99], Laurent series expansions in terms of homogeneous functionals [100,101] or moments of the density [102], and many others. Nevertheless, we believe that the classification of density functionals by the method of their derivation is instructive. In what follows, we will use it as a map to navigate the realm of approximate density functionals.

\section{$5 \quad$ Local density approximations}

In a narrow sense, the local density approximation consists in applying the exact results of the theory of a uniform electron gas to real nonuniform densities. More generally, an LDA is any approximation of the form

$$
E_{\mathrm{xc}}^{\mathrm{LDA}}[\rho]=\int \rho(\mathbf{r}) \epsilon_{\mathrm{xc}}(\rho) d \mathbf{r},
$$

where $\epsilon_{\mathrm{xc}}(\rho)=\epsilon_{\mathrm{x}}(\rho)+\epsilon_{\mathrm{c}}(\rho)$ is the exchange-correlation energy per particle of the electron gas, which is a function of the density only.

\subsection{Local density approximation for exchange}

The exchange energy of a uniform electron gas can be evaluated analytically by the method of Bloch [103] or Dirac [3]. Details of both approaches are discussed by Gombás [4], Bethe [104], Slater [105], and Parr and Yang [20]. The outline of the derivation is as follows. The KohnSham orbitals for a uniform electron gas are plane waves, $\phi_{\mathbf{k}}(\mathbf{r})=V^{-1 / 2} e^{i \mathbf{k} \cdot \mathbf{r}}$, where $V$ is the volume of the box. Given the orbitals, one calculates the Kohn-Sham density matrix of Eq. (39) by replacing the sum over occupied orbitals $\mathbf{k}$ with an integral over a sphere of radius $k_{F}=\left(3 \pi^{2} \rho\right)^{1 / 3}$. Then a transformation to relative coordinates $\mathbf{r}=\mathbf{r}_{1}, \mathbf{u}=\mathbf{r}_{2}-\mathbf{r}_{1}$ and angle-averaging yield the LDA exchange hole

$$
h_{\mathrm{x}}^{\mathrm{LDA}}(\mathbf{r}, u)=-\frac{9}{2} \rho(\mathbf{r})\left[\frac{\sin \left(k_{F} u\right)-k_{F} u \cos \left(k_{F} u\right)}{\left(k_{F} u\right)^{3}}\right]^{2},
$$

which determines the exchange energy by Eq. (33). The final result is

$$
E_{\mathrm{x}}^{\mathrm{LDA}}=-C_{\mathrm{x}} \int \rho^{4 / 3}(\mathbf{r}) d \mathbf{r}, \quad \text { where } \quad C_{\mathrm{x}}=\frac{3}{4}\left(\frac{3}{\pi}\right)^{1 / 3} .
$$

Equivalently, the LDA exchange energy per particle is

$$
\epsilon_{\mathrm{x}}^{\mathrm{LDA}}(\rho)=-C_{\mathrm{x}} \rho^{1 / 3}=-\frac{3}{4}\left(\frac{3}{2 \pi}\right)^{2 / 3} \frac{1}{r_{s}},
$$

where $r_{s}=(3 / 4 \pi \rho)^{1 / 3}$ is the radius of a sphere that contains the charge of one electron. Eq. (59) was first obtained by Wigner and Seitz [106].

The LDA exchange formula (58) is exact for a uniform electron gas but underestimates the exchange energy of inhomogeneous systems. Generally, LDA is more accurate than the Hartree-Fock method but falls far short of chemical accuracy $(1 \mathrm{kcal} / \mathrm{mol})$. 
The extension of Eq. (58) to spin-polarized systems is called the local spin-density approximation (LSDA). According to Eq. (50), the LSDA exchange energy is

$$
E_{\mathrm{x}}^{\mathrm{LSDA}}\left[\rho_{\alpha}, \rho_{\beta}\right]=-2^{1 / 3} C_{\mathrm{x}} \int\left(\rho_{\alpha}^{4 / 3}+\rho_{\beta}^{4 / 3}\right) d \mathbf{r}
$$

Although Eq. (60) is all one needs for practical purposes, let us cast it in a different form (for reasons that will become clear when we discuss the LSDA for correlation). Introducing the relative spin-polarization

$$
\zeta=\frac{\rho_{\alpha}-\rho_{\beta}}{\rho_{\alpha}+\rho_{\beta}}
$$

and using $\rho_{\alpha}=\frac{1}{2}(1+\zeta) \rho$ and $\rho_{\beta}=\frac{1}{2}(1-\zeta) \rho$, we rewrite Eq. (60) as

$$
E_{\mathrm{x}}^{\mathrm{LSDA}}\left[\rho_{\alpha}, \rho_{\beta}\right]=\int \rho \epsilon_{\mathrm{x}}(\rho, \zeta) d \mathbf{r}
$$

where

$$
\epsilon_{\mathrm{x}}(\rho, \zeta)=-\frac{1}{2} C_{\mathrm{x}} \rho^{1 / 3}\left[(1+\zeta)^{4 / 3}+(1-\zeta)^{4 / 3}\right] .
$$

For a spin-compensated ("paramagnetic", $\zeta=0$ ) electron gas,

$$
\epsilon_{\mathrm{x}}=\epsilon_{\mathrm{x}}^{P}=-C_{\mathrm{x}} \rho^{1 / 3},
$$

and for fully-polarized ("ferromagnetic", $\zeta= \pm 1$ )

$$
\epsilon_{\mathrm{x}}=\epsilon_{\mathrm{x}}^{F}=-2^{1 / 3} C_{\mathrm{x}} \rho^{1 / 3} .
$$

For intermediate spin-polarizations $0<\zeta<1$, one can write $\epsilon_{\mathrm{x}}(\rho, \zeta)$ as an exact interpolation between the para- and ferromagnetic cases,

$$
\epsilon_{\mathrm{x}}(\rho, \zeta)=\epsilon_{\mathrm{x}}^{P}(\rho)+\left[\epsilon_{\mathrm{x}}^{F}(\rho)-\epsilon_{\mathrm{x}}^{P}(\rho)\right] f(\zeta),
$$

where the interpolating function is readily shown to be

$$
f(\zeta)=\frac{1}{2} \frac{\left[(1+\zeta)^{4 / 3}+(1-\zeta)^{4 / 3}-2\right]}{\left(2^{1 / 3}-1\right)} .
$$

Ernzerhof and Scuseria [107] suggested approximating $E_{\mathrm{x}}$ using the LDA functional in which the actual $\rho(\mathbf{r})$ is replaced by a fictitious density $\tilde{\rho}(\mathbf{r})$ defined by $\tau(\mathbf{r})=C_{F} \tilde{\rho}^{5 / 3}(\mathbf{r})$, where $\tau(\mathbf{r})$ is the kinetic energy density of the actual nonuniform system. This leads to the local $\tau$-approximation (LTA)

$$
E_{\mathrm{x}}^{\mathrm{LTA}}[\tau]=\frac{C_{\mathrm{x}}}{C_{F}^{4 / 5}} \int \tau^{4 / 5}(\mathbf{r}) d \mathbf{r}
$$

where $C_{F}=\frac{10}{3}\left(3 \pi^{2}\right)^{2 / 3}$. Eq. (68) can be viewed as an alternative to the conventional LDA. In numerical tests, the LTA was found to be "complementary" to the LDA. It predicts more accurately exchange energy contributions to the atomization energies in those cases where LDA is in large error, and vice versa. For a uniform electron gas, LDA is equivalent to LTA. 


\subsection{Local density approximation for correlation}

Correlation is a much more difficult problem than exchange, so exact analytic forms of $\epsilon_{\mathrm{c}}^{\mathrm{LDA}}(\rho)$ are known only for two limiting cases. The first is the high-density (weak correlation) limit of a spin-compensated uniform electron gas

$$
\epsilon_{\mathrm{c}}^{P}\left(r_{s}\right)=A_{\mathrm{GB}} \ln r_{s}+B+r_{s}\left(C \ln r_{s}+D\right), \quad r_{s} \ll 1 .
$$

The constants $A_{\mathrm{GB}}$ and $B$ were evaluated by Gell-Mann and Brueckner [108], $C$ and $D$ by Carr and Maradudin [109]. Specifically, in hartree units $\left(E_{h}\right)$,

$$
A_{\mathrm{GB}}=\frac{1-\ln 2}{\pi^{2}} \approx 0.031091
$$

The second case is the low-density (strong correlation) limit obtained by Nozières and Pines [110] and Carr [111]

$$
\epsilon_{\mathrm{c}}^{P}\left(r_{s}\right)=\frac{1}{2}\left(\frac{U_{0}}{r_{s}}+\frac{U_{1}}{r_{s}^{3 / 2}}+\frac{U_{2}}{r_{s}^{2}}+\ldots\right), \quad r_{s} \gg 1,
$$

where $U_{k}$ are again known constants. Similar formulas exist for $\epsilon_{\mathrm{c}}^{F}\left(r_{s}\right)$.

The exact numerical values of $\epsilon_{\mathrm{c}}^{P}\left(r_{s}\right)$ and $\epsilon_{\mathrm{c}}^{F}\left(r_{s}\right)$ are known, with small statistical uncertainties, for several intermediate values of $r_{s}$ from Monte Carlo simulations of the uniform electron gas carried out by Ceperley and Alder [112]. Based on these results, several interpolation formulas for $\epsilon_{\mathrm{c}}^{P}\left(r_{s}\right)$ and $\epsilon_{\mathrm{c}}^{F}\left(r_{s}\right)$ have been devised to connect the high- and low-density limits [Eqs. (69) and (71)] and simultaneously reproduce the Ceperley-Alder data for intermediate $r_{s}$. Three such parametrizations are widely used in quantum-chemical codes.

Perdew and Zunger [77] (PZ81) suggested the following parametrization of the CeperleyAlder data for the spin-compensated and spin-polarized cases

$$
\epsilon_{\mathrm{c}}^{\mathrm{PZ} 81, i}\left(r_{s}\right)= \begin{cases}\frac{\gamma}{1+\beta_{1} r_{s}^{1 / 2}+\beta_{2} r_{s}}, & \text { if } r_{s} \geq 1, \\ A \ln r_{s}+B+C r_{s} \ln r_{s}+D r_{s}, & \text { if } r_{s}<1\end{cases}
$$

where $\gamma, \beta_{1}, \beta_{2}, A, B, C$, and $D$ are parameters, different for $i=P$ and $i=F$. In particular, $A^{P}=2 A^{F}=A_{\mathrm{GB}}$. The PZ81 parametrization has several shortcomings, such as an artificial discontinuity of second and higher derivatives at $r_{s}=1$.

Vosko, Wilk, and Nusair [113] (VWN) proposed a more accurate but less transparent representation

$$
\begin{aligned}
\epsilon_{\mathrm{c}}^{\mathrm{VWN}, i}(x)= & A\left\{\ln \frac{x^{2}}{X(x)}+\frac{2 b}{Q} \tan ^{-1} \frac{Q}{2 x+b}\right. \\
& \left.-\frac{b x_{0}}{X\left(x_{0}\right)}\left[\ln \frac{\left(x-x_{0}\right)^{2}}{X(x)}+\frac{2\left(2 x_{0}+b\right)}{Q} \tan ^{-1} \frac{Q}{2 x+b}\right]\right\},
\end{aligned}
$$

where $x=r_{s}^{1 / 2}, X(x)=x^{2}+b x+c, Q=\left(4 c-b^{2}\right)^{1 / 2}$, and $A, b, c$, and $x_{0}$ are parameters. For an explanation of this form, see Ref. [113]. 
The best available analytic representation of the Ceperley-Alder data was devised by Perdew and Wang [114] (PW92)

$$
\epsilon_{\mathrm{c}}^{\mathrm{PW} 92, i}\left(r_{s}\right)=-2 A\left(1+\alpha_{1} r_{s}\right) \ln \left[1+\frac{1}{2 A\left(\beta_{1} r_{s}^{1 / 2}+\beta_{2} r_{s}+\beta_{3} r_{s}^{3 / 2}+\beta_{4} r_{s}^{2}\right)}\right],
$$

where $A, p, \alpha_{1}, \beta_{1}, \beta_{2}, \beta_{3}$, and $\beta_{4}$ are parameters assuming different values for each $\epsilon_{c}^{i}\left(r_{s}\right)$. Unlike the VWN form, form (74) properly allows for a nonzero coefficient $C$ in Eq. (69) and avoids other deficiencies of the PZ81 and VWN parametrizations.

Even with accurate representations of $\epsilon_{\mathrm{c}}^{P}\left(r_{s}\right)$ and $\epsilon_{\mathrm{c}}^{F}\left(r_{s}\right)$ at our disposal, we need a general formula applicable to spin-polarized systems. Without loss of generality we can assume that, in analogy with Eq. (62),

$$
E_{\mathrm{c}}^{\mathrm{LSDA}}\left[\rho_{\alpha}, \rho_{\beta}\right]=\int \rho \epsilon_{\mathrm{c}}\left(r_{s}, \zeta\right) d \mathbf{r}
$$

where the function $\epsilon_{\mathrm{c}}\left(r_{s}, \zeta\right)$ is to be determined. Unfortunately, unlike for exchange, there is no simple exact formula relating $\epsilon_{\mathrm{c}}\left(r_{s}, \zeta\right)$ to $\epsilon_{\mathrm{c}}^{P}\left(r_{s}\right), \epsilon_{\mathrm{c}}^{F}\left(r_{s}\right)$, and $\zeta$. Von Barth and Hedin [56] proposed using the same interpolation formula for $\epsilon_{\mathrm{c}}\left(r_{s}, \zeta\right)$ as for $\epsilon_{\mathrm{x}}(\rho, \zeta)$, that is,

$$
\epsilon_{\mathrm{c}}^{\mathrm{BH}}\left(r_{s}, \zeta\right)=\epsilon_{\mathrm{c}}^{P}\left(r_{s}\right)+\left[\epsilon_{\mathrm{c}}^{F}\left(r_{s}\right)-\epsilon_{\mathrm{c}}^{P}\left(r_{s}\right)\right] f(\zeta),
$$

where $f(\zeta)$ is given by Eq. (67). In practice, Eq. (76) is not very accurate [45]. Vosko, Wilk, and Nusair [113] examined several alternatives to the Barth-Hedin interpolation formula and recommended the following expression

$$
\epsilon_{\mathrm{c}}^{\mathrm{VWN}}\left(r_{s}, \zeta\right)=\epsilon_{\mathrm{c}}^{P}\left(r_{s}\right)+\alpha_{\mathrm{c}}\left(r_{s}\right)\left[\frac{f(\zeta)}{f^{\prime \prime}(0)}\right]\left(1-\zeta^{4}\right)+\left[\epsilon_{\mathrm{c}}^{F}\left(r_{s}\right)-\epsilon_{\mathrm{c}}^{P}\left(r_{s}\right)\right] f(\zeta) \zeta^{4},
$$

where $\alpha_{\mathrm{c}}\left(r_{s}\right)$ is a new function called spin stiffness. The spin stiffness is formally defined as $\alpha_{\mathrm{c}}\left(r_{s}\right)=\left[\partial^{2} \epsilon_{\mathrm{c}}\left(r_{s}, \zeta\right) / \partial \zeta^{2}\right]_{\zeta=0}$ and fitted to the same analytic form as $\epsilon_{\mathrm{c}}^{P}\left(r_{s}\right)$ and $\epsilon_{\mathrm{c}}^{F}\left(r_{s}\right)$.

The PW92 parametrization of the LDA correlation energy of Eq. (74) combined with VWN interpolation formula (77) is the most accurate representation of the LSDA correlation energy functional currently available. ${ }^{2}$ It is used as part of the PW91 correlation functional implemented in the GAUSSIAN program [32]. ${ }^{3}$ The PZ81 parametrization of $\epsilon_{\mathrm{c}}^{i}\left(r_{s}\right)$ in combination with the Barth-Hedin interpolation formula (76) is used in the GAUSSIAN implementation of the Perdew 1986 correlation functional (P86) and as a standalone LDA correlation functional (keyword PL). The VWN parametrization of $\epsilon_{\mathrm{c}}^{i}\left(r_{s}\right)$ of Eq. (73) is meant to be used with the VWN interpolation formula (77).

\section{Density-gradient expansion}

Although the LSDA is exact for a uniform electron gas and quite accurate for solids, it is less than satisfactory for atoms and molecules. The natural step beyond the LDA is a formal

\footnotetext{
${ }^{2}$ In the GAUSSIAN program [32], the keyword LSDA requests the Barth-Hedin interpolation formula in which $\epsilon_{\mathrm{c}}^{P}\left(r_{s}\right)$ and $\epsilon_{\mathrm{c}}^{F}\left(r_{s}\right)$ have the analytic form of Eq. (73) but employ parameters that were fitted to the correlation energy of a uniform electron gas calculated in the random-phase approximation (RPA).

${ }^{3}$ Currently there is no stand-alone keyword for the PW92 representation of LSDA in the GAUSSIAN program. In GAUSSIAN 03, one can perform LSDA-PW92 calculations by specifying the combination of keywords SPW91 IOp (3/78=0000010000) in the input section.
} 
expansion of $E_{\mathrm{xc}}$ in gradients of the density. This idea was suggested already by Hohenberg and Kohn [15]. In general, the density-gradient expansion (DGE) of the exchange-correlation energy has the form of Eq. (52), but the requirement that $E_{\mathrm{xc}}$ be invariant under rotations about $\mathbf{r}$ makes the coefficients of $\nabla \rho$ and other nonscalar terms vanish, yielding [15]

$$
E_{\mathrm{xc}}[\rho]=\int \rho \epsilon_{\mathrm{xc}}^{\mathrm{LDA}}(\rho) d \mathbf{r}+\int|\nabla \rho|^{2} \epsilon_{\mathrm{xc}}^{(2)}(\rho) d \mathbf{r}+\ldots
$$

where $\epsilon_{\mathrm{xc}}^{(2)}(\rho)$ are coefficients of appropriate dimensionality. The $\nabla^{2} \rho$ term in Eq. (78) has been eliminated via integration by parts.

It is customary to discuss gradient expansions for exchange in terms of dimensionless reduced density gradients

$$
x=\frac{|\nabla \rho|}{\rho^{4 / 3}}, \quad s=\frac{x}{2\left(3 \pi^{2}\right)^{1 / 3}} .
$$

The form of the gradient expansion for $E_{\mathrm{x}}$ is fixed by dimensional analysis:

$$
E_{\mathrm{x}}[\rho]=E_{\mathrm{x}}^{\mathrm{LDA}}[\rho]-\beta_{\mathrm{x}} \int \rho^{4 / 3} x^{2} d \mathbf{r}+\ldots,
$$

where $\beta_{\mathrm{x}}$ is the second-order DGE coefficient. Equivalently, Eq. (80) can be written as

$$
\begin{aligned}
E_{\mathrm{x}}[\rho] & =\int \rho \epsilon_{\mathrm{x}}^{\mathrm{LDA}}(\rho)\left[1+\frac{\beta_{\mathrm{x}}}{C_{\mathrm{x}}} x^{2}+\ldots\right] d \mathbf{r} \\
& =\int \rho \epsilon_{\mathrm{x}}^{\mathrm{LDA}}(\rho)\left[1+\mu_{\mathrm{x}} s^{2}+\ldots\right] d \mathbf{r} .
\end{aligned}
$$

The coefficients of $s^{2}$ and $x^{2}$ are related by

$$
\mu_{\mathrm{x}}=\frac{\left[2\left(3 \pi^{2}\right)^{1 / 3}\right]^{2}}{C_{\mathrm{x}}} \beta_{\mathrm{x}} .
$$

Derivation of gradient expansion coefficients is very involved even for the second-order terms [115-118]. According to Antoniewicz and Kleinman [115], the exact second-order coefficient in Eq. (81) is

$$
\mu_{\mathrm{x}}=\mu_{\mathrm{AK}}=\frac{10}{81}
$$

which is different from Sham's result [116]

$$
\mu_{\mathrm{x}}=\mu_{\mathrm{S}}=\frac{7}{81}, \quad \beta_{\mathrm{S}}=0.00166721 \ldots
$$

Coefficients of the fourth-order terms in the DGE for the exchange energy for slowly-varying densities have been obtained only recently [119, 120].

The gradient expansion for the correlation energy begins as

$$
\begin{aligned}
E_{\mathrm{c}}[\rho] & =E_{\mathrm{c}}^{\mathrm{LDA}}[\rho]+\int C_{\mathrm{c}}\left(r_{s}\right) \rho^{4 / 3} x^{2} d \mathbf{r}+\ldots \\
& =\int \rho\left[\epsilon_{\mathrm{c}}^{\mathrm{LDA}}(\rho)+\beta_{\mathrm{c}}(\rho) t^{2}+\ldots\right] d \mathbf{r},
\end{aligned}
$$


where

$$
t=\frac{1}{4(3 / \pi)^{1 / 6}} \frac{|\nabla \rho|}{\rho^{7 / 6}} .
$$

The value of the coefficient $\beta_{\mathrm{c}}$ for the high-density limit was obtained analytically by Ma and Brueckner [121] (in a.u.)

$$
\lim _{\rho \rightarrow \infty} \beta_{\mathrm{c}}=\beta_{\mathrm{MB}}=16(3 / \pi)^{1 / 3} C_{\mathrm{c}}(0) \approx 1.97563 / 3 \pi^{2}=0.0667244 \ldots
$$

An analytic representation of the function $C_{\mathrm{c}}\left(r_{s}\right)$ has been given by Rasolt and Geldart [122]. Observe that Eqs. (81) and (85) correctly reduce to LDA for uniform densities.

The second-order density-gradient expansion improving upon the LTA of Eq. (68) has been derived by Ernzerhof and Scuseria [123]

$$
E_{\mathrm{x}}^{\tau \mathrm{DGE}}[\tau]=E_{\mathrm{x}}^{\mathrm{LTA}}[\tau]+\beta_{\mathrm{ES}} \int \tau^{4 / 5}\left(\frac{|\nabla \tau|}{\tau^{6 / 5}}\right)^{2} d \mathbf{r}
$$

where is $\beta_{\mathrm{ES}}$ is a nonempirical coefficient. Because $\beta_{\mathrm{ES}}$ turns out to be positive, Eq. (88) can give unphysical positive exchange energies.

The second-order DGE results only in modest improvements over LDA for exchange energies. Herman et al. [124] found empirically that the optimal value of the second-order gradient coefficient $\beta_{\mathrm{x}}$ in the second-order DGE approximation is about 2.5 times greater than the exact value. For correlation, the second-order DGE overestimates the correction needed to reproduce the exact correlation energy by a factor of 5 and so predicts positive correlation energies [121]. This implies that density variations in atoms and molecules are too rapid to be approximated by Eqs. (81) and (85). Because of such problems, truncated density-gradient expansions are not used as practical density functionals. Instead, they are regarded as the exact forms to which approximate exchange-correlation functionals should reduce in the limit of slowly-varying densities.

\section{Constraint satisfaction}

Improvement of the second-order density gradient expansion became the focus of many research efforts in the 1970s and 1980s. Some developers of density functionals [125-132] saw the root of problems in the wrong analytic behavior of the exchange-correlation potential of the DGE functional and concentrated on designing semiempirical gradient corrections with desired analytic properties. Others [133-135] explained failures of the second-order DGE (divergence of the exchange-correlation potential, overcorrection of the correlation energy, etc.) by the fact that the exchange-correlation hole corresponding to the second-order DGE exhibits undamped oscillation at large $u$ values and thereby severely violates the important normalization constraints and sign properties of the correct hole. Accordingly, functionals based on explicitly normalized and properly signed exchange-correlation holes have been proposed. It is not hard to see that the two approaches are essentially different routes to the same goal: satisfaction of important exact constraints. As we shall see in this section, the process of identifying important analytic properties of the exact exchange-correlation functional and imposing them on approximate constructions is the most reliable strategy of density functional design. 


\subsection{Corrections on the asymptotic behavior}

The second-order density gradient expansion for exchange performs well only in the limit of small reduced density gradients $x$. The assumption that $x$ is small may be justified for an infinite electron gas but not for finite systems, where $x$ diverges in far-out regions. This can be demonstrated by assuming a spherically symmetric exponential density $\rho(r)=e^{-a r}$,

$$
\lim _{r \rightarrow \infty} x=\lim _{r \rightarrow \infty} \frac{|\partial \rho / \partial r|}{\rho^{4 / 3}}=\lim _{r \rightarrow \infty} a e^{a r / 3}=\infty .
$$

The second-order gradient contribution to the energy density, proportional to $\rho^{4 / 3} x^{2}$ [see Eq. (80)], remains finite but the leading term of the exchange potential $v_{\mathrm{x}}(\mathbf{r})$ [see Eq. (25)] scales like $\rho^{1 / 3} x^{2}$, and so is unbounded at large $r$. Various modifications of the second-order DGE have been suggested to eliminate the asymptotic divergence of $v_{\mathrm{x}}(\mathbf{r})$. Most of these functionals fall into the category of generalized gradient approximations (GGA) [136]

$$
E_{\mathrm{x}}^{\mathrm{GGA}}[\rho]=\int \rho \epsilon_{\mathrm{x}}^{\mathrm{LDA}}(\rho) F_{\mathrm{x}}(x) d \mathbf{r}=\int \rho \epsilon_{\mathrm{x}}^{\mathrm{GGA}}(\rho, x) d \mathbf{r},
$$

in which $F_{\mathrm{x}}(x)$ [or $F_{\mathrm{x}}(s)$ ] approximates the exact enhancement factor of Eq. (81).

One of the first successful functionals of this type was proposed in 1986 by Becke [125]. Its enhancement factor is modeled after that of Eq. (81) and has a damped second-order gradient term

$$
F_{\mathrm{x}}^{\mathrm{B} 86}(s)=1+\frac{\beta c_{2}\left(c_{1} s\right)^{2}}{1+\gamma\left(c_{1} s\right)^{2}},
$$

where $c_{1}=2\left(6 \pi^{2}\right)^{1 / 3}$ and $c_{2}=\left(2^{1 / 3} C_{\mathrm{x}}\right)^{-1}$, while $\beta=0.0036$ and $\gamma=0.004$ are empirical parameters. ${ }^{4}$ Shortly thereafter, Becke observed [126] that the large-gradient $(s \rightarrow \infty)$ limit of Eq. (91) is $\sim s^{0}$, whereas the correct limit should be $\sim s^{2 / 5}$. To ensure the correct behavior both in the low- and high-gradient limits, he proposed the following modification

$$
F_{\mathrm{x}}^{m \mathrm{~B} 86}(s)=1+\frac{\beta c_{2}\left(c_{1} s\right)^{2}}{\left[1+\gamma\left(c_{1} s\right)^{2}\right]^{4 / 5}},
$$

where $\beta=0.00375$ and $\gamma=0.007$ are again optimized parameters.

With an empirical value of $\beta$, neither B86 nor $m$ B86 recovers the correct second-order gradient expansion of Eq. (81). DePristo and Kress [127] explicitly imposed this constraint by using the formula

$$
F_{\mathrm{x}}^{\mathrm{DK} 87}(s)=1+\mu_{\mathrm{S}} s^{2} \frac{1+\gamma_{1}\left(c_{1} s\right)^{m}}{1+\gamma_{2}\left(c_{1} s\right)^{2}},
$$

where $\mu_{\mathrm{S}}=7 / 81$ is Sham's exact second-order coefficient, but $\gamma_{1}, \gamma_{2}$, and $m \leq 1$ are still adjustable parameters. Note that the large-gradient limit of Eq. (93) is $\sim s^{m}$, which is correct only if $m=2 / 5$.

\footnotetext{
${ }^{4}$ The factors $c_{1}=2\left(6 \pi^{2}\right)^{1 / 3}$ and $c_{2}=\left(2^{1 / 3} C_{\mathrm{x}}\right)^{-1}$ in Eqs. (91)-(93), (96), (99), (101), (110), and (180) arise in the transition from the $E_{\mathrm{x}}\left[\rho_{\alpha}, \rho_{\beta}\right]$ form of the original definitions, where $F_{\mathrm{x}}$ is written in terms of $x_{\sigma}=\left|\nabla \rho_{\sigma}\right| / \rho_{\sigma}^{4 / 3}$, to the standard form $E_{\mathrm{x}}[\rho]$, where $F_{\mathrm{x}}$ is written in terms of $s$ only. The transformation is based on the spin-scaling relation of Eq. (50). Note that $c_{1} s=2^{1 / 3} x$.
} 
It is also known that in a finite many-electron system, the true exchange potential $v_{\mathrm{x}}(\mathbf{r})$ and exchange energy density $e_{\mathrm{x}}(\mathbf{r})$ have the following asymptotic behavior $[45,137]$ :

$$
\left.v_{\mathrm{x}}(\mathbf{r})\right|_{r \rightarrow \infty}=-\frac{1}{r}+C,
$$

where $C$ is a constant that vanishes everywhere except in nodal surfaces of the highest occupied orbital [138], and

$$
\left.e_{\mathrm{x}}(\mathbf{r})\right|_{r \rightarrow \infty}=-\frac{\rho(r)}{2 r} .
$$

Although the B86, mB86, and DK87 exchange potentials no longer diverge and, like energy densities, go to zero in the $r \rightarrow \infty$ limit, their asymptotic decay is not of the form prescribed by Eqs. (94) and (95). Becke [128] argued that the asymptotic limit of $e_{\mathrm{x}}(\mathbf{r})$ is an important constraint and proposed a functional which satisfies Eq. (95) for exponentially decaying densities

$$
F_{\mathrm{x}}^{\mathrm{B} 88}(s)=1+\frac{\beta c_{2}\left(c_{1} s\right)^{2}}{1+6 \beta\left(c_{1} s\right) \sinh ^{-1}\left(c_{1} s\right)},
$$

where $\beta=0.0042$ is an empirical parameter determined by a least squares fit to exact Hartree-Fock exchange energies of six noble gas atoms (He through $\mathrm{Rn}$ ). It is the function $\sinh ^{-1} y=\ln \left(y+\sqrt{1+y^{2}}\right)$ that does the trick of ensuring the correct behavior of $e_{\mathrm{x}}^{\mathrm{B} 88}(\mathbf{r})$ at large $r$. To see this, use the expansion [139]

$$
\sinh ^{-1} y=\ln (2 y)+\frac{1}{2 \cdot 2 y^{2}}-\frac{1 \cdot 3}{2 \cdot 4 \cdot 4 y^{4}}+\ldots, \quad|y|>1
$$

and the large- $r$ form of $c_{1} s=2^{1 / 3} x$, where $x$ is given by Eq. (89). The B88 functional proved very successful and remains one of the most popular approximations for the exchange energy.

In the early 1990s, Lacks and Gordon (LG) [140] observed that the LDA, B86, mB86, DK87, and B88 exchange functionals all give a poor description of van der Waals interactions between rare gas atoms, especially at large internuclear distances. Even at the energy minima, exchange contributions to the binding energy are in errors exceeding $100 \%$. In an effort to refine the description of low-density, high-gradient regions responsible for long-range interactions, they considered the form

$$
F_{\mathrm{x}}^{\mathrm{LG}}(s)=\frac{\left[1+\left(a_{d}+\mu_{\mathrm{AK}}\right) s^{2} / b+a_{4} s^{4}+a_{6} s^{6}+a_{8} s^{8}+a_{10} s^{10}+a_{12} s^{12}\right]^{b}}{1+a_{d} s^{2}},
$$

where $a_{4}, a_{6}, a_{8}, a_{10}, a_{12}$, and $b$ are empirical parameters obtained by least squares fitting to the total exchange energies of the $\mathrm{He}, \mathrm{Ne}$, and $\mathrm{C}^{+}$atoms and the exchange contributions to the binding energies of $\mathrm{He}_{2}$ and $\mathrm{Ne}_{2}$. The value of $a_{d}$ was fixed at $10^{-8}$. In more extensive tests, Adamo and Barone [141] found that the combination of the LG exchange and LYP correlation is not a substantial improvement over BLYP.

In 1997, Filatov and Thiel [142] observed that the correct asymptotic limit of the exchange energy per density is also obtained with functionals of the general form

$$
F_{\mathrm{x}}^{\mathrm{FT} 97}(s)=1+\frac{\beta c_{2}\left(c_{1} s\right)^{2}}{\left[1+(6 \beta / m)^{m}\left(c_{1} s\right)^{m} \sinh ^{-1}\left(c_{1} s\right)^{m}\right]^{1 / m}} .
$$


Using $m=2$ and allowing the adjustable parameter $\beta$ to assume different values for spin-up and spin-down densities, they managed to reduce the mean absolute error in the exchange energy by about one third relative to B88.

Van Leeuwen and Baerends [129] achieved the correct asymptotic behavior of $v_{\mathrm{xc}}(\mathbf{r})$ by modeling the Kohn-Sham exchange-correlation potential directly rather than by approximating the energy density. Their first model $v_{\mathrm{xc}}(\mathbf{r})$ had an analytic form inspired by the B88 functional [129]. More recent shape-corrected potentials of Baerends and coworkers [143-147] involve explicit dependence on Kohn-Sham orbitals and satisfy even more exact constraints, such as invariance with respect to shifting the external potential by a constant and correct short-range behavior. The advantage of modeling $v_{\mathrm{xc}}(\mathbf{r})=v_{\mathrm{x}}(\mathbf{r})+v_{\mathrm{c}}(\mathbf{r})$ is that potentials, unlike energy densities, are uniquely determined by $\rho(\mathbf{r})$. Given an exchange potential $v_{\mathbf{x}}(\mathbf{r})$, the exchange energy can be evaluated by the Levy-Perdew formula [78]. More recently, Tozer and Handy [131, 132] proposed an explicit asymptotic correction for conventional exchangecorrelation potentials and obtained encouraging results for sensitive properties. The correct asymptotic behavior of the exchange energy density and potential appears to be much less important for obtaining accurate atomic exchange energies.

What about satisfying constraints (94) and (95) simultaneously? Engel and coworkers [148] proved that no GGA of the form of Eq. (90) can reproduce the asymptotic limits of Eqs. (94) and (95) at the same time. Jemmer and Knowles [130] and Filatov and Thiel [149] attempted to satisfy Eqs. (94) and (95) simultaneously by going beyond the conventional gradient approximation and introducing the dependence on the Laplacian of the density:

$$
E_{\mathrm{x}}[\rho]=\int \rho \epsilon_{\mathrm{x}}^{\mathrm{LDA}}(\rho) F_{\mathrm{x}}\left(\rho,|\nabla \rho|, \nabla^{2} \rho\right) d \mathbf{r} .
$$

Such functionals suffer, however, from numerical instabilities with respect to small density changes, which makes it practically impossible to obtain variational solutions of the KohnSham equations [130]. Neumann and Handy [150] investigated the possibility of including terms of up to fourth order in $\nabla$ and also arrived at decidedly disappointing conclusions.

Gill [151] disputed the claim that the asymptotic divergence of the exchange potential is of practical significance and argued in favor of a minimalistic empirical functional with a readjusted fractional power of the gradient correction

$$
F_{\mathrm{x}}^{\mathrm{G} 96}(s)=1+\beta c_{2}\left(c_{1} s\right)^{3 / 2},
$$

where $\beta=1 / 137$ is a parameter whose value was chosen to reproduce the Hartree-Fock exchange energy of the Ar atom (one also cannot help suspecting a playful reference to the fine structure constant). The exchange potential corresponding to Eq. (101) again diverges at large $x$. Contrary to what one might expect, G96 shows an accuracy on par with B88 in test calculations of thermochemical molecular properties, a better performance than of some much more sophisticated functionals.

Generalized gradient approximations based on the local- $\tau$ approximation of Eq. (68) have been constructed by Ernzerhof et al. [152]. These workers focused on improving the original LTA of Eq. (68) and second-order $\tau$-DGE of Eq. (88) by imposing several important constraints on the enhancement factor: (a) the correct homogeneous density limit; (b) negativity of $E_{\mathrm{x}}$; (c) correct asymptotic behavior of the exchange energy density [Eq. (95)]. The resulting $\tau$-GGA shows a performance comparable to that of conventional GGA functionals. 
Intuitively designed damped gradient corrections have been also used to improve the LDA for correlation. The first attempt of this kind was made by Ma and Brueckner [121] in their paper on the exact second-order expansion of $E_{\mathrm{c}}[\rho]$, where they also propose the functional

$$
E_{\mathrm{c}}^{\mathrm{MB} 68}[\rho]=\int \rho \epsilon_{\mathrm{c}}^{\mathrm{LDA}}(\rho)\left[1-\beta_{\mathrm{MB}} \frac{t^{2}}{\nu \rho \epsilon_{\mathrm{c}}^{\mathrm{LDA}}(\rho)}\right]^{-\nu} d \mathbf{r} .
$$

The constant $\nu=0.32$ was fitted to the empirical correlation energies of several atoms.

\subsection{Normalization of the exchange-correlation hole}

Similar to the partitioning of $E_{\mathrm{xc}}$ into exchange and correlation contributions, the exchangecorrelation hole at a coupling strength $\lambda$ can be partitioned as

$$
h_{\mathrm{xc}}^{\lambda}\left(\mathbf{r}_{1}, \mathbf{r}_{2}\right)=h_{\mathrm{x}}\left(\mathbf{r}_{1}, \mathbf{r}_{2}\right)+h_{\mathrm{c}}^{\lambda}\left(\mathbf{r}_{1}, \mathbf{r}_{2}\right) \text {, }
$$

where the exchange hole is defined as the noninteracting limit

$$
h_{\mathrm{x}}\left(\mathbf{r}_{1}, \mathbf{r}_{2}\right) \equiv h_{\mathrm{xc}}^{\lambda=0}\left(\mathbf{r}_{1}, \mathbf{r}_{2}\right) .
$$

The basic properties of the exact exchange and correlation holes are:

$$
\begin{gathered}
h_{\mathrm{x}}\left(\mathbf{r}_{1}, \mathbf{r}_{2}\right) \leq 0, \quad \int h_{\mathrm{x}}\left(\mathbf{r}_{1}, \mathbf{r}_{2}\right) d \mathbf{r}_{2}=-1, \\
\int h_{\mathrm{c}}^{\lambda}\left(\mathbf{r}_{1}, \mathbf{r}_{2}\right) d \mathbf{r}_{2}=0 .
\end{gathered}
$$

Langreth and Perdew [133] and Perdew [135] explained the failure of the second-order DGE by a gross violation of Eqs. (105) and (106) by the DGE exchange and correlation holes. Such violations have been traced to spurious large- $u$ behavior of the sphericallyaveraged $h_{\mathrm{xc}}(\mathbf{r}, u)$. Perdew showed [135] that by cutting off large- $u$ parts of $h_{\mathrm{xc}}(\mathbf{r}, u)$ and positive parts of $h_{\mathrm{x}}(\mathbf{r}, u)$ one can obtain successful density functional approximations. The essence of the cutoff procedure for exchange in real space is as follows [153-155].

Start with the analytic second-order density gradient expansion for the coupling constantand angle-averaged exchange hole, $h_{\mathrm{x}}^{\mathrm{DGE}}(\mathbf{r}, u)$. Convert the diverging DGE hole into the normalized GGA hole by applying a sharp cutoff:

$$
h_{\mathrm{x}}^{\mathrm{GGA}}(\mathbf{r}, u)=h_{\mathrm{x}}^{\mathrm{DGE}}(\mathbf{r}, u) \theta\left(h_{\mathrm{x}}^{\mathrm{DGE}}\right) \theta\left(u_{0}(\mathbf{r})-u\right),
$$

where

$$
\theta(y)= \begin{cases}1, & \text { if } y \geq 0 \\ 0, & \text { if } y<0\end{cases}
$$

The first step function in Eq. (107) enforces the negativity constraint. The second function involves a cutoff radius $u_{0}(\mathbf{r})$ chosen to satisfy the hole normalization constraint of Eq. (105). The hole of Eq. (108) is substituted into Eq. (33) to give the numerical enhancement factor $F_{\mathrm{x}}(x)$, which is then fitted to an analytic form. ${ }^{5}$ The real-space cutoff procedure determines

\footnotetext{
${ }^{5}$ Strictly speaking, the cutoff procedure uses the angle- and system-averaged GGA exchange hole [156], i.e., the hole around an electron averaged over the position of that electron. We omit here technical details for the sake of clarity.
} 
only the general features of the functional but not its analytic representation. The latter may be chosen in different ways depending on which exact constraints one decides to impose. The first practical exchange functional of this type was derived in 1986 by Perdew and Wang [136]

$$
F_{\mathrm{x}}^{\mathrm{PW} 86}(s)=\left(1+\frac{\mu_{\mathrm{S}} s^{2}}{m}+b s^{4}+c s^{6}\right)^{m}
$$

in which $m=1 / 15, \mu_{\mathrm{S}}=7 / 81, b=14$, and $c=0.2$ are nonempirical parameters.

In 1991, Perdew and Wang $[76,155,157]$ used the same approach to derive a nonempirical exchange functional whose analytic representation was inspired by the B88 form:

$$
F_{\mathrm{x}}^{\mathrm{PW} 91}(s)=1+\frac{\beta c_{2}\left(c_{1} s\right)^{2}-\left[\beta c_{2}\left(c_{1} s\right)^{2}-\mu_{\mathrm{AK}}\right] e^{-100 s^{2}}-0.004 s^{4}}{1+6 \beta\left(c_{1} s\right) \sinh ^{-1}\left(c_{1} s\right)+0.004 s^{4}},
$$

where $c_{1}=2\left(6 \pi^{2}\right)^{1 / 3}, c_{2}=\left(2^{1 / 3} C_{\mathrm{x}}\right)^{-1}, \beta=0.0042$ (the B88 coefficient), and $\mu_{\mathrm{AK}}=10 / 81$. The particular form of Eq. (110) was chosen to satisfy a large number of exact constraints. Although PW91 is modeled after the B88 functional, it does not yield the correct asymptotic behavior of the exchange energy density, a property abandoned in favor of more desirable constraints. The function $\sinh ^{-1}\left(c_{1} s\right)$ in Eq. (110) is only a B88 relic.

The exact density-gradient expansion for the correlation hole is not known except in the high-density limit, so away from this limit approximate gradient-corrected models have to be used instead. Perdew and coworkers [153,158] constructed a real-space model for the spherically-averaged GGA correlation hole starting from an accurate analytic representation $A_{\mathrm{c}}\left(r_{s}, \zeta, u\right)$ of the LSDA correlation hole, adding a gradient correction $B_{\mathrm{c}}\left(r_{s}, \zeta, u\right)$, and truncating the sum to satisfy the normalization constraint of Eq. (106)

$$
\bar{h}_{\mathrm{c}}^{\mathrm{GGA}}\left(r_{s}, \zeta, t, u\right)=\phi^{3}\left(\phi k_{s}\right)^{2}\left[A_{\mathrm{c}}\left(r_{s}, \zeta, u\right)+t^{2} B_{\mathrm{c}}\left(r_{s}, \zeta, u\right)\right] \theta\left(u_{0}-u\right),
$$

where $k_{s}=\left(4 k_{F} / \pi\right)^{1 / 2}, t$ is the reduced gradient of Eq. (86) generalized to spin-polarized systems

$$
t=\frac{|\nabla \rho|}{2 \phi k_{s} \rho}
$$

$u_{0}$ is the cutoff radius, and

$$
\phi(\zeta)=\frac{(1+\zeta)^{2 / 3}+(1-\zeta)^{2 / 3}}{2}
$$

The hole of Eq. (111) is substituted into Eq. (33) and the integral is evaluated numerically. The result is written as

$$
E_{\mathrm{c}}^{\mathrm{GGA}}\left[\rho_{\alpha}, \rho_{\beta}\right]=\int \rho \epsilon_{\mathrm{c}}^{\mathrm{LSDA}}\left(r_{s}, \zeta\right) d \mathbf{r}+\int \rho H\left(r_{s}, \zeta, t\right) d \mathbf{r},
$$

where $H\left(r_{s}, \zeta, t\right)$ is a numerically defined gradient correction. The last step in this derivation is to find a suitable analytic representation for $H\left(r_{s}, \zeta, t\right)$. Two different solutions to this problem have been proposed, leading to the PW91 [76, 155, 157] and PBE [159] correlation functionals. The PW91 gradient correction has the form

$$
H^{\mathrm{PW} 91}\left(r_{s}, \zeta, t\right)=H_{0}^{\mathrm{PW} 91}\left(r_{s}, \zeta, t\right)+H_{1}\left(r_{s}, \zeta, t\right) .
$$


The $H_{1}$ term in Eq. (115) is negligible, unless $s \ll 1$ [155]. In PBE,

$$
H^{\mathrm{PBE}}\left(r_{s}, \zeta, t\right)=H_{0}^{\mathrm{PBE}}\left(r_{s}, \zeta, t\right) .
$$

The analytic representations of $H_{0}^{\mathrm{PW} 91}$ and $H_{0}^{\mathrm{PBE}}$ have the same form given by

$$
H_{0}\left(r_{s}, \zeta, t\right)=\gamma \phi^{3}(\zeta) \ln \left[1+\frac{\beta_{\mathrm{MB}}}{\gamma} t^{2}\left(\frac{1+A t^{2}}{1+A t^{2}+A^{2} t^{4}}\right)\right]
$$

where

$$
A=\frac{\beta_{\mathrm{MB}}}{\gamma} \frac{1}{e^{-\epsilon_{\mathrm{c}}^{\mathrm{LSDA}}\left(r_{s}, \zeta\right) / \gamma \phi^{3}}-1} .
$$

The only difference between $H_{0}^{\mathrm{PW} 91}$ and $H_{0}^{\mathrm{PBE}}$ is in the values of $\gamma$ used in Eqs. (117) and (118). In PW91,

$$
\gamma_{\mathrm{PW} 91}=\frac{\beta_{\mathrm{MB}}^{2}}{2 \alpha} \approx 0.024734
$$

where $\alpha=0.09$ is a constant chosen to approximate the $t^{4}$ dependence of the numerical $\epsilon_{\mathrm{c}}^{\mathrm{GGA}}$. In PBE,

$$
\gamma_{\mathrm{PBE}}=A_{\mathrm{GB}} \approx 0.031091,
$$

which is the exact second-order gradient coefficient of the LDA correlation energy expansion [see Eq. (70)].

The analytic form of the gradient correction $H\left(r_{s}, \zeta, t\right)$ was motivated by the following three conditions [159]: (1) $H$ must reduce to the second-order term in the DGE expansion for correlation in the slowly-varying limit, $H \rightarrow \beta_{\mathrm{MB}} \phi^{3} t^{2}$; (2) if the density changes infinitely fast $(t \rightarrow \infty)$, the correlation energy must vanish, that is, $H \rightarrow-\epsilon_{\mathrm{c}}^{\mathrm{LSDA}} ;(3)$ under uniform scaling to the high-density limit, $H$ must cancel the logarithmic singularity of $\epsilon_{\mathrm{c}}^{\mathrm{LDA}}$ to satisfy the uniform scaling constraint of Eq. (49).

The $H_{0}^{\mathrm{PBE}}$ recovers the first constraint by itself, but $H_{0}^{\mathrm{PW} 91}$ does not. In order to recover the correct second-order gradient expansion for the correlation energy in the $s \rightarrow 0$ limit, another term is used:

$$
H_{1}\left(r_{s}, \zeta, t\right)=\nu\left[C_{\mathrm{c}}\left(r_{s}\right)-C_{\mathrm{c}}(0)+\frac{3}{7} \beta_{\mathrm{S}}\right] \phi^{3}(\zeta) t^{2} e^{-100 \phi^{4}(\zeta)\left(k_{s} t / k_{F}\right)^{2}}
$$

where $\nu=16(3 / \pi)^{1 / 3}$ is the conversion factor from the units of $\rho t^{2}$ to $\rho^{4 / 3} x^{2}$.

The numerical GGA based on the model hole of Eq. (111) and the PBE analytic representation of this functional both satisfy Levy's uniform scaling constraint for correlation, but the form $E_{\mathrm{c}}^{\mathrm{PW} 91}\left[\rho_{\alpha}, \rho_{\beta}\right]$ does not (the culprit here is the small $H_{1}$ term). In molecular calculations, however, these subtle differences never show up, so the PBE and PW91 correlation functionals are equivalent for most practical purposes.

\subsection{Systematic constraint satisfaction}

Simply imposing correct asymptotic limits on the second-order density-gradient expansion (Section 7.1) proved to be a very effective strategy for designing exchange functionals. It is reasonable to assume that the more exact constraints an approximate density functional 
satisfies, the more accurate and universal it will be. This idea is behind many existing density functional approximations.

Langreth and Mehl [134] used the sharp cut-off procedure in momentum space to eliminate spurious contributions to $E_{\mathrm{c}}$ and an empirical exponential function to damp the gradient contribution to the energy. The Langreth-Mehl (LM) functional $[134,160,161]$ has now mostly a historical significance. A few years later, Perdew [162] improved the LM functional by imposing two additional requirements that it recover the correct second-order DGE in the slowly-varying density limit and reduce in the uniform density limit to LDA, not to the random-phase approximation, as the LM functional does. Perdew's 1986 correlation functional is

$$
E_{\mathrm{c}}^{\mathrm{P} 86}\left[\rho_{\alpha}, \rho_{\beta}\right]=E_{\mathrm{c}}^{\mathrm{LSDA}}\left[\rho_{\alpha}, \rho_{\beta}\right]+\int \frac{e^{-\Phi(\rho, \nabla \rho)}}{d(\zeta)} C_{\mathrm{c}}\left(r_{s}\right) \frac{|\nabla \rho|^{2}}{\rho^{4 / 3}} d \mathbf{r},
$$

where the function

$$
d(\zeta)=2^{1 / 3}\left[\left(\frac{1+\zeta}{2}\right)^{5 / 3}+\left(\frac{1-\zeta}{2}\right)^{5 / 3}\right]^{1 / 2}
$$

interpolates between the spin-compensated and spin-polarized forms [160], while

$$
\Phi(\rho, \nabla \rho)=(9 \pi)^{1 / 6} \tilde{f} \frac{C_{\mathrm{c}}(0)}{C_{\mathrm{c}}\left(r_{s}\right)} \frac{|\nabla \rho|}{\rho^{7 / 6}}
$$

with the parameter $\tilde{f}=0.11$ chosen to fit the exact correlation energy of the neon atom. $C_{\mathrm{c}}\left(r_{s}\right)$ is the gradient coefficient of Eq. (85) in the parametrization by Rasolt and Geldart [122]. In the original paper, $E_{\mathrm{c}}^{\mathrm{LSDA}}\left[\rho_{\alpha}, \rho_{\beta}\right]$ was taken in the PZ81 parametrization of the Ceperley-Alder data, and this is how P86 is implemented in the GAUSSIAN program. The P86 correlation functional correctly reduces to LDA for uniform densities, but does not scale to a constant under the uniform scaling transformation of the density.

Wilson and Levy [163] were the first to explicitly impose the high-density scaling constraint of Eq. (49) on an approximate correlation functional

$$
E_{\mathrm{c}}^{\mathrm{WL}}\left[\rho_{\alpha}, \rho_{\beta}\right]=\int \rho \frac{(a+b x)\left(1-\zeta^{2}\right)^{1 / 2}}{c+d\left(x_{\alpha}+x_{\beta}\right)+r_{s}} d \mathbf{r}
$$

where $x=|\nabla \rho| / \rho^{4 / 3}, x_{\sigma}=\left|\nabla \rho_{\sigma}\right| / \rho_{\sigma}^{4 / 3}$, and $a, b, c$, and $d$ are empirical parameters. Some earlier correlation functionals, such as B88c and LYP (see below), also respect Eq. (49) for all densities, although they predate the formal proof of it. More recent nonempirical functionals described in the rest of this section are purposely constructed to satisfy Eq. (49) among many other exact constraints. Satisfaction of density scaling constraints for the exchange and correlation energies may not be important for "usual" systems, but it becomes crucial for a proper description of high- $Z$ atomic ions [164].

In 1996, Perdew, Burke and Ernzerhof (PBE) [159] constructed a simple exchange functional by imposing several energetically significant exact constraints. The enhancement factor of the PBE exchange functional is similar to that of B86 [Eq. (91)],

$$
F_{\mathrm{x}}^{\mathrm{PBE}}(s)=1+\frac{\mu s^{2}}{1+\mu s^{2} / \kappa} \text {. }
$$


Here $\kappa=0.804$ is a nonempirical parameter chosen to satisfy the Lieb-Oxford bound in its local form: $F_{\mathrm{x}}(s) \leq 1.804$ for any $s$. The value of $\mu=\beta_{\mathrm{MB}}\left(\pi^{2} / 3\right) \approx 0.21951$ is determined from the condition that the second-order gradient term for exchange cancel that for correlation (i.e., $\rho \beta_{\mathrm{MB}} t^{2}=\mu C_{\mathrm{x}} \rho^{4 / 3} s^{2}$ ). This choice, rather than $\mu=\mu_{\mathrm{AK}}=\frac{10}{81}$, assures that in the $s \rightarrow 0$ limit the PBE exchange-correlation reduces to LDA for nearly uniform densities faster than PW91 does. Although Eq. (126) is not based on any particular hole model, it is numerically very similar to the PW91 exchange functional of Eq. (110) and, in fact, produces nearly the same results. An empirical adjustment of the parameter $\kappa$ from 0.804 to 1.245 in the PBE exchange functional has been proposed [165], but Perdew and coworkers [166] defended the original nonempirical value. Hammer et al. [167] attempted to reconcile both points of view by suggesting a minor revision of the analytic form of Eq. (126).

The PW91 and PBE exchange-correlation approximations exhaust the number of exact constraints that can be practically imposed on GGAs, that is, functionals whose ingredients are $\rho_{\sigma}(\mathbf{r})$ and $\nabla \rho_{\sigma}(\mathbf{r})$. Bringing into play the kinetic energy density $\tau_{\sigma}(\mathbf{r})$ of Eq. (40) opens new opportunities (the meta-GGA level). One particular use of $\tau_{\sigma}(\mathbf{r})$ is based on the following property:

$$
\tau_{\sigma}^{W}(\mathbf{r}) \leq \tau_{\sigma}(\mathbf{r})
$$

where

$$
\tau_{\sigma}^{W}=\frac{1}{8} \frac{\left|\nabla \rho_{\sigma}\right|^{2}}{\rho_{\sigma}}
$$

is the von Weizsäcker kinetic energy density. ${ }^{6}$ In Eq. (127), the strict equality holds only if $\rho_{\sigma}(\mathbf{r})$ is represented by a single real Kohn-Sham orbital. Therefore, the quantity

$$
\eta_{\sigma}^{\mathrm{SCC}}=1-\frac{\tau_{\sigma}^{W}}{\tau_{\sigma}}
$$

vanishes for any one-electron system and is strictly positive in systems with more than one $\sigma$-spin electron $[168,169]$. In practical terms, this means that $\eta_{\sigma}^{\mathrm{SCC}}$ can be used as a multiplicative self-correlation correction (SCC) ensuring that $E_{\mathrm{c}}$ vanishes for any oneelectron density [Eq. (45)]. Satisfaction of this constraint is not possible at the GGA level. The functionals discussed in the rest of this section all make use of this trick.

Becke's 1995 correlation functional (B95) [168] was constructed to satisfy the following set of conditions: (a) the correct uniform density limit; (b) separation of the correlation energy into parallel-spin and opposite-spin components; (c) zero correlation energy for one-electron systems; (d) good fit to the atomic correlation energies. These requirements are met by the following analytic form

$$
E_{\mathrm{c}}^{\mathrm{B} 95}=E_{\mathrm{c}}^{\alpha \alpha}+E_{\mathrm{c}}^{\beta \beta}+E_{\mathrm{c}}^{\alpha \beta},
$$

where

$$
E_{\mathrm{c}}^{\sigma \sigma}=\int \frac{\tau_{\sigma}-\tau_{\sigma}^{W}}{\tau_{\sigma}^{\text {unif }}} \frac{e_{\mathrm{c} \sigma \sigma}^{\mathrm{UEG}}\left(\rho_{\sigma}\right)}{\left(1+c_{\sigma \sigma} x_{\sigma}^{2}\right)^{2}} d \mathbf{r},
$$

${ }^{6}$ Proof. Let us introduce the symbolic vectors $\boldsymbol{f} \equiv\left(\phi_{1}, \phi_{2}, \ldots, \phi_{N}\right)$ and $\boldsymbol{g} \equiv\left(\nabla \phi_{1}, \nabla \phi_{2}, \ldots, \nabla \phi_{N}\right)$, where the spin-index $\sigma$ has been suppressed for brevity. By definition

$$
\rho=|\boldsymbol{f}|^{2}, \quad \tau=\frac{1}{2}|\boldsymbol{g}|^{2}, \quad \tau_{W}=\frac{1}{8} \frac{|\nabla \rho|^{2}}{\rho}=\frac{1}{8} \frac{\left|\boldsymbol{g}^{*} \cdot \boldsymbol{f}+\boldsymbol{f}^{*} \cdot \boldsymbol{g}\right|^{2}}{|\boldsymbol{f}|^{2}} \leq \frac{1}{2} \frac{\left|\boldsymbol{f}^{*} \cdot \boldsymbol{g}\right|^{2}}{|\boldsymbol{f}|^{2}},
$$

where the strict equality holds only if all $\phi_{i}$ are real. The result $\tau_{W} \leq \tau$ follows from the Schwarz inequality $\left|\boldsymbol{f}^{*} \cdot \boldsymbol{g}\right|^{2} \leq|\boldsymbol{f}|^{2}|\boldsymbol{g}|^{2}$. Thus, if $N=1$ and $\phi_{1}$ is real, then $\left|\boldsymbol{f}^{*} \cdot \boldsymbol{g}\right|^{2}=|\boldsymbol{f}|^{2}|\boldsymbol{g}|^{2}$, and so $\tau_{W}=\tau$. 


$$
E_{\mathrm{c}}^{\alpha \beta}=\int \frac{e_{\mathrm{c} \alpha \beta}^{\mathrm{UEG}}\left(\rho_{\alpha}, \rho_{\beta}\right)}{1+c_{\alpha \beta}\left(x_{\alpha}^{2}+x_{\beta}^{2}\right)} d \mathbf{r},
$$

in which $\tau_{\sigma}^{\text {unif }}=\frac{3}{10}\left(6 \pi^{2}\right)^{2 / 3} \rho_{\sigma}^{5 / 3}$, and $c_{\sigma \sigma}=0.038$ and $c_{\alpha \beta}=0.0031$ are fitted parameters. The same-spin correlation energy density of the uniform electron gas (UEG) is simply

$$
e_{\mathrm{c} \sigma \sigma}^{\mathrm{UEG}}\left(\rho_{\sigma}\right)=e_{\mathrm{c}}^{\mathrm{LSDA}}\left(\rho_{\sigma}, 0\right) .
$$

The opposite-spin energy density is freed from self-correlation by the method of Stoll et al. $[170,171]$ :

$$
e_{\mathrm{c} \alpha \beta}^{\mathrm{UEG}}\left(\rho_{\alpha}, \rho_{\beta}\right)=e_{\mathrm{c}}^{\mathrm{LSDA}}\left(\rho_{\alpha}, \rho_{\beta}\right)-e_{\mathrm{c}}^{\mathrm{LSDA}}\left(\rho_{\alpha}, 0\right)-e_{\mathrm{c}}^{\mathrm{LSDA}}\left(\rho_{\beta}, 0\right),
$$

where $e_{\mathrm{c}}^{\mathrm{LSDA}}\left(\rho_{\alpha}, \rho_{\beta}\right)=\rho \epsilon_{\mathrm{c}}^{\mathrm{LSDA}}\left(\rho_{\alpha}, \rho_{\beta}\right)$ is the LSDA correlation energy density.

Krieger, Chen, Iafrate, and Savin (KCIS) [172] revisited the local density approximation for correlation and attributed the LDA overestimation of correlation energies for nonuniform densities to the fact that, unlike an electron gas, finite many-electron systems have a non-zero energy gap between the Fermi level and the continuum (the gap is equal to the ionization potential [173]). To improve the LDA description of systems with a finite orbital gap, they made use of the formula of Rey and Savin [174] for the correlation energy per particle of the electron gas with an energy gap $G$,

$$
\tilde{\epsilon}_{\mathrm{c}}^{i}(\rho, \nabla \rho)=\frac{\epsilon_{\mathrm{c}}^{i}(\rho, \nabla \rho)+c_{1}^{i}(\rho) G}{1+c_{2}^{i}(\rho) G+c_{3}^{i}(\rho) G^{2}},
$$

where $i=P, F, c_{k}^{i}(\rho)$ are density-dependent coefficients, and $\epsilon_{\mathrm{c}}^{i}(\rho, \nabla \rho)$ is defined in Ref. [175]. They also assumed that the local value of the gap $G$ is determined by the density and its gradient, $G=|\nabla \rho|^{2} / 8 \rho^{2}=\tau_{W} / \rho$, and used the Barth-Hedin-type interpolation formula for $\tilde{\epsilon}_{\mathrm{c}}$, the correlation energy per particle of a spin-polarized electron gas with a gap. The final expression for the KCIS correlation functional was designed to satisfy the same three conditions as those respected by the PBE correlation functional, plus the requirement that the correlation energy vanish for one-electron densities [Eq. (45)]:

$$
E_{\mathrm{c}}^{\mathrm{KCIS}}\left[\rho_{\alpha}, \rho_{\beta}\right]=\int\left[\rho \tilde{\epsilon}_{\mathrm{c}}\left(\rho_{\alpha}, \rho_{\beta}, \nabla \rho_{\alpha}, \nabla \rho_{\beta}\right)-\sum_{\sigma} \frac{\tau_{\sigma}^{W}}{\tau_{\sigma}} \rho_{\sigma} \tilde{\epsilon}_{\mathrm{c}}\left(\rho_{\sigma}, 0, \nabla \rho_{\sigma}, 0\right)\right] d \mathbf{r} .
$$

Perdew, Kurth, Zupan, and Blaha [176] (PKZB) constructed a $\tau$-dependent functional by preserving and extending the list of constraints satisfied by the PBE exchange-correlation GGA. The kinetic energy density appears both in the exchange and correlation components of the PKZB functional. In the exchange part, $\tau$ is an argument of the enhancement factor

$$
F_{\mathrm{x}}^{\mathrm{PKZB}}=1+\frac{\chi}{1+\chi / \kappa},
$$

where $\chi=\chi(\rho, \nabla \rho, \tau)$ is a new variable replacing the variable $\mu s^{2}$ of PBE. The particular form of $\chi$ (not shown here) was chosen to reproduce the fourth-order gradient expansion of $F_{\mathrm{x}}$ for slowly-varying densities. The analytic representation of $\chi$ contains only one empirical parameter optimized by minimizing the mean absolute error in the atomization energies of a 20-molecule training set [176]. 
The PKZB correlation functional combine the ingredients $\epsilon_{\mathrm{c}}^{\mathrm{PBE}}$ and $\tau_{\sigma}$,

$$
\begin{aligned}
E_{\mathrm{c}}^{\mathrm{PKZB}}\left[\rho_{\alpha}, \rho_{\beta}\right]= & \int\left\{\rho \epsilon_{\mathrm{c}}^{\mathrm{PBE}}\left(\rho_{\alpha}, \rho_{\beta}, \nabla \rho_{\alpha}, \nabla \rho_{\beta}\right)\left[1+C\left(\frac{\sum_{\sigma} \tau_{\sigma}^{W}}{\sum_{\sigma} \tau_{\sigma}}\right)^{2}\right]\right. \\
& \left.-(1+C) \sum_{\sigma}\left(\frac{\tau_{\sigma}^{W}}{\tau_{\sigma}}\right)^{2} \rho_{\sigma} \epsilon_{\mathrm{c}}^{\mathrm{PBE}}\left(\rho_{\sigma}, 0, \nabla \rho_{\sigma}, 0\right)\right\} d \mathbf{r},
\end{aligned}
$$

where $C$ is a nonempirical constant chosen to reproduce PBE surface correlation energies of a uniform electron gas. Note that PKZB is self-correlation free for one-electron densities.

PKZB is much more accurate than PBE for atomization energies [177] but inferior for equilibrium bond lengths, vibrational frequencies [177], dissociation energies and, especially, geometries of hydrogen-bonded complexes [178]. These concerns prompted Tao, Perdew, Staroverov, and Scuseria (TPSS) [179] to search for additional constraints to be imposed at the meta-GGA level in order to improve upon PBE and PKZB for all properties. The principal problem with PKZB turned out to be with the exchange component. In PBE and PKZB, the enhancement factors are such that the exchange potentials $v_{\mathrm{x} \sigma}(\mathbf{r})$ diverge at the nuclei (see Fig. 1 of Ref. [180]). In the case of $\mathrm{PBE}$, the divergence of $v_{\mathrm{x} \sigma}(\mathbf{r})$ is harmless, but in the case of PKZB it is manifested in overstretched bond lengths. This problem was addressed in the TPSS approximation by requiring that $v_{\mathrm{x} \sigma}(\mathbf{r})$ be finite at the nucleus whenever $\tau_{\sigma}^{W}=\tau_{\sigma}$, a condition that covers compact iso-orbital densities (i.e., oneand spin-compensated two-electron densities represented by real Kohn-Sham orbitals). In effect, the new constraint eliminates the divergence for nearly all realistic many-electron systems, because $\rho_{\sigma}(\mathbf{r})$ near the nucleus is dominated by the $1 s$ orbital, so that $\tau_{\sigma} \rightarrow \tau_{\sigma}^{W}$. This proved to be the last and key constraint that made it possible to obtain accurate atomization energies and bond lengths from a nonempirical meta-GGA [180].

The TPSS exchange enhancement factor has the same from as in PKZB [Eq. (137)], but the function $\chi(\rho, \nabla \rho, \tau)$ in TPSS is much more complicated (which is necessary to satisfy additional constraints). Aside from producing a finite $v_{\mathrm{x} \sigma}(\mathbf{r})$ at the nucleus, the TPSS exchange functional reproduces the fourth-order density gradient expansion [120] for slowly-varying densities and yields the correct exchange energy for ground-state one-electron hydrogenic densities (for the $\mathrm{H}$ atom, $E_{\mathrm{x}}^{\text {exact }}=-\frac{5}{16} E_{h}$ ). Improvements in the correlation part of TPSS are of a technical character [179]:

$$
E_{\mathrm{c}}^{\mathrm{TPSS}}\left[\rho_{\alpha}, \rho_{\beta}\right]=\int \rho \epsilon_{\mathrm{c}}^{\mathrm{revPKZB}}\left[1+d \epsilon_{\mathrm{c}}^{\mathrm{revPKZB}}\left(\frac{\tau_{W}}{\tau}\right)^{3}\right] d \mathbf{r} .
$$

Here $d$ is a nonempirical constant and $\epsilon_{\mathrm{c}}^{\mathrm{revPKZB}}$ is the revised PKZB correlation energy per particle

$$
\begin{aligned}
\epsilon_{\mathrm{c}}^{\mathrm{revPKZB}} & =\epsilon_{\mathrm{c}}^{\mathrm{PBE}}\left(\rho_{\alpha}, \rho_{\beta}, \nabla \rho_{\alpha}, \nabla \rho_{\beta}\right)\left[1+C(\zeta, \xi)\left(\frac{\tau_{W}}{\tau}\right)^{2}\right] \\
- & {[1+C(\zeta, \xi)]\left(\frac{\tau_{W}}{\tau}\right)^{2} \sum_{\sigma} \frac{\rho_{\sigma}}{\rho} \max \left[\epsilon_{\mathrm{c}}^{\mathrm{PBE}}\left(\rho_{\sigma}, 0, \nabla \rho_{\sigma}, 0\right), \epsilon_{\mathrm{c}}^{\mathrm{PBE}}\left(\rho_{\alpha}, \rho_{\beta}, \nabla \rho_{\alpha}, \nabla \rho_{\beta}\right)\right] }
\end{aligned}
$$

In TPSS, $C$ is no longer a constant but a function of the spin polarization $\zeta$ and a variable $\xi=$ $|\nabla \zeta| / 2\left(3 \pi^{2} \rho\right)^{1 / 3} . C(\zeta, \xi)$ is designed to make $E_{\mathrm{xc}}^{\mathrm{TPSS}}\left[\rho_{\alpha}, \rho_{\beta}\right]$ properly independent of $\zeta$ in the 
low-density limit. The $\max ()$ function in Eq. (140) ensures that $\epsilon_{\mathrm{c}}^{\text {revPKZB }}$ is strictly negative everywhere, a property that is weakly violated by PKZB. Extensive tests on molecules, hydrogen-bonded complexes [180], and solids [181] indicate that, property by property, TPSS overcomes all the shortcomings of PKZB and closely follows or exceeds in accuracy nearly all other density functionals, including B3LYP.

\section{Modeling the exchange-correlation hole}

The sharp cutoff procedure introduces spurious kinks into the GGA exchange hole of Eq. (107), which complicate the derivation of the P86 and PW91 exchange functionals. In this section, we will consider functionals that are based on smooth analytic hole models normalized from the outset.

\subsection{Exchange functionals based on a model hole}

Becke and Roussel [182] constructed a model exchange hole starting with the second-order Taylor expansion of the exact spherically-averaged $\sigma$-spin hole [183]

$$
h_{\mathrm{x}}^{\sigma \sigma}(\mathbf{r}, u)=-\rho_{\sigma}(\mathbf{r})-\frac{1}{6}\left[\nabla^{2} \rho_{\sigma}(\mathbf{r})-4 \tau_{\sigma}(\mathbf{r})+4 \tau_{\sigma}^{W}(\mathbf{r})\right] u^{2}+\ldots
$$

and fitting it to a generalized analytic form of the exact hydrogenic exchange hole. The exact normalized hydrogenic $1 s$ density is $\rho_{\mathrm{H}}(\mathbf{r})=\left(\alpha^{3} / 8 \pi\right) e^{-\alpha|\mathbf{r}|}$, where $\alpha=2 Z$. Hence the spherically-averaged exchange hole for a hydrogenic atom is

$$
\begin{aligned}
h_{\mathrm{x}}^{\mathrm{H}}(\mathbf{r}, u) & =-\frac{1}{4 \pi} \int_{0}^{2 \pi} d \phi_{u} \int_{0}^{\pi} \rho_{\mathrm{H}}(\mathbf{r}+\mathbf{u}) \sin \theta_{u} d \theta_{u} \\
& =-\frac{1}{4 \pi} \int_{0}^{2 \pi} d \phi_{u} \int_{0}^{\pi} \frac{\alpha^{3}}{8 \pi} e^{-\alpha \sqrt{r^{2}+u^{2}-2 r u \cos \theta_{u}}} \sin \theta_{u} d \theta_{u} \\
& =-\frac{\alpha}{16 \pi r u}\left[(\alpha|r-u|+1) e^{-\alpha|r-u|}-(\alpha|r+u|+1) e^{-\alpha|r+u|}\right] .
\end{aligned}
$$

The analytic form of Eq. (142) is sufficiently flexible to parametrize the exact second-order expansion of the exchange hole for any many-electron system, if it is generalized as

$$
h_{\mathrm{x}}^{\mathrm{BR}}(a, b ; u)=-\frac{a}{16 \pi b u}\left[(a|b-u|+1) e^{-a|b-u|}-(a|b+u|+1) e^{-a|b+u|}\right],
$$

where $a$ and $b$ are positive scalar functions of $\rho_{\sigma},\left|\nabla \rho_{\sigma}\right|, \nabla^{2} \rho_{\sigma}$, and $\tau_{\sigma}$, without any physical significance. The underlying hydrogenic model still ensures that the generalized hole of Eq. (143) is nonpositive and normalized to -1 . For a given reference point $\mathbf{r}$, the values of $a(\mathbf{r})$ and $b(\mathbf{r})$ are obtained by expanding Eq. (143) in a Taylor series to second order in $u$ and comparing its zeroth- and second-order coefficients with those of Eq. (141). Then the substitution of Eq. (143) into Eq. (33) yields

$$
E_{\mathrm{x}}^{\mathrm{BR}}\left[\rho_{\alpha}, \rho_{\beta}\right]=-\frac{1}{2} \sum_{\sigma=\alpha, \beta} \int \frac{\rho_{\sigma}(\mathbf{r})}{b(\mathbf{r})}\left[1-e^{-a b}\left(1+\frac{1}{2} a b\right)\right] d \mathbf{r} .
$$


In contrast to the gradient expansion of the exchange hole of Perdew [135], the BR functional does not reduce to LSDA in the uniform density limit. To recover this limit approximately, Becke and Roussel multiplied the term $\left(\tau_{\sigma}-\tau_{\sigma}^{W}\right)$ in Eq. (141) by an adjustment factor of 0.8 . At the same time, the BR exchange energy density has the correct $-\rho(r) / 2 r$ asymptotic behavior in the $r \rightarrow \infty$ limit.

Around the same time, Becke [184] proposed a real-space normalized model for the correlation hole. He started from the observation that, for a given coupling strength $\lambda$, the spin-polarized components of the spherically averaged pair densities have well-defined shortrange behavior near the reference point [185]:

$$
\begin{gathered}
P_{2}^{\sigma \sigma, \lambda}(\mathbf{r}, u)=A^{\sigma \sigma}(\mathbf{r})\left(1+\frac{\lambda}{2} u\right) u^{2}+\ldots, \\
P_{2}^{\alpha \beta, \lambda}(\mathbf{r}, u)=A^{\alpha \beta}(\mathbf{r})(1+\lambda u)+\ldots,
\end{gathered}
$$

where $A^{\sigma \sigma}(\mathbf{r})$ and $A^{\alpha \beta}(\mathbf{r})$ are certain functions. The pair densities trivially determine the exchange-correlation hole by Eq. (10). By using Eqs. (10), (103), the Taylor series expansion for the exchange hole [Eq. (141)], and going through a number of algebraic manipulations, Becke obtained explicit formulas for $h_{\mathrm{c}}^{\sigma \sigma, \lambda}(\mathbf{r}, u)$ and $h_{\mathrm{c}}^{\alpha \beta, \lambda}(\mathbf{r}, u)$. Substitution of these expressions into the adiabatic connection formula of Eq. (29) yields the correlation energy functional

$$
\begin{gathered}
E_{\mathrm{c}}^{\mathrm{B} 88 \mathrm{c}}=E_{\mathrm{c}}^{\alpha \alpha}+E_{\mathrm{c}}^{\beta \beta}+E_{\mathrm{c}}^{\alpha \beta}, \\
E_{\mathrm{c}}^{\sigma \sigma}=C_{\sigma \sigma} \int \rho_{\sigma}\left(\tau_{\sigma}-\tau_{\sigma}^{W}\right) z_{\sigma \sigma}^{4}\left[1-\frac{2}{z_{\sigma \sigma}} \ln \left(1+\frac{z_{\sigma \sigma}}{2}\right)\right] d \mathbf{r}, \\
E_{\mathrm{c}}^{\alpha \beta}=C_{\alpha \beta} \int \rho_{\alpha} \rho_{\beta} z_{\alpha \beta}^{2}\left[1-\frac{1}{z_{\alpha \beta}} \ln \left(1+z_{\alpha \beta}\right)\right] d \mathbf{r},
\end{gathered}
$$

where $z_{\sigma \sigma}$ and $z_{\alpha \beta}$ are the so-called correlation lengths, defined in Ref. [184], while $C_{\sigma \sigma}$ and $C_{\alpha \beta}$ are adjustable constants.

Performance of the BR and B88c functionals has been studied by Becke [186], as well as by Neumann and Handy [187]. A comprehensive assessment of these functionals, completed recently by Izmaylov et al. [188], indicates that the accuracy of $\mathrm{BR}$ is comparable to that of the B88 exchange, while the B88c approximation is less accurate than LYP, PW91, and similar correlation functionals.

Approximate functionals derived from a model exchange-correlation hole are relatively few in number. The majority of functionals are not based on any explicit model hole. The holes corresponding to such functionals are not even known and, if needed, have to be "reverse-engineered" from the functional itself. Ernzerhof and Perdew [189] did this to obtain a smooth analytic representation for the angle- and system-averaged PBE GGA exchange hole. When substituted into Eq. (33), their model gives the hole-based PBE functional [189]. For practical purposes, the hole-based PBE exchange is numerically equivalent to the energybased functional of Eq. (126). Explicit exchange-correlation holes are of considerable interest on their own. The work on the TPSS hole is currently in progress [190]. 


\subsection{Functionals based on a correlated wave function}

The best-known example of approximations of this type is the correlation functional of Lee, Yang, and Parr (LYP) [191]. LYP is nothing but a DFT adaptation of the orbitaldependent correlation energy formula of Colle and Salvetti (CS) [192]. The starting point of the derivation is a correlated wave function of the form

$$
\Psi\left(\mathbf{x}_{1}, \mathbf{x}_{2}, \ldots, \mathbf{x}_{N}\right)=\Psi^{\mathrm{HF}}\left(\mathbf{x}_{1}, \mathbf{x}_{2}, \ldots, \mathbf{x}_{N}\right) \prod_{i<j}\left[1-f\left(\mathbf{r}_{i}, \mathbf{r}_{j}\right)\right]
$$

where $\Psi^{\mathrm{HF}}$ is the Hartree-Fock wave function (a single determinant) and $f\left(\mathbf{r}_{i}, \mathbf{r}_{j}\right)$ is a model two-electron correlation function. Arguing that $f\left(\mathbf{r}_{i}, \mathbf{r}_{j}\right)$ is small for typical positions of electrons, Colle and Salvetti obtained an approximate formula for the corresponding electron pair density

$$
P_{2}^{\mathrm{CS}}\left(\mathbf{r}_{1}, \mathbf{r}_{2}\right)=P_{2}^{\mathrm{HF}}\left(\mathbf{r}_{1}, \mathbf{r}_{2}\right)\left[1-f\left(\mathbf{r}_{1}, \mathbf{r}_{2}\right)\right]^{2},
$$

where $P_{2}^{\mathrm{HF}}\left(\mathbf{r}_{1}, \mathbf{r}_{2}\right)$ is the spin-free Hartree-Fock electron pair density of Eq. (37). The conventional quantum-mechanical correlation energy is then given by

$$
\begin{aligned}
E_{\mathrm{c}}^{\mathrm{CS}} & =\iint \frac{P_{2}^{\mathrm{CS}}\left(\mathbf{r}_{1}, \mathbf{r}_{2}\right)-P_{2}^{\mathrm{HF}}\left(\mathbf{r}_{1}, \mathbf{r}_{2}\right)}{r_{12}} d \mathbf{r}_{1} d \mathbf{r}_{2} \\
& =\iint P_{2}^{\mathrm{HF}}\left(\mathbf{r}_{1}, \mathbf{r}_{2}\right) \frac{f^{2}\left(\mathbf{r}_{1}, \mathbf{r}_{2}\right)-2 f\left(\mathbf{r}_{1}, \mathbf{r}_{2}\right)}{r_{12}} d \mathbf{r}_{1} d \mathbf{r}_{2} .
\end{aligned}
$$

By making a series of approximations, Colle and Salvetti then obtained the following expression in terms of interparticle coordinates $\mathbf{R}=\left(\mathbf{r}_{1}+\mathbf{r}_{2}\right) / 2$ and $\mathbf{u}=\mathbf{r}_{1}-\mathbf{r}_{2}$

$$
E_{\mathrm{c}}^{\mathrm{CS}}=-a \int \rho(\mathbf{R}) \frac{1+b \rho^{-8 / 3}(\mathbf{R})\left[\nabla_{\mathbf{u}}^{2} P_{2}^{\mathrm{HF}}(\mathbf{R}, \mathbf{u})\right]_{u=0} \exp \left[-c \rho^{-1 / 3}(\mathbf{R})\right]}{1+d \rho^{-1 / 3}(\mathbf{R})} d \mathbf{R}
$$

where the values of the parameters $a=0.04918, b=0.132, c=0.2533$, and $d=0.349$ were determined by fitting to certain exact data relevant to the He atom.

Lee, Yang, and Parr [191] carried out this analysis further by rewriting Eq. (153) in an equivalent form

$$
E_{\mathrm{c}}^{\mathrm{CS}}[\rho]=-a \int \rho(\mathbf{r}) \frac{1+b \rho^{-5 / 3}(\mathbf{r})\left[\tau_{\mathrm{HF}}(\mathbf{r})-2 \tilde{\tau}_{W}(\mathbf{r})\right] \exp \left[-c \rho^{-1 / 3}(\mathbf{r})\right]}{1+d \rho^{-1 / 3}(\mathbf{r})} d \mathbf{r},
$$

where

$$
\tilde{\tau}_{W}=\frac{1}{8} \frac{|\nabla \rho|^{2}}{\rho}-\frac{1}{8} \nabla^{2} \rho
$$

and $\tau_{\mathrm{HF}}$ is the Hartree-Fock kinetic energy density [note also the addition of the Laplacian term which was not included in the definition of $\tau_{W}$ in Eq. (127)]. Eq. (154) is still not a conventional density functional, because it involves dependence on the orbitals through the $\tau_{\mathrm{HF}}$ term. Lee, Yang, and Parr simplified this expression by replacing $\tau_{\mathrm{HF}}$ with its second-order gradient expansion

$$
\tau_{\mathrm{HF}} \approx \tau_{\mathrm{unif}}+\frac{1}{9} \tilde{\tau}_{W}+\frac{1}{18} \nabla^{2} \rho
$$


where $\tau_{\text {unif }}=\frac{3}{10}\left(3 \pi^{2}\right)^{2 / 3} \rho^{5 / 3}$ is the Thomas-Fermi kinetic energy density of a uniform electron gas. The final expression,

$$
E_{\mathrm{c}}^{\mathrm{LYP}}[\rho]=-a \int \frac{\rho+b \rho^{-2 / 3}\left(C_{F} \rho^{5 / 3}-2 \tilde{\tau}_{W}+\frac{1}{9} \tilde{\tau}_{W}+\frac{1}{18} \nabla^{2} \rho\right) \exp \left(-c \rho^{-1 / 3}\right)}{1+d \rho^{-1 / 3}} d \mathbf{r},
$$

where $C_{F}=\frac{3}{10}\left(3 \pi^{2}\right)^{2 / 3}$, proved to be an excellent approximation to Eq. (153).

The LYP functional as given by Eq. (157) is a meta-GGA, because it depends on the Laplacian of the density. Miehlich et al. [193] pointed out that $\nabla^{2} \rho(\mathbf{r})$ can be eliminated from the LYP formula by partial integration. Their transformed version of LYP, reproduced here in the spin-polarized form, is given by [194]

$$
\begin{aligned}
E_{\mathrm{c}}^{\mathrm{LYP}}\left[\rho_{\alpha}, \rho_{\beta}\right]= & -a \int\left[\frac{4}{1+d \rho^{-1 / 3}} \frac{\rho_{\alpha} \rho_{\beta}}{\rho}+2{ }^{11 / 3} C_{F} b \omega \rho_{\alpha} \rho_{\beta}\left(\rho_{\alpha}^{8 / 3}+\rho_{\beta}^{8 / 3}\right)\right. \\
& \left.+L_{\alpha \alpha}\left|\nabla \rho_{\alpha}\right|^{2}+L_{\alpha \beta} \nabla \rho_{\alpha} \cdot \nabla \rho_{\beta}+L_{\beta \beta}\left|\nabla \rho_{\beta}\right|^{2}\right] d \mathbf{r},
\end{aligned}
$$

where

$$
\begin{gathered}
L_{\alpha \alpha}=b \omega\left\{\frac{1}{9} \rho_{\alpha} \rho_{\beta}\left[1-3 \delta-(\delta-11) \frac{\rho_{\alpha}}{\rho}\right]-\rho_{\beta}^{2}\right\}, \\
L_{\alpha \beta}=b \omega\left[\frac{1}{9} \rho_{\alpha} \rho_{\beta}(47-7 \delta)-\frac{4}{3} \rho^{2}\right],
\end{gathered}
$$

$L_{\beta \beta}$ is obtained by interchanging subscripts $\alpha$ and $\beta$ in Eq. (159),

$$
\omega=\frac{e^{-c \rho^{-1 / 3}}}{1+d \rho^{-1 / 3}} \rho^{-11 / 3}, \quad \delta=c \rho^{-1 / 3}+\frac{d \rho^{-1 / 3}}{1+d \rho^{-1 / 3}},
$$

and $a, b, c, d$ are the same parameters as in Eq. (153).

Although the LYP functional does not reduce to LSDA for uniform densities (only about $25 \%$ of the true correlation energy of a uniform electron gas is recovered [195]), it is an accurate approximation for atomic correlation energies. The good performance of LYP, however, appears to be a fluke. In a critical analysis, Singh, Massa, and Sahni [196] demonstrated that the Colle-Salvetti correlation energy formula is seriously flawed. For example, the wave function of Eq. (150) is not normalized, and the Kohn-Sham correlation potential corresponding to the Colle-Salvetti formula is grossly inaccurate [197]. Their analysis was continued by Imamura, Scuseria, and Martin [198].

The idea of generating density functionals from correlated wave functions continues to attract attention [199]. Imamura and Scuseria [200] recently derived a correlation functional starting from a Colle-Salvetti type correlated wave function and using the transcorrelated method of Boys and Handy [201, 202]. Colle-Salvetti-type correlation functionals that treat parallel-spin and opposite-spin contributions to the correlation energy separately have been also developed by Tsuneda and Hirao [203], Tsuneda, Suzumura, and Hirao [204].

\subsection{Functionals based on a model pair correlation function}

The exchange-correlation hole $h_{\mathrm{xc}}^{\lambda}\left(\mathbf{r}_{1}, \mathbf{r}_{2}\right)$ is closely associated with the pair distribution function $g^{\lambda}\left(\mathbf{r}_{1}, \mathbf{r}_{2}\right)$ defined by

$$
P_{2}^{\lambda}\left(\mathbf{r}_{1}, \mathbf{r}_{2}\right)=\frac{1}{2} \rho\left(\mathbf{r}_{1}\right) \rho\left(\mathbf{r}_{2}\right) g^{\lambda}\left(\mathbf{r}_{1}, \mathbf{r}_{2}\right)
$$


where $P_{2}^{\lambda}$ is the pair density at coupling strength $\lambda$. Comparison with Eq. (10) shows that

$$
h_{\mathrm{xc}}^{\lambda}\left(\mathbf{r}_{1}, \mathbf{r}_{2}\right)=\rho\left(\mathbf{r}_{2}\right)\left[g^{\lambda}\left(\mathbf{r}_{1}, \mathbf{r}_{2}\right)-1\right] .
$$

Note that $g^{\lambda}\left(\mathbf{r}_{1}, \mathbf{r}_{2}\right)$ is symmetric in its variables, unlike $h_{\mathrm{xc}}^{\lambda}\left(\mathbf{r}_{1}, \mathbf{r}_{2}\right)$. At $\lambda=0, g^{\lambda}=g_{\mathrm{x}}+g_{\mathrm{c}}^{\lambda}$ reduces to the exchange-only pair-distribution function $g_{\mathrm{x}}$. This implies that $h_{\mathrm{x}}\left(\mathbf{r}_{1}, \mathbf{r}_{2}\right)=$ $\rho\left(\mathbf{r}_{2}\right)\left[g_{\mathrm{x}}\left(\mathbf{r}_{1}, \mathbf{r}_{2}\right)-1\right]$ and $h_{\mathrm{c}}^{\lambda}\left(\mathbf{r}_{1}, \mathbf{r}_{2}\right)=\rho\left(\mathbf{r}_{2}\right) g_{\mathrm{c}}^{\lambda}\left(\mathbf{r}_{1}, \mathbf{r}_{2}\right)$. It is easy to establish that the pair distribution function determines the exchange-correlation energy by a formula similar to Eq. (31), and that

$$
E_{\mathrm{c}}=\frac{1}{2} \int_{0}^{1} d \lambda \iint \rho\left(\mathbf{r}_{1}\right) \rho\left(\mathbf{r}_{2}\right) \frac{g_{\mathrm{c}}^{\lambda}\left(\mathbf{r}_{1}, \mathbf{r}_{2}\right)}{r_{12}} d \mathbf{r}_{1} d \mathbf{r}_{2} .
$$

Although $g_{\mathrm{c}}^{\lambda}\left(\mathbf{r}_{1}, \mathbf{r}_{2}\right)$ is trivially related to $h_{\mathrm{c}}^{\lambda}\left(\mathbf{r}_{1}, \mathbf{r}_{2}\right)$, it is instructive to treat density functionals based on $g_{\mathrm{c}}^{\lambda}\left(\mathbf{r}_{1}, \mathbf{r}_{2}\right)$ as a separate subgroup. The function $g_{\mathrm{c}}^{\lambda}\left(\mathbf{r}_{1}, \mathbf{r}_{2}\right)$ is either derived (approximately) from a correlated wave function (the Colle-Salvetti formula can be viewed in this light) or postulated as a "model".

Proynov, Salahub, and coworkers have developed several correlation functionals [205-209] starting from a Gaussian model of the spherically-averaged pair distribution function for opposite-spin electrons

$$
g_{\mathrm{c}, \alpha \beta}^{\lambda}(\mathbf{R}, u)=-e^{-z_{\alpha \beta}^{2} u^{2}}\left[F_{1}(\mathbf{R}, \lambda)+F_{2}(\mathbf{R}, \lambda)\right],
$$

where $F_{1}(\mathbf{R}, \lambda)$ and $F_{2}(\mathbf{R}, \lambda)$ are certain parametrized functions and $z_{\alpha \beta}$ is the correlation length. Substitution of $g_{\mathrm{c}, \alpha \beta}^{\lambda}$ into Eq. (164), followed by analytic integration over $\lambda$, yields

$$
E_{\mathrm{c}}^{\alpha \beta}=\frac{1}{2} \int d \mathbf{R} \int_{0}^{\infty}\left\langle\rho_{\alpha}(\mathbf{R}+\mathbf{u} / 2) \rho_{\beta}(\mathbf{R}-\mathbf{u} / 2)\right\rangle \bar{g}_{\mathrm{c}, \alpha \beta}(\mathbf{R}, u) u d u
$$

where $\langle\ldots\rangle$ indicates averaging over the spherical components of $\mathbf{u}$. In the LAP1 and LAP2 models [206], the opposite-spin contribution to the correlation energy $\left(E_{\mathrm{c}}^{\alpha \beta}\right)$ is obtained by Eq. (166), the $E_{\mathrm{c}}^{\sigma \sigma}$ terms are neglected, and the total correlation energy is approximated as $E_{\mathrm{c}}=2 E_{\mathrm{c}}^{\alpha \beta}$. The LAP3 model [208] takes into account the parallel spin contributions and approximates them by the formula $\epsilon_{\mathrm{c}}^{\sigma \sigma}=\left(1-1 / N_{\sigma}\right) C_{p} \epsilon_{\mathrm{c}}^{\sigma \sigma^{\prime}}$, where $N_{\sigma}$ is the number of spin- $\sigma$ electrons and $C_{p}$ is an empirical factor. The total correlation energy is then given as the sum of these components. The spherical average in the LAP1-LAP3 models is approximated by the first term of the Taylor series expansion about $\mathbf{R}$ :

$$
\left\langle\rho_{\alpha}(\mathbf{R}+\mathbf{u} / 2) \rho_{\beta}(\mathbf{R}-\mathbf{u} / 2)\right\rangle \approx 4 \pi \rho_{\alpha}(\mathbf{R}) \rho_{\beta}(\mathbf{R}),
$$

but in a recent revision [209], called the $\tau 1$-model, the right-hand side of Eq. (167) is augmented by a correction term that depends on $\rho,|\nabla \rho|$, and $\nabla^{2} \rho$.

\subsection{Functionals based on a density matrix expansion}

The exact exchange energy of a closed-shell system can be obtained from the density matrix $\rho\left(\mathbf{r}_{1}, \mathbf{r}_{2}\right)$ by Eq. (38). Replacing $\rho\left(\mathbf{r}_{1}, \mathbf{r}_{2}\right)$ in this formula by a truncated expansion in $\rho$ and its gradients is a natural path to an exchange functional. This idea was pioneered by Negele 
and Vautherin [210] in the framework of nuclear matter theory and developed into practical exchange functionals for quantum chemistry by Scuseria and coworkers [211-214]. Originally, Negele and Vautherin expanded $\rho\left(\mathbf{r}_{1}, \mathbf{r}_{2}\right)$ in transformed coordinates $\mathbf{R}=\left(\mathbf{r}_{1}+\mathbf{r}_{2}\right) / 2$ and $\mathbf{u}=\mathbf{r}_{1}-\mathbf{r}_{2}$. Koehl et al. [211] generalized this transformation to

$$
\mathbf{R}=a \mathbf{r}_{1}+(1-a) \mathbf{r}_{2}, \quad \mathbf{u}=\mathbf{r}_{1}-\mathbf{r}_{2},
$$

where $0 \leq a \leq \frac{1}{2}$. After performing the Taylor expansion of $\rho(\mathbf{R}, \mathbf{u})$ around $\mathbf{u}=0$, averaging the series over the angular coordinates of $\mathbf{u}$, expanding the result in Bessel functions $j_{n}(u)$ and Legendre polynomials $P_{n}(\mathbf{R})$, and truncating the series after the second-order terms, one obtains [211]

$$
\rho(\mathbf{R}, u)=\frac{3 j_{1}(k u)}{k u} \rho(\mathbf{R})+\frac{35 j_{3}(k u)}{2 k^{3} u}\left[\left(a^{2}-a+\frac{1}{2}\right) \nabla^{2} \rho(\mathbf{R})-2 \tau(\mathbf{R})+\frac{3}{5} k^{2} \rho(\mathbf{R})\right],
$$

where $k$ is the relative momentum of two electrons. Substitution of this density matrix expansion (DME) into Eq. (38) gives

$$
E_{\mathrm{x}}^{\mathrm{DME}}[\rho]=-\pi \int\left\{\frac{9}{4 k^{2}} \rho^{2}+\frac{35}{12 k^{4}} \rho\left[\left(a^{2}-a+\frac{1}{2}\right) \nabla^{2} \rho-2 \tau+\frac{3}{5} k^{2} \rho\right]\right\} d \mathbf{r} .
$$

Note that the choice $k=k_{F}$, regardless of the value of $a$, yields the LDA (Dirac) exchange formula (58) as the first term of $E_{\mathrm{x}}^{\mathrm{DME}}[\rho]$. The remaining terms containing $\nabla \rho, \nabla^{2} \rho$, and $\tau$, naturally arise as corrections to the LDA.

Surprisingly, the nonempirical functional of Eq. (170) with $a=0$ (the implicit choice of Negele and Vautherin) proved to be a worse approximation than LDA, with a mean absolute error (m.a.e.) of $3.66 E_{h}$ for a test set of 32 molecules, compared to $1.79 E_{h}$ for LDA. Koehl, Odom, and Scuseria (KOS) suggested treating $a$ as an adjustable parameter and found that at its optimal value $(a=0.0000638)$ the error of Eq. (170) drops to $0.087 E_{h}$, which is only about twice as large as the error of B88 (m.a.e. $=0.041 E_{h}$ ). By setting $a=0$ and optimizing the coefficients of the $\rho^{2}$ and $\rho$ terms they found

$$
E_{\mathrm{x}}^{\mathrm{KOS}}[\rho]=\int\left[\frac{A}{k_{F}^{2}} \rho^{2}+\frac{B}{k_{F}^{4}} \rho\left(\frac{1}{2} \nabla^{2} \rho-2 \tau+\frac{3}{5} k_{F}^{2} \rho\right)\right] d \mathbf{r},
$$

where $A=-7.31275$ and $B=-5.43182$. The m.a.e. of the KOS functional for the same 32-molecule test set is only $0.026 E_{h}$.

Recently, Maximoff and Scuseria [214] worked out the full fourth-order density matrix expansion (DME4) and proposed a corresponding exchange functional called DME4 $x$. In test calculations, DME4x produced some of the most accurate atomic and molecular exchange energies. Developing exchange functionals by incorporating higher orders of the density matrix expansion may look like a safe bet, but in practice this approach is wrought with difficulties. High-order density matrix expansions involve unusual ingredients like $\nabla^{n} \rho(\mathbf{r})$ and $\nabla^{n} \tau(\mathbf{r})$ with $n>1$, whose presence causes an extreme numerical sensitivity of exchangecorrelation matrix elements and makes self-consistent calculations difficult to converge.

\section{$9 \quad$ Empirical fits}

A pragmatic approach to developing density functional approximations is to expand the post-LDA correction in a set of suitably chosen parametrized functions and optimize the 
parameters by training the functional to reproduce certain calibration data as accurately as possible. Of course, fitted parameters appear in many of the previously discussed functionals, but there they were used only to "clean up" the construction. In this survey, we call "empirical" or "optimized" only those functionals whose design is avowedly empirical.

Optimization of empirical parameters is often accompanied by relaxing exact constraints. As a result, many optimized functionals violate some of the basic analytic properties, such as the uniform density limit of Eq. (46). For instance, Slater's $X \alpha$ method [9-11], which is nowadays regarded as an empirical variant of Dirac's exchange, clearly does so.

Systematic procedures for optimizing GGA and meta-GGA exchange-correlation functionals have been developed by Becke [215, 216], Adamson et al. [217], Handy and coworkers [218-221]. In Becke's method, the total exchange-correlation energy is written as

$$
E_{\mathrm{xc}}=\sum_{\sigma} E_{\mathrm{x}}^{\sigma}+E_{\mathrm{c}}^{\alpha \beta}+\sum_{\sigma} E_{\mathrm{c}}^{\sigma \sigma}
$$

where the individual energy components have the form

$$
\begin{gathered}
E_{\mathrm{x}}^{\sigma}=\int e_{\mathrm{x} \sigma}^{\mathrm{UEG}}\left(\rho_{\sigma}\right) F_{\mathrm{x}}^{\sigma} d \mathbf{r}, \\
E_{\mathrm{c}}^{\alpha \beta}=\int e_{\mathrm{c} \alpha \beta}^{\mathrm{UEG}}\left(\rho_{\alpha}, \rho_{\beta}\right) F_{\mathrm{c}}^{\alpha \beta} d \mathbf{r}, \\
E_{\mathrm{c}}^{\sigma \sigma}=\int e_{\mathrm{c} \sigma \sigma}^{\mathrm{UEG}}\left(\rho_{\sigma}\right) \eta_{\sigma}^{\mathrm{SCC}} F_{\mathrm{c}}^{\sigma \sigma} d \mathbf{r} .
\end{gathered}
$$

Here $e_{\mathrm{x} \sigma}^{\mathrm{UEG}}\left(\rho_{\sigma}\right)=-2^{1 / 3} C_{\mathrm{x}} \rho_{\sigma}^{4 / 3}, e_{\mathrm{c}}^{\mathrm{UEG}}$ are the correlation energy densities of a uniform electron gas given by Eqs. (133) and (134); $F$ are enhancement factors (functions of $\rho_{\sigma}, \nabla \rho_{\sigma}$ and, possibly, $\nabla^{2} \rho_{\sigma}$ and $\tau_{\sigma}$ ), and $\eta_{\sigma}^{\mathrm{SCC}}$ is the self-correlation correction of Eq. (129). Each $F$ is represented by a polynomial

$$
F=\sum_{m=0}^{n} c_{m} w^{m}\left(\rho_{\sigma}, \nabla \rho_{\sigma}, \ldots\right),
$$

where $w$ is a suitably chosen function derived from approximate expressions for gradient corrections [215]; $c_{m}$ are empirical coefficients determined by least-squares fitting to training sets of accurate experimental and/or theoretical data. Eqs. (172)-(176) underly several empirical functionals, such as Becke's 1997 (B97) exchange-correlation [215], Schmider-Becke's 1998 hybrid GGA (SB98h) [222] and hybrid meta-GGA ( $\tau$-SB98h) [223], the GGA of Hamprecht, Cohen, Tozer, and Handy (HCTH) [219], its various reparametrizations (HCTH/120, $\mathrm{HCTH} / 147$ [224], and HCTH/407 [225]), and $\tau$-HCTH [226].

In 1997, Van Voorhis and Scuseria [212] argued that, since electron densities in chemistry are not uniform, one may generalize the density matrix expansion of Eq. (169) by replacing the fixed $k=k_{F}$ by a variable $k^{2} \rightarrow k_{F}^{2} w(x, z)$, where $w(x, z)$ is a function of $x=|\nabla \rho| / \rho^{4 / 3}$ and $z=2\left(\tau / \rho^{5 / 3}-C_{F}\right)$. Accordingly, they proposed two exchange functionals of the form

$$
F_{\mathrm{x}}^{\mathrm{VS} 97}(x, z)=a+\frac{b_{1} x^{2}+b_{2} z}{w(x, z)}
$$


where $w(x, z)=1+\alpha_{1} x^{2}+\alpha_{2} z$, and $a, b_{1}, b_{2}, \alpha_{1}, \alpha_{2}$ are empirical parameters. The resulting approximations were found to be in a better agreement with the Hartree-Fock values than most of the other exchange functionals available at the time.

In 1998, Van Voorhis and Scuseria extended this approach [213] to include more terms:

$$
F_{\mathrm{x}}^{\mathrm{VS} 98}(x, z)=\frac{a}{w(x, z)}+\frac{b_{1} x^{2}+b_{2} z}{w^{2}(x, z)}+\frac{c_{1} x^{4}+c_{2} x^{2} z+c_{3} z^{2}}{w^{3}(x, z)},
$$

where $a, b_{i}$, and $c_{i}$ are empirical parameters. The form of the function $w(x, z)=1+\alpha\left(x^{2}+z\right)$ was chosen to satisfy certain nonuniform scaling relations. Notice that neither VS97 nor VS98 reduces to the LSDA for uniform densities.

For the VS98 correlation, Van Voorhis and Scuseria [213] assumed the general form of Eq. (174) and (175) with the Perdew-Wang parametrization [114] of $\epsilon_{\mathrm{c}}^{\mathrm{LSDA}}\left(\rho_{\alpha}, \rho_{\beta}\right)$. Hoping for cancellation of errors, they chose the factors $F_{\mathrm{c}}^{\alpha \beta}(x, z)$ and $F_{\mathrm{c}}^{\sigma \sigma}(x, z)$ to have the same analytic form as the exchange enhancement factor of Eq. (178). The VS98 exchangecorrelation functional (also referred to as VSXC, after the keyword in GAUSSIAN) has 21 empirical parameters (the exchange component, the parallel-, and opposite-spin correlation components have 7 parameters each), and is the most accurate functional in existence for atomization energies, surpassing even the hybrid functionals (see Table 2 below). Also, because the parameters of VS98 were optimized for exchange and correlation together, neither component is accurate separately. Nevertheless, the analytic form of Eq. (178) is very flexible, so that a suitable reparametrization of the stand-alone VS98 exchange functional can produce excellent approximations to the exact exchange energies [213].

Another approach is to optimize linear combinations of several existing functionals,

$$
E_{\mathrm{xc}}=\sum_{k} c_{k} E_{\mathrm{xc}}^{(k)}
$$

possibly with a focus on a particular property. Internal parameters of individual functionals $E_{\mathrm{xc}}^{(k)}$ may also be subject to reoptimization. This type of approximations is represented by EDF1 ("empirical density functional 1") of Adamson, Gill, and Pople [217], which was optimized to yield accurate thermochemistry, and EDF2 of Lin, George, and Gill, optimized to give accurate vibrational frequencies [227].

In search for a functional that would accurately reproduce the Hartree-Fock energies of first and second-row atoms, Handy and Cohen [228] refined Becke's 1986 exchange approximation [Eq. (91)] by optimizing the coefficient of the LSDA term and revising the gradient correction. The enhancement factor of their functional, called OPTX, is given by

$$
F_{\mathrm{x}}^{\mathrm{OPTX}}(s)=a_{1}+a_{2} c_{2}\left[\frac{\gamma\left(c_{1} s\right)^{2}}{1+\gamma\left(c_{1} s\right)^{2}}\right]^{2},
$$

where $s$ is the reduced gradient of Eq. (79), $a_{1}=1.05151, a_{2}=1.43169, \gamma=0.006$ are empirical parameters, and $c_{1}=2\left(6 \pi^{2}\right)^{1 / 3}$ and $c_{2}=\left(2^{1 / 3} C_{\mathrm{x}}\right)^{-1}$. Tests of the OPTX functional paired with LYP indicate [229] that the OLYP model is perceptibly more accurate than BLYP, although not as accurate [180] as meta-GGAs or hybrid functionals (see also Table 2). Several reparametrizations of standard functionals like the B97 GGA and the VS98 metaGGA have been discussed in Ref. [230]. 
$\mathrm{Xu}$ and Goddard (XG) [231] proposed an exchange functional of the form

$$
F_{\mathrm{x}}^{\mathrm{XG}}(s)=1+a_{1}\left[F_{\mathrm{x}}^{\mathrm{B} 88}(s)-1\right]+a_{2}\left[F_{\mathrm{x}}^{\mathrm{PW} 91}(s)-1\right],
$$

where the mixing parameters $a_{1}=0.722$ and $a_{2}=0.347$ were determined by least squares fitting to the total energy of 10 atoms and atomization energies of 38 molecules. In combination with the LYP correlation, the XG exchange functional is reported to show superior performance for transition metals, hydrogen- and van der Waals complexes.

Purely empirical functionals often work well, because satisfaction of many secondary constraints (the uniform, high- and low-density limits, density scaling transformations, and so on) is not essential for a good performance for usual properties ("molecules do not behave like the uniform electron gas"). The price for avoiding the hard work of constraint satisfaction is that optimized functionals may fail badly in situations for which they were not "trained".

\section{Mixing exact and approximate exchange}

Functionals that combine GGAs or meta-GGAs with exact exchange of Eq. (38) are called hybrids. At present, hybrid functionals outnumber any other group of exchange-correlation approximations. "Hybridization" has been embraced widely because it greatly improves performance of "pure DFT" functionals and is easy to implement.

\subsection{Global hybrids}

The idea of mixing density functional approximations with exact (Hartree-Fock-like) exchange rests on theoretical considerations involving the adiabatic connection formula [Eq. (28)]. Becke [232] reasoned that, since $E_{\mathrm{xc}}^{\lambda=0}=E_{\mathrm{x}}^{\text {exact }}$ and $E_{\mathrm{xc}}^{\lambda=1} \approx E_{\mathrm{xc}}^{\mathrm{LSDA}}$, the integral over $\lambda$ in Eq. (28) can be approximated by the mean value theorem:

$$
E_{\mathrm{xc}} \approx \frac{1}{2}\left(E_{\mathrm{x}}^{\mathrm{exact}}+E_{\mathrm{xc}}^{\mathrm{LSDA}}\right) .
$$

Eq. (182) has been termed the "half-and-half" theory [232]. The extent and validity of this approximation were analyzed in detail by Levy, March, and Handy [233], as well as Proynov et al. [207].

Soon thereafter, Becke proposed [234] a more empirical yet much more accurate threeparameter hybrid model

$$
E_{\mathrm{xc}}=a_{0} E_{\mathrm{x}}^{\mathrm{exact}}+\left(1-a_{0}\right) E_{\mathrm{x}}^{\mathrm{LSDA}}+a_{\mathrm{x}} \Delta E_{\mathrm{x}}^{\mathrm{GGA}}+E_{\mathrm{c}}^{\mathrm{LSDA}}+a_{\mathrm{c}} \Delta E_{\mathrm{c}}^{\mathrm{GGA}},
$$

where $\Delta E_{\mathrm{x}}^{\mathrm{GGA}}$ and $\Delta E_{\mathrm{c}}^{\mathrm{GGA}}$ are gradient correction parts of the GGA exchange and correlation functionals, and $a_{0}, a_{\mathrm{x}}$, and $a_{\mathrm{c}}$ are adjustable parameters. The original three-parameter hybrid functional of Becke [234] has the form

$$
E_{\mathrm{xc}}^{\mathrm{B} 3 \mathrm{PW} 91}=a_{0} E_{\mathrm{x}}^{\mathrm{exact}}+\left(1-a_{0}\right) E_{\mathrm{x}}^{\mathrm{LSDA}}+a_{\mathrm{x}} \Delta E_{\mathrm{x}}^{\mathrm{B} 88}+E_{\mathrm{c}}^{\mathrm{LSDA}(\mathrm{PW} 92)}+a_{\mathrm{c}} \Delta E_{\mathrm{c}}^{\mathrm{PW} 91},
$$

where $\Delta E_{\mathrm{x}}^{\mathrm{B} 88}$ is the beyond-LSDA part of the B88 exchange, $E_{\mathrm{c}}^{\mathrm{LSDA}(\mathrm{PW} 92)}$ is the PerdewWang parametrization [114] of the LSDA correlation, $\Delta E_{\mathrm{c}}^{\mathrm{PW} 91}$ is the gradient correction of 
Table 1: Parameters (in $E_{h}$ ) of the VWN and VWN-RPA correlation functionals [113] of Eq. (73). The value of $A_{\mathrm{GB}}$ is given by Eq. (70).

\begin{tabular}{|c|c|c|c|c|c|}
\hline \multirow[b]{2}{*}{ Parameter } & \multicolumn{3}{|c|}{ VWN } & \multicolumn{2}{|c|}{ VWN-RPA } \\
\hline & $\epsilon_{\mathrm{c}}^{P}\left(r_{s}\right)$ & $\epsilon_{\mathrm{c}}^{F}\left(r_{s}\right)$ & $-\alpha_{\mathrm{c}}\left(r_{s}\right)$ & $\epsilon_{\mathrm{c}}^{P}\left(r_{s}\right)$ & $\epsilon_{\mathrm{c}}^{F^{\prime}}\left(r_{s}\right)$ \\
\hline$A$ & $A_{\mathrm{GB}}$ & $A_{\mathrm{GB}} / 2$ & $1 / 6 \pi^{2}$ & $A_{\mathrm{GB}}$ & $A_{\mathrm{GB}} / 2$ \\
\hline$x_{0}$ & -0.10498 & -0.32500 & -0.00475840 & -0.409286 & -0.743294 \\
\hline$b$ & 3.72744 & 7.06042 & 1.13107 & 13.0720 & 20.1231 \\
\hline$c$ & 12.9352 & 18.0578 & 13.0045 & 42.7198 & 101.578 \\
\hline
\end{tabular}

the PW91 correlation [the second term on the right-hand side of Eq. (114)], and $a_{0}, a_{\mathrm{x}}$, and $a_{\mathrm{c}}$ are empirical constants. The optimal values of these constants were determined by a fit to a series of atomization energies, ionization potentials, and proton affinities to give $a_{0}=0.20$, $a_{\mathrm{x}}=0.72$, and $a_{\mathrm{c}}=0.81[234]$.

Using Eq. (184) as a template, the developers of the GAUSSIAN program [32] introduced two other well-known three-parameter hybrid functionals, B3LYP and B3P86. Unfortunately, these two functionals are scantily documented in the literature. The original paper by Becke [234] contains no mention of either B3LYP or B3P86. B3LYP debuted very inconspicuously in a paper titled "Ab initio calculation of vibrational absorption and circular dichroism spectra using density functional force fields" [235], while B3P86 is defined solely by its implementation in the GAUSSIAN program. To complicate the matter, the B3LYP functional is coded in GAUSSIAN not exactly as intended in Ref. [235]. We would like to use this opportunity to clarify several issues concerning these functionals.

The B3LYP hybrid, as implemented in the GAUSSIAN program, is given by

$$
E_{\mathrm{xc}}^{\mathrm{B} 3 \mathrm{LYP}}=a_{0} E_{\mathrm{x}}^{\mathrm{exact}}+\left(1-a_{0}\right) E_{\mathrm{x}}^{\mathrm{LSDA}}+a_{\mathrm{x}} \Delta E_{\mathrm{x}}^{\mathrm{B} 88}+\left(1-a_{\mathrm{c}}\right) E_{\mathrm{c}}^{\mathrm{VWN}-\mathrm{RPA}}+a_{\mathrm{c}} E_{\mathrm{c}}^{\mathrm{LYP}},
$$

where $a_{0}, a_{\mathrm{x}}$, and $a_{\mathrm{c}}$ have the same values as in B3PW91. Because the LYP correlation functional is not of the "LSDA + gradient correction" form, it cannot be naturally separated into local and nonlocal parts. This is why the coefficient $a_{\mathrm{c}}$ in Eq. (185) multiplies the entire LYP correlation energy, not the gradient correction for correlation, as in B3PW91. To avoid double-counting, the amount of local correlation is reduced by $a_{\mathrm{c}}$. It is this VWN term that became the source of confusion. When defining B3LYP, Stephens et al. [235] had in mind: (a) Eq. (73) with the parameters fitted to the exact Ceperley-Alder data; (b) the VWN interpolation scheme of Eq. (77). Instead, B3LYP was implemented: (a) with the parameters of Eq. (73) fitted to reproduce the random-phase approximation (RPA) values of the correlation energy of the uniform electron gas; (b) using the obsolete Barth-Hedin interpolation formula of Eq. (76). The Barth-Hedin interpolation is "form I" of the VWN paper, not "form III", as is stated in the GAUSSIAN manual and in the literature [236]. The correct VWN parametrization of the LSDA correlation energy was added to the GAUSSIAN program later along with the keyword VWN5 (the parametrization was referred to as "form V" in the VWN paper). The parameters of the intended VWN and the implemented VWNRPA forms are listed in Table 1. All other terms in Eq. (185) are unambiguous. Hertwig and Koch [236] compared both variants of B3LYP and found that, luckily, the "wrong" B3LYP is actually more accurate! 
The B3P86 hybrid also uses VWN-RPA as the local correlation component

$$
E_{\mathrm{xc}}^{\mathrm{B} 3 \mathrm{P} 86}=a_{0} E_{\mathrm{x}}^{\mathrm{exact}}+\left(1-a_{0}\right) E_{\mathrm{x}}^{\mathrm{LSDA}}+a_{\mathrm{x}} \Delta E_{\mathrm{x}}^{\mathrm{B} 88}+E_{\mathrm{c}}^{\mathrm{VWN}-\mathrm{RPA}}+a_{\mathrm{c}} \Delta E_{\mathrm{c}}^{\mathrm{P} 86},
$$

where $\Delta E_{\mathrm{c}}^{\mathrm{P} 86}$ is the second term on the right-hand side of Eq. (122), and the coefficients $a_{0}, a_{\mathrm{x}}$, and $a_{\mathrm{c}}$ have the same values as in B3PW91. The original B3PW91 functional was designed to be exact for a uniform density. In contrast, neither B3LYP nor B3P86 has this property, because LYP and VWN-RPA energies are not exact for a uniform electron gas.

In the wake of the success of B3PW91, Becke also introduced [168] a one-parameter simplification of Eq. (183),

$$
E_{\mathrm{xc}}=a_{0} E_{\mathrm{x}}^{\text {exact }}+\left(1-a_{0}\right) E_{\mathrm{x}}^{\mathrm{DFT}}+E_{\mathrm{c}}^{\mathrm{DFT}},
$$

which proved to be almost as accurate as three-parameter hybrids. Perdew, Ernzerhof and Burke [237] provided a theoretical justification for one-parameter GGA hybrids and gave a theoretical estimate of the mixing coefficient suitable for calculations of atomization energies of typical molecules: $a_{0}=1 / 4$. A rigorous formal treatment of hybrid schemes was also given by Görling and Levy [238].

Adamo and Barone [239] empirically readjusted two parameters of the PW91 exchange based on an analysis of the low-density, large-gradient, regions important in van der Waals systems, and combined their modified functional ( $m$ PW91) with the PW91 correlation to form a one-parameter hybrid, named $m \mathrm{PW} 1 \mathrm{PW} 91$. This functional is given by Eq. (187) with $E_{\mathrm{x}}^{\mathrm{DFT}}=E_{\mathrm{x}}^{m \mathrm{PW} 91}, E_{\mathrm{c}}^{\mathrm{DFT}}=E_{\mathrm{c}}^{\mathrm{PW} 91}$, and $a_{0}=0.25$.

A one-parameter hybrid version of the PBE exchange-correlation functional, called here PBEh (other names of the same functional include PBE0 and PBE1PBE) was introduced by Ernzerhof and Scuseria [240] and extensively studied by Adamo and Barone [241]. It has the form of Eq. (187) with DFT=PBE and $a_{0}=0.25$.

The hybrid TPSS functional (TPSSh) [180] is also given by Eq. (187) with DFT=TPSS and the mixing parameter $a_{0}=0.10$, which was determined by minimizing the mean absolute error in the enthalpies of formation of 223 G3/99 molecules. TPSSh satisfies the same exact constraints as the original TPSS. The fact that the value of $a_{0}$ in TPSSh is smaller than for a typical GGA hybrid suggests that meta-GGAs are better approximations to the exact exchange functional than GGAs.

Owing to ease of construction and agreeable performance, empirical hybrid functionals of the "mix-and-optimize" type have been proliferating in the literature over the past few years. These are functionals of the form

$$
E_{\mathrm{xc}}=E_{\mathrm{x}}+E_{\mathrm{c}}+a_{\mathrm{x}} E_{\mathrm{x}}^{\mathrm{exact}}
$$

where $E_{\mathrm{x}}$ and $E_{\mathrm{c}}$, are optimized simultaneously with $a_{\mathrm{x}}$, an empirical parameter. Examples of such hybrids include B97-1 [219], revised in Ref. [230], B97-2 [221], the Schmider-Becke hybrid GGA (SB98h) [222], $\tau$-SB98h [223], the $\tau$-HCTH hybrid [226], and the Boese-Martin functional for kinetics (BMK) [242].

Cohen and Handy [243] presented a hybrid functional similar to B3LYP that is based on the exchange functional OPTX of Eq. (180). This functional, named O3LYP, is given by

$$
E_{\mathrm{xc}}^{\mathrm{O} 3 \mathrm{LYP}}=a_{0} E_{\mathrm{x}}^{\text {exact }}+b_{0} E_{\mathrm{x}}^{\mathrm{LSDA}}+a_{\mathrm{x}} \Delta E_{\mathrm{x}}^{\mathrm{OPTX}}+\left(1-a_{\mathrm{c}}\right) E_{\mathrm{c}}^{\mathrm{VWN}}+a_{\mathrm{c}} E_{\mathrm{c}}^{\mathrm{LYP}},
$$


where $a_{0}=0.1161, b_{0}=0.9262, a_{\mathrm{x}}=0.8133$, and $a_{\mathrm{c}}=0.81$. Numerical studies show that O3LYP is overall more accurate than B3LYP for atoms and molecules, although not by much $[229,244-246]$. Note also that the fractions of approximate and exact exchange in Eqs. (188) and (189) no longer add up to 1. This means that the exchange components of these functionals do not reduce to $E_{\mathrm{x}}^{\mathrm{LSDA}}$ for uniform densities.

Very recently, Xu and Goddard [231] introduced a B3LYP-style hybrid version of their "extended" GGA functional of Eq. (181). Termed X3LYP, this functional uses reoptimized parameters in the $\Delta E_{\mathrm{x}}^{\mathrm{XG}}$ part $\left[a_{1}=0.675, a_{2}=0.235\right.$ in Eq. (181) $]$ and is given by

$$
E_{\mathrm{xc}}^{\mathrm{X} 3 \mathrm{LYP}}=a_{0} E_{\mathrm{x}}^{\mathrm{exact}}+\left(1-a_{0}\right) E_{\mathrm{x}}^{\mathrm{LSDA}}+a_{\mathrm{x}} \Delta E_{\mathrm{x}}^{\mathrm{XG}}+\left(1-a_{\mathrm{c}}\right) E_{\mathrm{c}}^{\mathrm{VWN}-\mathrm{RPA}}+a_{\mathrm{c}} E_{\mathrm{c}}^{\mathrm{LYP}},
$$

where $a_{0}=0.218, a_{\mathrm{x}}=0.709$, and $a_{\mathrm{c}}=0.871$ are also reoptimized. The performance of X3LYP appears to be comparable or better than that of other hybrid functionals [231].

A one-parameter hybrid functional combining the B88 exchange and B95 correlation has been proposed by Zhao et al. [247]. In another recent work, Zhao et al. [248] initiated the development of "doubly hybrid" functionals - empirical linear combinations of the HartreeFock, approximate DFT, and ab initio (e.g., MP2) energy terms.

\subsection{Local hybrids}

Although conventional hybrid functionals offer the best accuracy, they suffer from the same problems that plague GGAs and meta-GGAs, especially the self-interaction error (SIE). The SIE arises from inexact cancellation of spurious Coulomb self-interaction energy by approximate exchange. The exact exchange functional is, of course, self-interaction free. Inclusion of a fraction of exact exchange in global hybrid functionals alleviates, but does not solve completely, this problem. Adding a self-interaction correction [77] to the existing functionals to satisfy the constraint of Eq. (44) is an interesting method [249], but it is not straightforward to implement and apply.

A different approach to this problem is offered by local hybrid (Lh) [93] or hyper-GGA [72] scheme, in which the amount of approximate and exact exchange is determined by the local mixing function

$$
E_{\mathrm{xc}}^{\mathrm{Lh}}=\int\left\{a(\mathbf{r}) e_{\mathrm{x}}^{\mathrm{exact}}(\mathbf{r})+[1-a(\mathbf{r})] e_{\mathrm{x}}^{\mathrm{DFT}}(\mathbf{r})+e_{\mathrm{c}}^{\mathrm{DFT}}(\mathbf{r})\right\} d \mathbf{r},
$$

where $e_{\mathrm{x}}^{\text {exact }}(\mathbf{r})$ is the exact exchange energy density. The mixing function $a(\mathbf{r})$ must assume values between 0 and 1 and reduce to 1 for any one-electron density. This ensures that the self-interaction error is eliminated in one-electron-like regions which are particularly sensitive to the SIE. The local hybrid scheme can also be viewed as a generalization of global hybrid functionals.

The simplest function that fulfills these requirements is due to Becke [169]

$$
a(\mathbf{r})=\frac{\tau_{W}(\mathbf{r})}{\tau(\mathbf{r})} .
$$

Its use in the above local hybrid scheme was first reported by Jaramillo et al. [93]. This particular choice of $a(\mathbf{r})$ improves equilibrium geometries and binding energies of two-center, odd-electron symmetric cations such as $\mathrm{H}_{2}^{+}, \mathrm{He}_{2}^{+},(\mathrm{HF})_{2}^{+}$(the notorious problem discussed, for 
example, in Refs. [250] and [251]), and hydrogen abstraction barriers, but gives disappointing results for atomization energies [93]. The search for better mixing functions will certainly continue in the future [72]. It is clear that a local hybrid, if designed well, cannot be worse than the parent global hybrid.

\subsection{Screened hybrids}

Screened Coulomb interaction techniques find many interesting applications in modern quantum chemistry. For example, Gill and coworkers [252-256] describe an algorithm aimed at speeding up the computation of the Coulomb energy based on splitting the Coulomb operator $1 / u$ (where $u \equiv r_{12}$ ) into short-range (SR) and long-range (LR) components:

$$
\frac{1}{u}=\underbrace{\frac{1-\operatorname{erf}(\omega u)}{u}}_{\mathrm{SR}}+\underbrace{\frac{\operatorname{erf}(\omega u)}{u}}_{\mathrm{LR}},
$$

where $\operatorname{erf}(\omega u)$ is the error function and $\omega$ is an adjustable parameter. Their idea is to approximate the slowly-decaying $1 / u$ operator with the fast-decaying $\mathrm{SR}$ term plus corrections for the LR part. This reduces dramatically the required number of two-electron integrals. Savin and coworkers [257-260] use Coulomb screening to handle atomic and molecular neardegeneracy effects by combining multideterminantal wave functions with density functionals.

In the context of DFT, the partitioning of $1 / u$ is equivalent to representing $E_{\mathrm{xc}}$ as

$$
E_{\mathrm{xc}}=E_{\mathrm{xc}}^{\mathrm{SR}}(\omega)+E_{\mathrm{xc}}^{\mathrm{LR}}(\omega) .
$$

The short-range component of exact exchange is obtained from electron repulsion integrals calculated with the screened Coulomb potential:

$$
\langle\mu \nu \mid \lambda \sigma\rangle^{\mathrm{SR}}=\int d \mathbf{r}_{1} \int \chi_{\mu}^{*}\left(\mathbf{r}_{1}\right) \chi_{\nu}^{*}\left(\mathbf{r}_{2}\right) \frac{1-\operatorname{erf}\left(\omega r_{12}\right)}{r_{12}} \chi_{\lambda}\left(\mathbf{r}_{1}\right) \chi_{\sigma}\left(\mathbf{r}_{2}\right) d \mathbf{r}_{2} .
$$

To evaluate the long-range part of the DFT exchange energy, one inserts the screened Coulomb potential into Eq. (33) to obtain

$$
E_{\mathrm{x}}^{\mathrm{DFT}, \mathrm{LR}}(\omega)=2 \pi \int d \mathbf{r} \int_{0}^{\infty} \operatorname{erf}(\omega u) u h_{\mathrm{x}}(\mathbf{r}, u) d u .
$$

For example, for the LDA exchange this gives [258,261]

$$
E_{\mathrm{x}}^{\mathrm{LDA}, \mathrm{LR}}(\omega)=C_{\mathrm{x}} \int \rho^{4 / 3} \frac{2 \xi}{3}\left[2 \sqrt{\pi} \operatorname{erf}\left(\frac{1}{\xi}\right)-3 \xi+\xi^{3}+\left(2 \xi-\xi^{3}\right) e^{-1 / \xi^{2}}\right] d \mathbf{r},
$$

where $\xi=\omega / k_{F}$.

Recently, Heyd et al. [262] used the screening of $1 / u$ to adapt global hybrid functionals to calculations of periodic systems with small band gaps. Such an adaptation is necessary because direct evaluation of exact exchange in periodic systems with metallic character is prohibitively expensive, rendering conventional hybrids utterly impractical for many solids. Heyd et al. start with rewriting the one-parameter global hybrid functional of Eq. (187) as

$$
E_{\mathrm{xc}}^{\omega \mathrm{DFTh}}=a\left[E_{\mathrm{x}}^{\mathrm{exact}, \mathrm{SR}}(\omega)+E_{\mathrm{x}}^{\text {exact,LR }}(\omega)\right]+(1-a) E_{\mathrm{x}}^{\mathrm{DFT}}+E_{\mathrm{c}}^{\mathrm{DFT}},
$$


choose $\mathrm{DFT}=\mathrm{PBE}$, and then replace $E_{\mathrm{x}}^{\text {exact,LR }}(\omega)$, the slowest-decaying term, by $E_{\mathrm{x}}^{\mathrm{PBE}, \mathrm{LR}}(\omega)$ :

$$
E_{\mathrm{xc}}^{\omega \mathrm{PBEh}}=a\left[E_{\mathrm{x}}^{\mathrm{exact}, \mathrm{SR}}(\omega)+E_{\mathrm{x}}^{\mathrm{PBE}, \mathrm{LR}}(\omega)\right]+(1-a) E_{\mathrm{x}}^{\mathrm{PBE}}+E_{\mathrm{c}}^{\mathrm{PBE}} .
$$

Since the explicit PBE exchange hole is known [189], $E_{\mathrm{x}}^{\mathrm{PBE}, \mathrm{LR}}$ can be obtained analytically (for details, see Ref. [262]). For $a=1 / 4$ and $\omega=0$, the $\omega$ PBEh functional is equivalent to PBEh, while in the limit $\omega \rightarrow \infty$ it reduces to the PBE GGA. Extensive tests [262-264] show that $\omega \mathrm{PBEh}$ is an excellent approximation to the original $\mathrm{PBEh}$ functional and that the computational cost of $\omega \mathrm{PBEh}$ for solids is much closer to that of PBE than PBEh. A revised version of $\omega \mathrm{PBEh}$, called HSE [263], gives spectacular results in calculations of band gaps of semiconductors [264].

Other researchers [265-268] have used Coulomb screening to describe long-range interactions between atoms and molecules within hybrid DFT. In these methods, the LR component of exact exchange is kept, yielding the correct tails of the exchange potential and energy density, while the SR part is approximated by GGA or meta-GGA functionals. The fraction of exact exchange increases with $r_{12}$, which is precisely the opposite of the trend in Eq. (199). The resulting LR-corrected approximations, called "Coulomb-attenuated hybrids" [267], show improved performance for polarizabilities of long chains, Rydberg excitations, charge transfer, and van der Waals interactions.

Eq. (196) represents the proper way of screening exchange but, unfortunately, it requires knowing the explicit expression for the exchange hole. For most exchange functionals, $h_{\mathrm{x}}(\mathbf{r}, u)$ is not known. In such cases, the screened DFT exchange energy can be approximated as

$$
E_{\mathrm{x}}^{\mathrm{DFT}, \mathrm{LR}}(\omega) \approx \int e_{\mathrm{x}}^{\mathrm{LDA}, \mathrm{LR}}(\rho, \omega) F_{\mathrm{x}}^{\mathrm{DFT}}(\rho, \nabla \rho, \ldots) d \mathbf{r}
$$

where $e_{\mathrm{x}}^{\mathrm{LDA}, \mathrm{LR}}$ is the exchange energy density in Eq. (197) and $F_{\mathrm{x}}$ is a GGA or meta-GGA enhancement factor. Although Coulomb screening of a density functional by Eq. (200) is only an approximation to the exact procedure, it is easy to implement for almost any exchange functional and has been commonly used in practice [265-268].

\section{Implementation and performance}

The self-consistent field procedure in Kohn-Sham DFT is very similar to that of the conventional Hartree-Fock method [269]. The main difference is that the functional $E_{\mathrm{xc}}[\rho]$ and matrix elements of $v_{\mathrm{xc}}(\mathbf{r})$ have to be evaluated in Kohn-Sham DFT numerically, whereas the Hartree-Fock method is entirely analytic. Efficient formulas for computing matrix elements of $v_{\mathrm{xc}}(\mathbf{r})$ in finite basis sets have been developed [270,271], along with accurate numerical integration grids [272-277] and techniques for real-space grid integration [278, 279].

For an exchange-correlation functional of the form

$$
E_{\mathrm{xc}}[\rho]=\int e_{\mathrm{xc}}\left(\rho,|\nabla \rho|, \nabla^{2} \rho\right) d \mathbf{r}
$$

the exchange-correlation potential is given by the rules of calculus of variations [280]

$$
v_{\mathrm{xc}} \equiv \frac{\delta E_{\mathrm{xc}}}{\delta \rho}=\frac{\partial e_{\mathrm{xc}}}{\partial \rho}-\nabla \cdot\left(\frac{\partial e_{\mathrm{xc}}}{\partial \nabla \rho}\right)+\nabla^{2}\left(\frac{\partial e_{\mathrm{xc}}}{\partial \nabla^{2} \rho}\right) \text {. }
$$


If $e_{\mathrm{xc}}$ also depends on $\phi_{i}$ or $\tau$, then explicit differentiation with respect to $\rho$ is no longer possible and the optimized effective potential (OEP) method [47-55] should be used. In practice, the determination of the OEP is often avoided by departing slightly from the true Kohn-Sham scheme and minimizing the energy functional with respect to Kohn-Sham orbitals [271], that is, by assuming

$$
v_{\mathrm{xc}} \phi_{i}=\frac{\delta E_{\mathrm{xc}}}{\delta \phi_{i}^{*}}
$$

For functionals having the form of Eq. (201), both approaches are equivalent because by the chain rule

$$
\frac{\delta E_{\mathrm{xc}}}{\delta \phi_{i}^{*}}=\int \frac{\delta E_{\mathrm{xc}}}{\delta \rho\left(\mathbf{r}^{\prime}\right)} \frac{\delta \rho\left(\mathbf{r}^{\prime}\right)}{\delta \phi_{i}^{*}(\mathbf{r})} d \mathbf{r}^{\prime}=\int \frac{\delta E_{\mathrm{xc}}}{\delta \rho\left(\mathbf{r}^{\prime}\right)} \frac{\partial \rho}{\partial \phi_{i}^{*}} \delta\left(\mathbf{r}-\mathbf{r}^{\prime}\right) d \mathbf{r}^{\prime}=\frac{\delta E_{\mathrm{xc}}}{\delta \rho} \frac{\partial \rho}{\partial \phi_{i}^{*}}=\frac{\delta E_{\mathrm{xc}}}{\delta \rho} \phi_{i} .
$$

In the general case of orbital-dependent functionals, minimization with respect to orbitals is only an approximation to the true Kohn-Sham scheme [281-285] (see also Ref. [58] concerning the gauge invariance problem with conventional $\tau$-dependent functionals).

Table 2 compares the performance of most density functionals discussed in this review for selected atomic and molecular properties. The list of molecules comprising the G3 thermochemical test set is given in Refs. [286] and [287]. The G3/IP, G3/EA, and G3/PA test sets are described in Ref. [288]. The T-96R and T-82F sets are defined in Ref. [180]. All other computational details can be found in Ref. [180]. The results in Table 2 show that LSDA is already a great improvement over the Hartree-Fock method. GGA functionals further increase the accuracy for molecular binding energies, electron and proton affinities. Meta-GGAs and hybrid functionals generally provide the most accurate predictions for all properties. Note that the recent nonempirical functionals are now vying with the best fitted functionals for the top places in the performance ranking. Systematic numerical studies of density functionals for other properties regularly appear in the literature [181, 194, 246, 288-293].

\section{Conclusion}

Back in the 1960s, hopes for future progress in electronic structure theory were associated with correlated wave function techniques and the tantalizing possibility of variational calculations based on the two-electron reduced density matrix [294]. Density functional theory was not on the quantum chemistry agenda at that time. The progress of wave function techniques has been remarkable, as documented elsewhere in this volume. In contrast, the density matrix approach has not yet materialized into a competitive computational method, despite many persistent efforts [295]. Meanwhile, approximate DFT has become the most widely used method of quantum chemistry, offering an unprecedented accuracy-to-cost ratio.

A few personal reminiscences of the time when DFT was entering mainstream quantum chemistry are appropriate here. In March of 1992, while the first author of this review was actively working on coupled cluster theory, John Pople visited Rice University to deliver the Franklin Memorial Lecture. The subject of his talk was the impressive performance of the BLYP functional for thermochemistry, especially as judged by its low computational cost relative to the G2 theory. Pople was on his way to the Sanibel Symposium and gave the author a preprint of Ref. [296], which later became Pople's first publication on DFT (see 
Table 2: Mean absolute deviations (m.a.d.) from experiment for standard enthalpies of formation $\left(\Delta_{\mathrm{f}} H_{298}^{\circ}\right)$, ionization potentials (IP), electron affinities (EA), proton affinities (PA), equilibrium bond lengths $\left(r_{e}\right)$, and harmonic vibrational frequencies $\left(\omega_{e}\right)$ computed with approximate functionals using the 6-311++G(3df,3pd) basis set. The fully nonempirical functionals in this table are HF, LSDA, PW91, PBE, and TPSS.

\begin{tabular}{|c|c|c|c|c|c|c|}
\hline \multirow[b]{3}{*}{ Method } & \multicolumn{6}{|c|}{ Property (units of m.a.d.) / Test set (size) } \\
\hline & $\Delta_{\mathrm{f}} H_{298}^{\circ}(\mathrm{kcal} / \mathrm{mol})$ & IP $(e V)$ & $\mathrm{EA}(\mathrm{eV})$ & $\mathrm{PA}(\mathrm{kcal} / \mathrm{mol})$ & $r_{e}(\AA)$ & $\omega_{e}\left(\mathrm{~cm}^{-1}\right)$ \\
\hline & G3 (223) & G3/IP (86) & G3/EA (58) & G3/PA (8) & T-96R (96) & $\mathrm{T}-82 \mathrm{~F}(82)$ \\
\hline $\mathrm{HF}$ & 211.5 & 1.03 & 1.10 & 3.1 & $0.025^{a}$ & $136.2^{a}$ \\
\hline $\operatorname{LSDA}^{b}$ & 121.4 & 0.23 & 0.24 & 5.9 & $0.013^{c}$ & $48.9^{d}$ \\
\hline \multicolumn{7}{|c|}{ Generalized gradient approximations } \\
\hline BLYP & 9.5 & 0.29 & 0.12 & 1.6 & 0.022 & 55.2 \\
\hline BPW91 & 9.0 & 0.24 & 0.11 & 1.4 & 0.017 & 41.4 \\
\hline BP86 & 26.3 & 0.21 & 0.19 & 1.3 & 0.017 & 45.5 \\
\hline PW91 & 23.6 & 0.22 & 0.14 & 1.6 & 0.014 & 39.8 \\
\hline PBE & 22.2 & 0.24 & 0.12 & 1.6 & 0.016 & 42.0 \\
\hline НCTH/407 & 7.2 & 0.23 & 0.19 & 1.9 & 0.014 & 39.9 \\
\hline OLYP & 5.9 & 0.29 & 0.15 & 1.7 & 0.018 & 40.2 \\
\hline \multicolumn{7}{|c|}{ Meta-generalized gradient approximations } \\
\hline VS98 & 3.5 & 0.23 & 0.13 & 1.6 & 0.013 & 33.9 \\
\hline BKCIS & 7.2 & 0.22 & 0.20 & 1.5 & $0.019^{e}$ & $45.1^{e}$ \\
\hline PKZB & 7.0 & 0.31 & 0.15 & 1.8 & 0.027 & 51.7 \\
\hline TPSS & 5.8 & 0.24 & 0.14 & 1.8 & 0.014 & 30.4 \\
\hline $\begin{array}{l}\text { BRxB } 88 \mathrm{c}^{f} \\
\text { Hybrid fund }\end{array}$ & ionals & 0.22 & 0.14 & 2.7 & 0.026 & 48.7 \\
\hline B3PW91 & 3.9 & 0.19 & 0.14 & 1.1 & 0.009 & 36.2 \\
\hline B3LYP & 4.9 & 0.18 & 0.12 & 1.2 & 0.010 & 33.5 \\
\hline B3P86 & 26.1 & 0.55 & 0.59 & 1.0 & 0.008 & 37.0 \\
\hline$m$ PW1PW91 & 4.1 & 0.19 & 0.16 & 1.1 & 0.010 & 42.9 \\
\hline PBEh & 6.7 & 0.20 & 0.17 & 1.1 & 0.010 & 43.6 \\
\hline HSE & 6.0 & 0.22 & 0.12 & 1.0 & 0.009 & 43.9 \\
\hline O3LYP & 3.7 & 0.22 & 0.13 & 1.5 & 0.012 & 33.7 \\
\hline B97-1 & 4.9 & 0.19 & 0.11 & 1.0 & 0.012 & 32.5 \\
\hline B97-2 & 4.7 & 0.18 & 0.14 & 2.2 & 0.012 & 40.9 \\
\hline $\mathrm{SB} 8 \mathrm{~h}^{g}$ & 3.9 & 0.18 & 0.11 & 0.9 & 0.012 & 33.8 \\
\hline TPSSh & 3.9 & 0.23 & 0.16 & 1.8 & 0.010 & 26.7 \\
\hline
\end{tabular}

${ }^{a}$ Excludes $\mathrm{Be}_{2}$ (no binding).

${ }^{b}$ Using the Perdew-Wang representation of the LSDA correlation energy [114].

${ }^{c}$ Excludes $\mathrm{F}_{2}^{+}$and $\mathrm{SF}$ (no convergence).

${ }^{d}$ Excludes $\mathrm{F}_{2}^{+}$(no convergence).

${ }^{e}$ Excludes $\mathrm{C}_{2}$ (no convergence).

$f$ From Ref. [188].

$g$ Fit 2c in Table III of Ref. [222]. The Gaussian keyword is B98.

also Ref. [297]). Pople credited the lecture by Axel Becke at the 7th International Congress of Quantum Chemistry in Menton in the Summer of 1991 as a turning point in his views on DFT. With the help of Peter Gill by email correspondence, the author promptly added a numerical quadrature code to his Hartree-Fock program and was comparing coupled-cluster results to DFT in a matter of weeks [298].

Impressive as the performance of modern density functionals is, none of the practical approximations is still suitable for general use. LSDA, GGAs, meta-GGAs, and global hybrids all systematically underperform or simply fail for several broad classes of problems 
such as: left-right correlation, van der Waals interactions, negative ions, orbital spectra and band structure, open-shell singlet diradicals, etc. Even for thermochemistry of small molecules, none of the currently available density functionals has yet achieved an accuracy better than a few $\mathrm{kcal} / \mathrm{mol}$. In principle, there is no doubt that DFT can describe all such systems, provided that accurate Kohn-Sham exchange-correlation potentials and functionals are used. This has been demonstrated convincingly by generating the exact $v_{\text {xc }}(\mathbf{r})$ via $a b$ initio methods and solving the corresponding Kohn-Sham equations [52, 91, 299-304]. Given the formidable complexity of the true exchange-correlation functional and the magnitude of the reward, development of better approximations is certain to remain a "promising and charming" subject for years to come.

Acknowledgments. We are very grateful to John Perdew, Jianmin Tao, Andreas Savin, Sergey Maximoff, Oleg Vydrov, and Artur Izmaylov for comments and suggestions about our manuscript. This work was supported by the National Science Foundation, the Welch Foundation, and the Department of Energy.

\section{References}

[1] R. G. Parr, Ann. Rev. Phys. Chem. 34 (1983) 631.

[2] E. Fermi, Z. Phys. 48 (1928) 73; reprinted in English in Ref. [6].

[3] P. A. M. Dirac, Proc. Camb. Phil. Soc. 26 (1930) 376.

[4] P. Gombás, Die Statistische Theorie des Atoms und Ihre Anwendungen, Springer-Verlag, Wien, 1949.

[5] N. H. March, Adv. Phys. 6 (1957) 1.

[6] N. H. March, Self-consistent Fields in Atoms: Hartree and Thomas-Fermi Atoms, Pergamon, Oxford, 1975.

[7] E. H. Lieb, Rev. Mod. Phys. 53 (1981) 603; ibid. 54 (1982) 311(E).

[8] R. Gáspár, Acta Phys. Hung. 3 (1954) 263.

[9] J. C. Slater, Phys. Rev. 81 (1951) 385.

[10] J. C. Slater, Adv. Quantum Chem. 6 (1972) 1.

[11] J. C. Slater, Int. J. Quantum Chem. Symp. 9 (1975) 7.

[12] E. Teller, Rev. Mod. Phys. 34 (1962) 627.

[13] N. L. Balàzs, Phys. Rev. 156 (1967) 42.

[14] I. Catto, P. L. Lions, Commun. Part. Diff. Eq. 17 (1992) 1051.

[15] P. Hohenberg, W. Kohn, Phys. Rev. 136 (1964) B864.

[16] W. Kohn, in: F. Bassani, F. Fumi, M. P. Tosi (Eds.), Highlights of Condensed-Matter Theory, North-Holland, Amsterdam, 1985.

[17] W. Kohn, L. J. Sham, Phys. Rev. 140 (1965) A1133.

[18] W. Kohn, A. D. Becke, R. G. Parr, J. Phys. Chem. 100 (1996) 12974.

[19] R. G. Parr, W. Yang, Ann. Rev. Phys. Chem. 46 (1995) 701.

[20] R. G. Parr, W. Yang, Density-Functional Theory of Atoms and Molecules, Oxford University Press, New York, 1989.

[21] P. M. W. Gill, in: P. von R. Schleyer (Ed.), Encyclopedia of Computational Chemistry, John Wiley \& Sons, New York, 1998, p. 678.

[22] W. Koch, M. C. Holthausen, A Chemist's Guide to Density Functional Theory, Wiley-VCH, Weinheim, 2000. 
[23] J. P. Perdew, S. Kurth, in: C. Fiolhais, F. Nogueira, M. Marques (Eds.), A Primer in Density Functional Theory, Springer, Berlin, 2003.

[24] J. Kohanoff, N. I. Gidopoulos, in: S. Wilson (Ed.), Handbook of Molecular Physics and Quantum Chemistry, Vol. 2: Molecular Electronic Structure, John Wiley \& Sons, Chichester, 2003.

[25] J. P. Dahl, J. Avery (Eds.), Local Density Approximations in Quantum Chemistry and Solid State Physics, Plenum, New York, 1984.

[26] R. M. Dreizler, J. da Providência (Eds.), Density Functional Methods in Physics (NATO ASI Series, Vol. B123), Plenum, New York, 1985.

[27] R. M. Dreizler, E. K. U. Gross, Density Functional Theory, Springer, Berlin, 1990.

[28] E. S. Kryachko, E. V. Ludeña, Energy Density Functional Theory of Many-Electron Systems, Kluwer, Dordrecht, 1990.

[29] E. K. U. Gross, R. M. Dreizler (Eds.), Density Functional Theory (NATO ASI Series, Vol. B337), Plenum, New York, 1995.

[30] J. M. Seminario, P. Politzer (Eds.), Modern Density Functional Theory: A Tool for Chemistry, Elsevier, Amsterdam, 1995.

[31] J. M. Seminario (Ed.), Recent Developments and Applications of Modern Density Functional Theory, Elsevier, Amsterdam, 1996.

[32] Gaussian 03, Revision C.2, M. J. Frisch, G. W. Trucks, H. B. Schlegel, G. E. Scuseria, M. A. Robb, J. R. Cheeseman, J. A. Montgomery, Jr., T. Vreven, K. N. Kudin, J. C. Burant, J. M. Millam, S. S. Iyengar, J. Tomasi, V. Barone, B. Mennucci, M. Cossi, G. Scalmani, N. Rega, G. A. Petersson, H. Nakatsuji, M. Hada, M. Ehara, K. Toyota, R. Fukuda, J. Hasegawa, M. Ishida, T. Nakajima, Y. Honda, O. Kitao, H. Nakai, M. Klene, X. Li, J. E. Knox, H. P. Hratchian, J. B. Cross, C. Adamo, J. Jaramillo, R. Gomperts, R. E. Stratmann, O. Yazyev, A. J. Austin, R. Cammi, C. Pomelli, J. W. Ochterski, P. Y. Ayala, K. Morokuma, G. A. Voth, P. Salvador, J. J. Dannenberg, V. G. Zakrzewski, S. Dapprich, A. D. Daniels, M. C. Strain, O. Farkas, D. K. Malick, A. D. Rabuck, K. Raghavachari, J. B. Foresman, J. V. Ortiz, Q. Cui, A. G. Baboul, S. Clifford, J. Cioslowski, B. B. Stefanov, G. Liu, A. Liashenko, P. Piskorz, I. Komaromi, R. L. Martin, D. J. Fox, T. Keith, M. A. Al-Laham, C. Y. Peng, A. Nanayakkara, M. Challacombe, P. M. W. Gill, B. Johnson, W. Chen, M. W. Wong, C. Gonzalez, and J. A. Pople, Gaussian, Inc., Wallingford, CT, 2004.

[33] Y. A. Wang, E. A. Carter, in: S. D. Schwartz (Ed.), Theoretical Methods in Condensed Phase Chemistry, Kluwer Academic, Dordrecht, 2000.

[34] V. Sahni, Quantal Density Functional Theory, Springer, Berlin, 2004.

[35] E. V. Ludeña, E. S. Kryachko, T. Koga, R. López-Boada, J. Hinze, J. Maldonado, E. Valderrama, in: J. M. Seminario, P. Politzer (Eds.), Modern Density Functional Theory: A Tool for Chemistry (Theoretical and Computational Chemistry, Vol. 2), Elsevier, Amsterdam, 1995, p. 75 .

[36] M. Levy, Proc. Natl. Acad. Sci. USA 76 (1979) 6062.

[37] S. S. Iyengar, M. Ernzerhof, S. N. Maximoff, G. E. Scuseria, Phys. Rev. A 63 (2001) 052508.

[38] P. C. Hohenberg, W. Kohn, L. J. Sham, Adv. Quantum Chem. 21 (1990) 7.

[39] W. Kohn, Rev. Mod. Phys. 71 (1999) 1253.

[40] A. Savin, F. Colonna, R. Pollet, Int. J. Quantum Chem. 93 (2003) 166.

[41] S. T. Epstein, in: B. M. Deb (Ed.), The Force Concept in Chemistry, Van Nostrand, New York, 1981.

[42] J. Harris, R. O. Jones, J. Phys. F 4 (1974) 1170.

[43] D. C. Langreth, J. P. Perdew, Solid State Commun. 17 (1975) 1425.

[44] D. C. Langreth, J. P. Perdew, Phys. Rev. B 15 (1977) 2884. 
[45] O. Gunnarsson, B. I. Lundqvist, Phys. Rev. B 13 (1976) 4274.

[46] R. McWeeny, B. T. Sutcliffe, Methods of Molecular Quantum Mechanics, Academic Press, London, 1969.

[47] R. T. Sharp, G. K. Horton, Phys. Rev. 90 (1953) 317.

[48] J. D. Talman, W. F. Shadwick, Phys. Rev. A 14 (1976) 36.

[49] T. Grabo, T. Kreibich, S. Kurth, E. K. U. Gross, in: V. I. Anisimov (Ed.), Strong Coulomb Correlations in Electronic Structure Calculations, Gordon and Breach, Amsterdam, 2000.

[50] S. Ivanov, S. Hirata, R. J. Bartlett, Phys. Rev. Lett. 83 (1999) 5455.

[51] S. Ivanov, S. Hirata, R. J. Bartlett, J. Chem. Phys. 116 (2002) 1269.

[52] E. Engel, R. M. Dreizler, J. Comput. Chem. 20 (1999) 31.

[53] O. V. Gritsenko, E. J. Baerends, Phys. Rev. A 64 (2001) 042506.

[54] W. Yang, Q. Wu, Phys. Rev. Lett. 89 (2002) 143002.

[55] S. Kümmel, J. P. Perdew, Phys. Rev. B 68 (2003) 035103.

[56] U. von Barth, L. Hedin, J. Phys. C: Solid State Phys. 5 (1972) 1629.

[57] M. M. Pant, A. K. Rajagopal, Solid State Commun. 10 (1972) 1157.

[58] S. N. Maximoff, G. E. Scuseria, Chem. Phys. Lett. 390 (2004) 408.

[59] H. L. Schmider, A. D. Becke, J. Mol. Struct. (Theochem) 527 (2000) 51.

[60] H. L. Schmider, A. D. Becke, J. Chem. Phys. 116 (2002) 3184.

[61] S. N. Maximoff, M. Ernzerhof, G. E. Scuseria, J. Chem. Phys. 117 (2002) 3074.

[62] S. M. Colwell, N. C. Handy, Chem. Phys. Lett. 217 (1994) 271.

[63] A. M. Lee, S. M. Colwell, N. C. Handy, Chem. Phys. Lett. 229 (1994) 225.

[64] A. M. Lee, N. C. Handy, S. M. Colwell, J. Chem. Phys. 103 (1995) 10095.

[65] A. D. Becke, Can. J. Chem. 74 (1996) 995.

[66] A. D. Becke, J. Chem. Phys. 117 (2002) 6935.

[67] S. N. Maximoff, M. Ernzerhof, G. E. Scuseria, J. Chem. Phys. 120 (2004) 2105.

[68] G. Vignale, M. Rasolt, Phys. Rev. Lett. 59 (1987) 2360; ibid. 62 (1989) 115(E).

[69] G. Vignale, M. Rasolt, D. J. W. Geldart, Phys. Rev. B 37 (1988) 2502.

[70] G. Vignale, M. Rasolt, Phys. Rev. B 37 (1988) 10685.

[71] G. Vignale, M. Rasolt, D. J. W. Geldart, Adv. Quantum Chem. 21 (1990) 235.

[72] J. P. Perdew, K. Schmidt, in: V. Van Doren, C. Van Alsenoy, P. Geerlings (Eds.), Density Functional Theory and Its Application to Materials, AIP, Melville, NY, 2001.

[73] J. P. Perdew, A. Ruzsinszky, J. Tao, V. N. Staroverov, G. E. Scuseria, G. I. Csonka, J. Chem. Phys. 123 (2005) 062201.

[74] J. P. Perdew, M. Levy, Int. J. Quantum Chem. Symp. 49 (1994) 539.

[75] E. H. Lieb, S. Oxford, Int. J. Quantum Chem. 19 (1981) 427.

[76] J. P. Perdew, in: P. Ziesche, H. Eschrig (Eds.), Electronic Structure of Solids '91, Akademie, Berlin, 1991.

[77] J. P. Perdew, A. Zunger, Phys. Rev. B 23 (1981) 5048.

[78] M. Levy, J. P. Perdew, Phys. Rev. A 32 (1985) 2010.

[79] M. Levy, Int. J. Quantum Chem. Symp. 23 (1989) 617.

[80] H. Ou-Yang, M. Levy, Phys. Rev. A 42 (1990) 155.

[81] M. Levy, H. Ou-Yang, Phys. Rev. A 42 (1990) 651.

[82] M. Levy, Phys. Rev. A 43 (1991) 4637.

[83] A. Görling, M. Levy, Phys. Rev. A 45 (1992) 1509.

[84] M. Levy, J. P. Perdew, Phys. Rev. B 48 (1993) 11638.

[85] M. Levy, in: E. K. U. Gross, R. M. Dreizler (Eds.), Density Functional Theory, Plenum, New York, 1995. 
[86] G. L. Oliver, J. P. Perdew, Phys. Rev. A 20 (1979) 397.

[87] A. Görling, M. Levy, Phys. Rev. A 47 (1993) 13105.

[88] A. Görling, M. Levy, Phys. Rev. A 50 (1994) 196.

[89] A. Görling, M. Levy, Int. J. Quantum Chem. Symp. 29 (1995) 93.

[90] S. Ivanov, R. J. Bartlett, J. Chem. Phys. 114 (2001) 1952.

[91] I. Grabowski, S. Hirata, S. Ivanov, R. J. Bartlett, J. Chem. Phys. 116 (2002) 4415.

[92] S. Ivanov, S. Hirata, I. Grabowski, R. J. Bartlett, J. Chem. Phys. 118 (2003) 461.

[93] J. Jaramillo, G. E. Scuseria, M. Ernzerhof, J. Chem. Phys. 118 (2003) 1068.

[94] J. A. Alonso, L. A. Girifalco, Phys. Rev. B 17 (1978) 3735.

[95] O. Gunnarsson, M. Jonson, B. I. Lundqvist, Phys. Rev. B 20 (1979) 3136.

[96] M. D. Glossman, A. Rubio, L. C. Balbás, J. A. Alonso, Int. J. Quantum Chem. Symp. 26 (1992) 347.

[97] O. V. Gritsenko, N. A. Cordero, A. Rubio, L. C. Balbás, J. A. Alonso, Phys. Rev. A 48 (1993) 3197.

[98] S. K. Ghosh, M. Berkowitz, R. G. Parr, Proc. Natl. Acad. Sci. USA 81 (1984) 8028.

[99] S. K. Ghosh, R. G. Parr, Phys. Rev. A 34 (1986) 785.

[100] S. Liu, R. G. Parr, Phys. Rev. A 55 (1996) 1972.

[101] Y. A. Wang, S. Liu, R. G. Parr, Chem. Phys. Lett. 267 (1997) 14.

[102] A. Nagy, S. Liu, R. G. Parr, Phys. Rev. A 59 (1999) 3349.

[103] F. Bloch, Z. Phys. 57 (1929) 545.

[104] A. Sommerfeld, H. Bethe, in: H. Geiger, K. Scheel (Eds.), Handbuch der Physik, 2nd Ed., Springer, Berlin, 1933, p. 485.

[105] J. C. Slater, Quantum Theory of Atomic Structure, Vol. 2, McGraw-Hill, New York, 1960.

[106] E. Wigner, F. Seitz, Phys. Rev. 46 (1934) 509.

[107] M. Ernzerhof, G. E. Scuseria, J. Chem. Phys. 111 (1999) 911.

[108] M. Gell-Mann, K. A. Brueckner, Phys. Rev. 106 (1957) 364.

[109] W. J. Carr, Jr., A. A. Maradudin, Phys. Rev. 133 (1964) A371.

[110] P. Nozières, D. Pines, Phys. Rev. 111 (1958) 442.

[111] W. J. Carr, Jr., Phys. Rev. 122 (1961) 1437.

[112] D. M. Ceperley, B. J. Alder, Phys. Rev. Lett. 45 (1980) 566.

[113] S. H. Vosko, L. Wilk, M. Nusair, Can. J. Phys. 58 (1980) 1200.

[114] J. P. Perdew, Y. Wang, Phys. Rev. B 45 (1992) 13244.

[115] P. R. Antoniewicz, L. Kleinman, Phys. Rev. B 31 (1985) 6779.

[116] L. J. Sham, in: P. M. Marcus, J. F. Janak, A. R. Williams (Eds.), Computational Methods in Band Theory, Plenum Press, New York, 1971, p. 458.

[117] L. Kleinman, S. Lee, Phys. Rev. B 37 (1988) 4634.

[118] J. A. Chevary, S. H. Vosko, Phys. Rev. B 42 (1990) 5320.

[119] E. Engel, S. H. Vosko, Phys. Rev. B 50 (1994) 10498.

[120] P. S. Svendsen, U. von Barth, Phys. Rev. B 54 (1996) 17402.

[121] S.-K. Ma, K. A. Brueckner, Phys. Rev. 165 (1968) 18.

[122] M. Rasolt, D. J. W. Geldart, Phys. Rev. B 34 (1986) 1325.

[123] M. Ernzerhof, G. E. Scuseria, J. Chem. Phys. 112 (2000) 5270.

[124] F. Herman, J. P. Van Dyke, I. B. Ortenburger, Phys. Rev. Lett. 22 (1969) 807.

[125] A. D. Becke, J. Chem. Phys. 84 (1986) 4524.

[126] A. D. Becke, J. Chem. Phys. 85 (1986) 7184.

[127] A. E. DePristo, J. D. Kress, J. Chem. Phys. 86 (1987) 1425.

[128] A. D. Becke, Phys. Rev. A 38 (1988) 3098. 
[129] R. van Leeuwen, E. J. Baerends, Phys. Rev. A 49 (1994) 2421.

[130] P. Jemmer, P. J. Knowles, Phys. Rev. A 51 (1995) 3571.

[131] D. J. Tozer, N. C. Handy, J. Chem. Phys. 109 (1998) 10180.

[132] D. J. Tozer, N. C. Handy, Mol. Phys. 101 (2003) 2669.

[133] D. C. Langreth, J. P. Perdew, Phys. Rev. B 21 (1980) 5469.

[134] D. C. Langreth, M. J. Mehl, Phys. Rev. B 28 (1983) 1809.

[135] J. P. Perdew, Phys. Rev. Lett. 55 (1985) 1665; ibid. 55 (1985) 2370(E).

[136] J. P. Perdew, Y. Wang, Phys. Rev. B 33 (1986) 8800.

[137] N. H. March, Phys. Rev. A 36 (1987) 5077.

[138] F. Della Sala, A. Görling, Phys. Rev. Lett. 89 (2002) 033003.

[139] M. Abramowitz, I. A. Stegun, Handbook of Mathematical Functions with Formulas, Graphs, and Mathematical Tables, U.S. Government, Washington, D.C., 1972.

[140] D. J. Lacks, R. G. Gordon, Phys. Rev. A 47 (1993) 4681.

[141] C. Adamo, V. Barone, J. Comput. Chem. 19 (1998) 418.

[142] M. Filatov, W. Thiel, Mol. Phys. 91 (1997) 847.

[143] O. V. Gritsenko, R. van Leeuwen, E. van Lenthe, E. J. Baerends, Phys. Rev. A 51 (1995) 1944.

[144] O. V. Gritsenko, R. van Leeuwen, E. J. Baerends, Int. J. Quantum Chem. 61 (1997) 231.

[145] O. V. Gritsenko, P. R. T. Schipper, E. J. Baerends, Chem. Phys. Lett. 302 (1999) 199.

[146] P. R. T. Schipper, O. V. Gritsenko, S. J. A. van Gisbergen, E. J. Baerends, J. Chem. Phys. $112(2000) 1344$.

[147] O. V. Gritsenko, P. R. T. Schipper, E. J. Baerends, Int. J. Quantum Chem. 76 (2000) 407.

[148] E. Engel, J. A. Chevary, L. D. Macdonald, S. H. Vosko, Z. Phys. D 23 (1992) 7.

[149] M. Filatov, W. Thiel, Phys. Rev. A 57 (1998) 189.

[150] R. Neumann, N. C. Handy, Chem. Phys. Lett. 266 (1997) 16.

[151] P. M. W. Gill, Mol. Phys. 89 (1996) 433.

[152] M. Ernzerhof, S. N. Maximoff, G. E. Scuseria, J. Chem. Phys. 116 (2002) 3980.

[153] J. P. Perdew, Physica B 172 (1991) 1.

[154] K. Burke, J. P. Perdew, Int. J. Quantum Chem. 56 (1995) 199.

[155] K. Burke, J. P. Perdew, Y. Wang, in: J. F. Dobson, G. Vignale, M. P. Das (Eds.), Electronic Density Functional Theory: Recent Progress and New Directions, Plenum Press, New York, 1998.

[156] J. P. Perdew, K. Burke, Y. Wang, Phys. Rev. B 54 (1996) 16533; ibid. 57 (1998) 14999(E).

[157] J. P. Perdew, J. A. Chevary, S. H. Vosko, K. A. Jackson, M. R. Pederson, D. J. Singh, C. Fiolhais, Phys. Rev. B 46 (1992) 6671; ibid. 48 (1993) 4978(E).

[158] Y. Wang, J. P. Perdew, Phys. Rev. B 43 (1991) 8911.

[159] J. P. Perdew, K. Burke, M. Ernzerhof, Phys. Rev. Lett. 77 (1996) 3865; ibid. 78 (1997) 1396(E).

[160] C. D. Hu, D. C. Langreth, Phys. Scr. 32 (1985) 391.

[161] C. D. Hu, D. C. Langreth, Phys. Rev. B 33 (1986) 943.

[162] J. P. Perdew, Phys. Rev. B 33 (1986) 8822; ibid. 34 (1986) 7406(E).

[163] L. C. Wilson, M. Levy, Phys. Rev. B 41 (1990) 12930.

[164] V. N. Staroverov, G. E. Scuseria, J. P. Perdew, J. Tao, E. R. Davidson, Phys. Rev. A 70 (2004) 012502.

[165] Y. Zhang, W. Yang, Phys. Rev. Lett. 80 (1998) 890.

[166] J. P. Perdew, K. Burke, M. Ernzerhof, Phys. Rev. Lett. 80 (1998) 891.

[167] B. Hammer, L. B. Hansen, J. K. Nørskov, Phys. Rev. B 59 (1999) 7413. 
[168] A. D. Becke, J. Chem. Phys. 104 (1996) 1040.

[169] A. D. Becke, J. Chem. Phys. 109 (1998) 2092.

[170] H. Stoll, C. M. E. Pavlidou, H. Preuß, Theor. Chim. Acta 49 (1978) 143.

[171] H. Stoll, E. Golka, H. Preuß, Theor. Chim. Acta 55 (1980) 29.

[172] J. B. Krieger, J. Chen, G. J. Iafrate, A. Savin, in: A. Gonis, N. Kioussis, M. Ciftan (Eds.), Electron Correlations and Materials Properties, Kluwer/Plenum, New York, 1999.

[173] J. P. Perdew, R. G. Parr, M. Levy, J. L. Balduz, Jr., Phys. Rev. Lett. 49 (1982) 1691.

[174] J. Rey, A. Savin, Int. J. Quantum Chem. 69 (1998) 581.

[175] J. Toulouse, A. Savin, C. Adamo, J. Chem. Phys. 117 (2002) 10465.

[176] J. P. Perdew, S. Kurth, A. Zupan, P. Blaha, Phys. Rev. Lett. 82 (1999) 2544.

[177] C. Adamo, M. Ernzerhof, G. E. Scuseria, J. Chem. Phys. 112 (2000) 2643.

[178] A. Rabuck, G. E. Scuseria, Theor. Chem. Acc. 104 (2000) 439.

[179] J. Tao, J. P. Perdew, V. N. Staroverov, G. E. Scuseria, Phys. Rev. Lett. 91 (2003) 146401.

[180] V. N. Staroverov, G. E. Scuseria, J. Tao, J. P. Perdew, J. Chem. Phys. 119 (2003) 12129; ibid. 121 (2004) 11507(E).

[181] V. N. Staroverov, G. E. Scuseria, J. Tao, J. P. Perdew, Phys. Rev. B 69 (2004) 075102.

[182] A. D. Becke, M. R. Roussel, Phys. Rev. A 39 (1989) 3761.

[183] A. D. Becke, Int. J. Quantum Chem. 23 (1983) 1915.

[184] A. D. Becke, J. Chem. Phys. 88 (1988) 1053.

[185] A. K. Rajagopal, J. C. Kimball, M. Banerjee, Phys. Rev. B 18 (1978) 2339.

[186] A. D. Becke, Int. J. Quantum Chem. Symp. 28 (1994) 625.

[187] R. Neumann, N. C. Handy, Chem. Phys. Lett. 246 (1995) 381.

[188] A. F. Izmaylov, S. N. Maximoff, G. E. Scuseria (in preparation).

[189] M. Ernzerhof, J. P. Perdew, J. Chem. Phys. 109 (1998) 3313.

[190] L. Constantin, J. P. Perdew (work in progress).

[191] C. Lee, W. Yang, R. G. Parr, Phys. Rev. B 37 (1988) 785.

[192] R. Colle, O. Salvetti, Theor. Chim. Acta 37 (1975) 329.

[193] B. Miehlich, A. Savin, H. Stoll, H. Preuss, Chem. Phys. Lett. 157 (1989) 200.

[194] B. G. Johnson, P. M. W. Gill, J. A. Pople, J. Chem. Phys. 98 (1993) 5612.

[195] J. Tao, P. Gori-Giorgi, J. P. Perdew, R. McWeeny, Phys. Rev. A 63 (2001) 032513.

[196] R. Singh, L. Massa, V. Sahni, Phys. Rev. A 60 (1999) 4135.

[197] C. Filippi, X. Gonze, C. J. Umrigar, in: J. M. Seminario (Ed.), Recent Developments and Applications of Modern Density Functional Theory (Theoretical and Computational Chemistry, Vol. 4), Elsevier, Amsterdam, 1996, p. 295.

[198] Y. Imamura, G. E. Scuseria, R. M. Martin, J. Chem. Phys. 116 (2002) 6458.

[199] R. Colle, O. Salvetti, J. Chem. Phys. 79 (1983) 1404.

[200] Y. Imamura, G. E. Scuseria, J. Chem. Phys. 118 (2003) 2464.

[201] S. F. Boys, N. C. Handy, Proc. Roy. Soc. A 310 (1969) 43.

[202] A. C. Hurley, Electron Correlation in Small Molecules, Academic Press, London, 1976.

[203] T. Tsuneda, K. Hirao, Chem. Phys. Lett. 268 (1997) 510.

[204] T. Tsuneda, T. Suzumura, K. Hirao, J. Chem. Phys. 110 (1999) 10664.

[205] E. I. Proynov, D. R. Salahub, Phys. Rev. B 49 (1994) 7874; ibid. 57 (1998) 12616(E).

[206] E. I. Proynov, A. Vela, D. R. Salahub, Chem. Phys. Lett. 230 (1994) 419; ibid. 234 (1995) 462(E).

[207] E. I. Proynov, E. Ruiz, A. Vela, D. R. Salahub, Int. J. Quantum Chem. Symp. 29 (1995) 61.

[208] E. I. Proynov, S. Sirois, D. R. Salahub, Int. J. Quantum Chem. 64 (1997) 427.

[209] E. Proynov, H. Chermette, D. R. Salahub, J. Chem. Phys. 113 (2000) 10013. 
[210] J. W. Negele, D. Vautherin, Phys. Rev. C 5 (1972) 1472.

[211] R. M. Koehl, G. K. Odom, G. E. Scuseria, Mol. Phys. 87 (1996) 835.

[212] T. Van Voorhis, G. E. Scuseria, Mol. Phys. 92 (1997) 601.

[213] T. Van Voorhis, G. E. Scuseria, J. Chem. Phys. 109 (1998) 400.

[214] S. N. Maximoff, G. E. Scuseria, J. Chem. Phys. 114 (2001) 10591.

[215] A. D. Becke, J. Chem. Phys. 107 (1997) 8554.

[216] A. D. Becke, J. Comput. Chem. 20 (1999) 63.

[217] R. D. Adamson, P. M. W. Gill, J. A. Pople, Chem. Phys. Lett. 284 (1998) 6.

[218] D. J. Tozer, N. C. Handy, J. Chem. Phys. 108 (1998) 2545.

[219] F. A. Hamprecht, A. J. Cohen, D. J. Tozer, N. C. Handy, J. Chem. Phys. 109 (1998) 6264.

[220] G. K.-L. Chan, N. C. Handy, J. Chem. Phys. 112 (2000) 5639.

[221] P. J. Wilson, T. J. Bradley, D. J. Tozer, J. Chem. Phys. 115 (2001) 9233.

[222] H. L. Schmider, A. D. Becke, J. Chem. Phys. 108 (1998) 9624.

[223] H. L. Schmider, A. D. Becke, J. Chem. Phys. 109 (1998) 8188.

[224] A. D. Boese, N. L. Doltsinis, N. C. Handy, M. Sprik, J. Chem. Phys. 112 (2000) 1670.

[225] A. D. Boese, N. C. Handy, J. Chem. Phys. 114 (2001) 5497.

[226] A. D. Boese, N. C. Handy, J. Chem. Phys. 116 (2002) 9559.

[227] C. Y. Lin, M. W. George, P. M. W. Gill, Aust. J. Chem. 57 (2004) 365.

[228] N. C. Handy, A. J. Cohen, Mol. Phys. 99 (2001) 403.

[229] W.-M. Hoe, A. J. Cohen, N. C. Handy, Chem. Phys. Lett. 341 (2001) 319.

[230] A. J. Cohen, N. C. Handy, Chem. Phys. Lett. 316 (2000) 160.

[231] X. Xu, W. A. Goddard, Proc. Natl. Acad. Sci. U.S.A. 101 (2004) 2673.

[232] A. D. Becke, J. Chem. Phys. 98 (1993) 1372.

[233] M. Levy, N. H. March, N. C. Handy, J. Chem. Phys. 104 (1996) 1989.

[234] A. D. Becke, J. Chem. Phys. 98 (1993) 5648.

[235] P. J. Stephens, F. J. Devlin, C. F. Chabalowski, M. J. Frisch, J. Phys. Chem. 98 (1994) 11623.

[236] R. H. Hertwig, W. Koch, Chem. Phys. Lett. 268 (1997) 345.

[237] J. P. Perdew, M. Ernzerhof, K. Burke, J. Chem. Phys. 105 (1996) 9982.

[238] A. Görling, M. Levy, J. Chem. Phys. 106 (1997) 2675.

[239] C. Adamo, V. Barone, J. Chem. Phys. 108 (1998) 664.

[240] M. Ernzerhof, G. E. Scuseria, J. Chem. Phys. 110 (1999) 5029.

[241] C. Adamo, V. Barone, J. Chem. Phys. 110 (1999) 6158.

[242] A. D. Boese, J. M. L. Martin, J. Chem. Phys. 121 (2004) 3405.

[243] A. J. Cohen, N. C. Handy, Mol. Phys. 99 (2001) 607.

[244] J. Baker, P. Pulay, J. Chem. Phys. 117 (2002) 1441.

[245] J. Baker, P. Pulay, J. Comput. Chem. 24 (2003) 1184.

[246] Y. Zhao, J. Z. Pu, B. J. Lynch, D. G. Truhlar, Phys. Chem. Chem. Phys. 6 (2004) 673.

[247] Y. Zhao, B. J. Lynch, D. G. Truhlar, J. Phys. Chem. A 108 (2004) 2715.

[248] Y. Zhao, B. J. Lynch, D. G. Truhlar, J. Phys. Chem. A 108 (2004) 4786.

[249] O. A. Vydrov, G. E. Scuseria, J. Chem. Phys. 121 (2004) 8187.

[250] B. Braïda, P. C. Hiberty, A. Savin, J. Phys. Chem. A 102 (1998) 7872.

[251] M. Grüning, O. V. Gritsenko, S. J. A. van Gisbergen, E. J. Baerends, J. Phys. Chem. A 105 (2001) 9211.

[252] J. P. Dombroski, S. W. Taylor, P. M. W. Gill, J. Phys. Chem. 100 (1996) 6272.

[253] R. D. Adamson, J. P. Dombroski, P. M. W. Gill, Chem. Phys. Lett. 254 (1996) 329.

[254] P. M. W. Gill, R. D. Adamson, Chem. Phys. Lett. 261 (1996) 105.

[255] P. M. W. Gill, Chem. Phys. Lett. 270 (1997) 193. 
[256] R. D. Adamson, P. M. W. Gill, J. Mol. Struct. (Theochem) 398 (1997) 45.

[257] A. Savin, H.-J. Flad, Int. J. Quantum Chem. 56 (1995) 327.

[258] A. Savin, in: J. M. Seminario (Ed.), Recent Developments and Applications of Modern Density Functional Theory (Theoretical and Computational Chemistry, Vol. 4), Elsevier, Amsterdam, 1996, p. 327.

[259] T. Leininger, H. Stoll, H.-J. Werner, A. Savin, Chem. Phys. Lett. 275 (1997) 151.

[260] R. Pollet, A. Savin, T. Leininger, H. Stoll, J. Chem. Phys. 116 (2002) 1250.

[261] P. M. W. Gill, R. D. Adamson, J. A. Pople, Mol. Phys. 88 (1996) 1005.

[262] J. Heyd, G. E. Scuseria, M. Ernzerhof, J. Chem. Phys. 118 (2003) 8207.

[263] J. Heyd, G. E. Scuseria, J. Chem. Phys. 120 (2004) 7274.

[264] J. Heyd, G. E. Scuseria, J. Chem. Phys. 121 (2004) 1187.

[265] H. Iikura, T. Tsuneda, T. Yanai, K. Hirao, J. Chem. Phys. 115 (2001) 3540.

[266] M. Kamiya, T. Tsuneda, K. Hirao, J. Chem. Phys. 117 (2002) 6010.

[267] T. Yanai, D. P. Tew, N. C. Handy, Chem. Phys. Lett. 393 (2004) 51.

[268] Y. Tawada, T. Tsuneda, S. Yanagisawa, T. Yanai, K. Hirao, J. Chem. Phys. 120 (2004) 8425.

[269] A. Szabo, N. S. Ostlund, Modern Quantum Chemistry, Macmillan, New York, 1982.

[270] J. A. Pople, P. M. W. Gill, B. G. Johnson, Chem. Phys. Lett. 199 (1992) 557.

[271] R. Neumann, R. H. Nobes, N. C. Handy, Mol. Phys. 87 (1996) 1.

[272] C. W. Murray, N. C. Handy, G. J. Laming, Mol. Phys. 78 (1993) 997.

[273] P. M. W. Gill, B. G. Johnson, J. A. Pople, Chem. Phys. Lett. 209 (1993) 506.

[274] V. I. Lebedev, Sib. Mat. Zh. 18 (1977) 132.

[275] V. I. Lebedev, A. L. Skorokhodov, Dokl. Akad. Nauk 324 (1992) 519.

[276] V. I. Lebedev, Dokl. Akad. Nauk 338 (1994) 454.

[277] V. I. Lebedev, D. N. Laikov, Dokl. Akad. Nauk 366 (1999) 741.

[278] A. D. Becke, J. Chem. Phys. 88 (1988) 2547.

[279] R. E. Stratmann, G. E. Scuseria, M. J. Frisch, Chem. Phys. Lett. 257 (1996) 213.

[280] I. M. Gelfand, S. V. Fomin, Calculus of Variations, Prentice Hall, Englewood Cliffs, N.J., 1963.

[281] A. V. Arbuznikov, M. Kaupp, V. G. Malkin, R. Reviakine, O. L. Malkina, Phys. Chem. Chem. Phys. 4 (2002) 5467.

[282] A. V. Arbuznikov, M. Kaupp, Chem. Phys. Lett. 381 (2003) 495.

[283] A. V. Arbuznikov, M. Kaupp, Chem. Phys. Lett. 386 (2004) 8.

[284] A. M. Teale, D. J. Tozer, Chem. Phys. Lett. 383 (2004) 109.

[285] W. Hieringer, F. Della Sala, A. Görling, Chem. Phys. Lett. 383 (2004) 115.

[286] L. A. Curtiss, K. Raghavachari, P. C. Redfern, J. A. Pople, J. Chem. Phys. 106 (1997) 1063.

[287] L. A. Curtiss, K. Raghavachari, P. C. Redfern, J. A. Pople, J. Chem. Phys. 112 (2000) 7374.

[288] L. A. Curtiss, P. C. Redfern, K. Raghavachari, J. A. Pople, J. Chem. Phys. 109 (1998) 42.

[289] J. Andzelm, E. Wimmer, J. Chem. Phys. 96 (1992) 1280.

[290] J. Baker, M. Muir, J. Andzelm, J. Chem. Phys. 102 (1995) 2063.

[291] A. C. Scheiner, J. Baker, J. W. Andzelm, J. Comput. Chem. 18 (1997) 775.

[292] S. Kurth, J. P. Perdew, P. Blaha, Int. J. Quantum Chem. 75 (1999) 889.

[293] M. Grüning, O. V. Gritsenko, E. J. Baerends, J. Phys. Chem. A 108 (2004) 4459.

[294] C. A. Coulson, Rev. Mod. Phys. 32 (1960) 170.

[295] A. J. Coleman, V. I. Yukalov, Reduced Density Matrices: Coulson's Challenge, Springer, Berlin, 2000.

[296] P. M. W. Gill, B. G. Johnson, J. A. Pople, M. J. Frisch, Int. J. Quantum Chem. Symp. 26 (1992) 319 . 
[297] P. M. W. Gill, B. G. Johnson, J. A. Pople, M. J. Frisch, Chem. Phys. Lett. 197 (1992) 499.

[298] G. E. Scuseria, J. Chem. Phys. 97 (1992) 7528.

[299] S. Hirata, S. Ivanov, I. Grabowski, R. J. Bartlett, J. Chem. Phys. 116 (2002) 6468.

[300] R. J. Bartlett, I. Grabowski, S. Hirata, S. Ivanov, J. Chem. Phys. 122 (2005) 034104.

[301] E. Engel, A. Höck, R. M. Dreizler, Phys. Rev. A 61 (2001) 032502.

[302] O. V. Gritsenko, E. J. Baerends, Theor. Chem. Acc. 96 (1997) 44.

[303] E. J. Baerends, O. V. Gritsenko, J. Phys. Chem. A 101 (1997) 5383.

[304] P. R. T. Schipper, O. V. Gritsenko, E. J. Baerends, Phys. Rev. A 57 (1998) 1729.

Erratum for the actually published version-Chapter 24 in: Theory and Applications of Computational Chemistry: The First 40 Years (A Volume of Technical and Historical Perspectives), edited by C. E. Dykstra, G. Frenking, K. S. Kim, and G. E. Scuseria, Elsevier, Amsterdam (2005).

p. 675: Eq. (28) should not contain a factor of 1/2. (Pointed out by Oded Hod). 